\title{
A SCUBA imaging survey of ultracompact HII regions
}

\section{The environments of massive star formation ${ }^{\star}$}

\author{
M. A. Thompson ${ }^{1,2}$, J. Hatchell ${ }^{3}$, A. J. Walsh ${ }^{4}$, G. H. Macdonald ${ }^{2}$, and T. J. Millar ${ }^{5,6}$ \\ ${ }^{1}$ Centre for Astrophysics Research, Science \& Technology Research Institute, University of Hertfordshire, College Lane, \\ Hatfield AL10 9AB, UK \\ e-mail: mat@star.herts.ac.uk \\ 2 Centre for Astrophysics \& Planetary Science, School of Physical Sciences, University of Kent, Canterbury CT2 7NR, UK \\ School of Physics, University of Exeter, Stocker Road, Exeter EX4 4QL, UK \\ 4 School of Physics, University of New South Wales, NSW, 2052, Australia \\ 5 Jodrell Bank Centre for Astrophysics, School of Physics and Astronomy, University of Manchester, PO Box 88, Manchester, \\ M60 1QD, UK \\ 6 School of Mathematics and Physics, Queen's University Belfast, Belfast BT7 1NN, UK
}

Received 20 October 2005 / Accepted 28 March 2006

\section{ABSTRACT}

\begin{abstract}
We present a SCUBA submillimetre $(450$ and $850 \mu \mathrm{m})$ survey of the environment of 105 IRAS point sources, selected from the Wood \& Churchwell (1989a) and Kurtz et al. (1994) radio ultracompact (UC) HII region surveys. We detected a total of $155 \mathrm{sub}-\mathrm{mm}$ clumps associated with the IRAS point sources and identified three distinct types of object: ultracompact cm-wave sources that are not associated with any sub-mm emission (sub-mm quiet objects), sub-mm clumps that are associated with ultracompact $\mathrm{cm}$-wave sources (radio-loud clumps); and sub-mm clumps that are not associated with any known ultracompact cm-wave sources (radio-quiet clumps). $90 \%$ of the sample of IRAS point sources were found to be associated with strong sub-mm emission. We consider the sub-mm colours, morphologies and distance-scaled fluxes of the sample of sub-mm clumps and show that the sub-mm quiet objects are unlikely to represent embedded UC HII regions unless they are located at large heliocentric distances. Many of the 2'.5 SCUBA fields contain more than one sub-mm clump, with an average number of companions (the companion clump fraction) of 0.90 . The clumps are more strongly clustered than other candidate HMPOs and the mean clump surface density exhibits a broken power-law distribution with a break at $3 \mathrm{pc}$. We demonstrate that the sub-mm and $\mathrm{cm}$-wave fluxes of the majority of radio-loud clumps are in excellent agreement with the standard model of ultracompact HII regions. We speculate on the nature of the radio-quiet sub-mm clumps and, whilst we do not yet have sufficient data to conclude that they are in a pre-UC HII region phase, we argue that their characteristics are suggestive of such a stage.
\end{abstract}

Key words. stars: formation - ISM: HII regions - ISM: dust, extinction - submillimeter - radio continuum: ISM

\section{Introduction}

Ultracompact (UC) HII regions are perhaps one of the most reliable tracers of recent massive star formation. UC HII regions are defined as dense, compact bubbles of photoionised gas less than $0.1 \mathrm{pc}$ in diameter, surrounding and excited by massive young stellar objects (YSOs). The estimated age of UC HII regions is between $10^{4}-10^{5}$ years, inferred from their spatial diameters and the typical expansion rates of HII regions. Ultracompact HII regions should thus trace the earliest embedded phases of massive star formation, wherein the YSOs have just begun to ionise their surrounding natal molecular clouds. For recent reviews of UC HII regions see Churchwell (2002), Kurtz et al. (2000) or Garay \& Lizano (1999).

Most UC HII regions have to date been identified by their centimetre-wavelength free-free emission, due to the ability of radio-wavelength radiation to penetrate the dense molecular gas and dust cores within which the UC HII regions are embedded. There are two types of radio surveys that have been used to find UC HII regions, each relying on snapshot radio interferometry

* Full Fig. 1, Fig. 2 and Table 2 are only available in electronic form at http://www.edpsciences.org either targeted toward colour-selected IRAS point sources (Wood \& Churchwell 1989a; Kurtz et al. 1994) or over unbiased regions of the Galactic Plane (Becker et al. 1990, 1994; Zoonematkermani et al. 1990; Giveon et al. 2005). The targeted surveys rely upon the Wood \& Churchwell (1989b) IRAS colour selection criteria for UC HII regions, which propose that UC HII regions possess distinctive far-infared colours and can be identified by a discrete region in a $25 / 12 \mu \mathrm{m}$ versus $60 / 12 \mu \mathrm{m}$ colour-colour diagram.

Few UC HII regions have been mapped at a sufficient angular scale or resolution to investigate their dense molecular surroundings and this means that we are relatively ignorant of the location of the UC HIIs within their embedding cores, the global physical properties of the cores and the wider star-forming neighbourhood. Improving our understanding of the enviroments in which massive stars form is crucial to our comprehension of massive star formation and the impact that massive stars have upon their surroundings. In particular it is becoming apparent that a number of UC HII regions are in fact bright compact components of more extended emission (Kurtz et al. 1999; Kim \& Koo 2001). One possible explanation for these extended regions is that the hierarchichal structure of molecular clouds can give rise to 
HII regions with both ultracompact and extended components if the $\mathrm{O}$ star exciting the HII region is displaced from the centre of its embedding core (Kim \& Koo 2001, 2003).

Recently developed millimetre and sub-millimetre wavelength bolometers such as SCUBA (Holland et al. 1999), SHARC (Hunter et al. 1996), MAMBO (Kreysa et al. 1998) and SIMBA (Nyman et al. 2001) have made it possible to rapidly map dust emission from extended regions over arcminute scales at resolutions of 10-20". A number of sub-millimetre and millimetre continuum mapping studies of massive star-forming regions have been carried out (e.g. Hill et al. 2005; Walsh et al. 2003; Mueller et al. 2002; Hatchell et al. 2000; Hunter et al. 2000) but the largest surveys have so far been aimed at $\mathrm{H}_{2} \mathrm{O}$ (Mueller et al. 2002) or $\mathrm{CH}_{3} \mathrm{OH}$ masers (Walsh et al. 2003; Hill et al. 2005) rather than UC HII regions. Although the individual populations of $\mathrm{H}_{2} \mathrm{O}$ masers, $\mathrm{CH}_{3} \mathrm{OH}$ masers and UC HII regions are known to overlap somewhat (e.g. Walsh et al. 1998; Palagi et al. 1993), without a dedicated large-scale UC HII region survey it is difficult to understand the potentially different physical characteristics of masing versus non-masing regions. Nevertheless, surveys of this type are beginning to reveal the density distributions and physical properties of massive starforming cores. Additional dust clumps located near to those containing the active star formation traced by masers or UC HIIs are also being discovered (Hill et al. 2005; Walsh et al. 2003; Hatchell et al. 2000) and the implication is that these clumps may represent earlier evolutionary stages than the maser cores or UC HII regions.

In order to more fully understand the environments of UC HII regions, constrain the physical properties of their embedding dusty clumps and search for nearby associated clumps, we have carried out a sub-mm SCUBA imaging survey of 105 UC HII regions from the Wood \& Churchwell (1989a) and Kurtz et al. (1994) radio catalogues. In this paper, the first of a series reporting our study, we present a description of the survey procedure, the SCUBA images of the UC HII regions and their sub-mm fluxes, plus a statistical analysis of their sub-mm colours, morphologies, clustering properties and likely physical natures. A detailed treatment of the physical properties (e.g. temperature, mass, density profile) of each of the sub-mm sources detected in the survey and their correlation with the presence of hot molecular cores will be presented in subsequent publications.

In Sect. 2 we outline the sample selection, observational and data reduction procedure. The 450 and $850 \mu \mathrm{m}$ images and the fluxes of the sources detected in the survey are presented in Sect. 3. We investigate the sub-mm detection statistics of the UC HII regions and their positional association with sub-mm cores, the morphology of the sub-mm clumps and their clustering properties in Sect. 4. Finally, in Sect. 5 we summarise the initial results of the survey and its implications for the future study of massive star-forming regions.

\section{Observational procedure and data reduction}

\subsection{Sample selection}

The sample of UC HII regions to be observed was drawn from the radio catalogues of Wood \& Churchwell (1989a, hereafter WC89a), and Kurtz et al. (1994, hereafter KCW94). Each of these catalogues consists of a list of compact radio sources observed with the VLA towards IRAS FIR point sources. Although each radio survey consisted of single VLA pointings toward individual IRAS sources their initial survey selection criteria differ somewhat. The WC89a survey selected their target objects from known compact HII regions (Wink et al. 1982; Wood et al. $1988 \mathrm{a}, \mathrm{b})$ that were likely to contain ultracompact components as suggested by their long-wavelength spectra. The KCW94 survey on the other hand selected their targets from a sample of bright IRAS point sources $\left(S_{100 \mu \mathrm{m}}>1000 \mathrm{Jy}\right)$ that solely fulfilled the Wood \& Churchwell (1989b) FIR colour criteria for UC HII regions. KCW94 made no attempt to screen their sample for wellknown objects of other types (e.g. planetary nebulae, compact or extended HII regions) and as will be seen later certain of the KCW94 objects are not true UC HII regions.

The combined WC89a and KCW94 surveys consist of observations of 143 individual IRAS point sources, of which radio emission from UC HIIs was detected toward 101 IRAS point sources. Many of the IRAS sources display multiple radio components: out of 101 IRAS sources with associated radio emission a total of 150 discrete ultracompact radio components are identified in the WC89a and KCW94 surveys. Our initial SCUBA sample was selected from the 143 individual IRAS sources observed by WC89a and KCW94. As the SCUBA "instantaneous" field of view (FOV) is very similar to the primary beam of the VLA at $2 \mathrm{~cm}$ and the IRAS $100 \mu \mathrm{m}$ beam $F W H M$ we chose to observe each IRAS point source with a single SCUBA field.

There are 42 IRAS sources from the WC89a and KCW94 surveys towards which no radio emission was detected at the time of the WC89a and KCW94 surveys. It is possible that these objects may either represent HII regions extended over scales $\geq 10^{\prime \prime}$, which would have been missed by the snapshot interferometric observations of the radio surveys, UC HII regions that were too faint to be detected in the original surveys, or potential massive protostellar objects in an evolutionary phase prior to that of UC HII regions. We searched the SIMBAD database of astronomical catalogues for more recent radio observations of these fields and discovered that ultracompact radio components had been found toward 21 of these IRAS point sources (predominantly from the $5 \mathrm{GHz}$ galactic plane survey of Becker et al. 1994 and Giveon et al. 2005). In compiling this list of ultracompact radio components we matched each survey against the others to determine a unified source list. Where two (or more) surveys report a radio source of similar brightness within $5^{\prime \prime}$ of one another we consolidated these sources as a single detection. Positions and fluxes are used from the survey with the highest signal-to-noise ratio (generally the WC89a or KCW94 surveys). Thus our initial sample comprises 122 IRAS point sources with associated compact centimetre-wave emission ("UC HII regions") and 21 IRAS point sources that are not associated with any detected compact centimetre-wave components. The latter 21 IRAS sources were included in our sample so that we could compare their sub-mm fluxes and morphologies with those dust cores known to contain UC HII regions.

Whilst the initial sample to be observed consisted of 143 IRAS point sources, due to the manner in which our observations were carried out (flexibly scheduled mode, which is described further in Sect. 2.2) it was not possible to complete observations of the entire sample. We were able to map 105 out of the total number of 143 IRAS point sources, of which 94/122 UC HII-associated IRAS point sources and 9/21 non-UC HII-associated point sources were mapped. The mapped fields and their coordinates are given in Table 1 . In terms of the fields containing known ultracompact radio components our survey is thus $\sim 80 \%$ complete. As the selection of the mapped fields from within the larger sample was essentially random (depending upon hour angle, weather conditions, instrument availability 
Table 1. UC HII fields observed during the survey, their central coordinates and estimated distance to the UC HIIs in each field. Fields indicated with a dagger $\left(^{\dagger}\right)$ show no evidence for UC HII regions in the WC89a and KCW94 surveys, or in the literature search described in Sect. 2.1. Fields indicated by an asterisk $\left(^{*}\right)$ were subject to the $450 \mu \mathrm{m}$ calibration problems described in Sect. 2.3. In cases where there is an ambiguity between the near and far kinematic distance both values are listed. Where the distance to the UC HII in the field is not known or there is no known UC HII in the field an ellipsis is given.

\begin{tabular}{|c|c|c|c|c|c|c|c|c|c|}
\hline \multirow{2}{*}{$\begin{array}{l}\text { UC HII } \\
\text { field name }\end{array}$} & \multicolumn{2}{|c|}{ Field centre $(\mathrm{J} 2000)$} & \multirow{2}{*}{$\begin{array}{l}\text { Dist. } \\
(\mathrm{kpc})\end{array}$} & \multirow{2}{*}{$\begin{array}{l}\text { Dist. } \\
\text { Ref. }\end{array}$} & \multirow{2}{*}{$\begin{array}{l}\text { UC HII } \\
\text { field name }\end{array}$} & \multicolumn{2}{|c|}{ Field centre $(\mathrm{J} 2000)$} & \multirow{2}{*}{$\begin{array}{l}\text { Dist. } \\
(\mathrm{kpc})\end{array}$} & \multirow{2}{*}{$\begin{array}{l}\text { Dist. } \\
\text { Ref. }\end{array}$} \\
\hline & RA & Dec & & & & RA & Dec & & \\
\hline G1.13-0.11 & $17: 48: 41.0$ & $-28: 01: 42.4$ & 8.5 & 1 & G31.40-0.26 & $18: 49: 34.7$ & $-01: 29: 08.4$ & 7.3 & 16 \\
\hline $\mathrm{G} 4.41+0.13$ & $17: 55: 17.6$ & $-25: 05: 01.3$ & & & G32.80+0.19 & $18: 50: 30.9$ & $-00: 01: 59.3$ & 12.9 & 17 \\
\hline G5.48-0.24 & $17: 59: 02.7$ & $-24: 20: 54.1$ & 14.3 & 2 & $\mathrm{G} 32.96+0.28^{\dagger}$ & $18: 50: 30.3$ & $+00: 09: 04.7$ & & \\
\hline G5.89-0.39 & $18: 00: 30.4$ & $-24: 04: 00.2$ & 2.0 & 3 & G33.13-0.09 & $18: 52: 09.9$ & $+00: 08: 39.7$ & 7.1 & 7 \\
\hline G5.97-1.17 & $18: 03: 40.5$ & $-24: 22: 44.3$ & 2.7 & 4 & G33.92+0.11 & $18: 52: 50.2$ & $+00: 55: 29.4$ & 7.1 & 5 \\
\hline G6.55-0.10 & 18:00:50.0 & $-23: 20: 32.5$ & 16.7 & 2 & G34.26+0.15 & $18: 53: 18.6$ & $+01: 14: 57.7$ & 4.0 & 4 \\
\hline $\mathrm{G} 8.14+0.23$ & 18:03:00.7 & $-21: 48: 08.4$ & 4.2 & 2 & G35.02+0.35 & $18: 54: 04.2$ & $+02: 01: 33.9$ & 10.0 & 18 \\
\hline G8.67-0.36 & 18:06:19.0 & $-21: 37: 32.3$ & 4.8 & 5 & G35.05-0.52* & $18: 57: 09.0$ & $+01: 39: 03.4$ & 12.7 & 2 \\
\hline $\mathrm{G} 9.62+0.20$ & $18: 06: 13.5$ & $-20: 31: 47.3$ & 5.7 & 6 & $\mathrm{G} 35.57+0.07^{*}$ & $18: 56: 00.7$ & $+02: 22: 52.2$ & 10.2 & 18 \\
\hline G9.88-0.75 & 18:10:17.1 & $-20: 45: 42.5$ & 3.9 & 7 & G35.58-0.03* & $18: 56: 23.5$ & $+02: 20: 37.8$ & 10.1 & 18 \\
\hline G10.10+0.74 & 18:05:13.1 & $-19: 50: 34.7$ & 4.4 & 5 & G37.36-0.23* & 19:00:20.7 & $+03: 50: 15.6$ & & \\
\hline G10.15-0.34 & 18:09:21.0 & $-20: 19: 30.9$ & 6.0 & 2 & G37.55-0.11* & 19:00:16.0 & $+04: 03: 15.1$ & 9.9 & 4 \\
\hline G10.30-0.15 & 18:08:56.2 & $-20: 05: 53.4$ & 6.0 & 2 & G37.77-0.20* & 19:01:00.4 & $+04: 12: 51.4$ & 8.8 & 18 \\
\hline G10.62-0.38 & $18: 10: 28.7$ & $-19: 55: 51.7$ & 4.8 & 5 & G37.87-0.40* & 19:01:53.0 & $+04: 12: 50.1$ & 9.2 & 19 \\
\hline G10.84-2.59 & $18: 19: 11.9$ & $-20: 47: 33.6$ & 1.9 & 7 & G39.25-0.06* & $19: 03: 13.7$ & $+05: 35: 36.9$ & 11.4 & 18 \\
\hline G11.11-0.34 & $18: 11: 33.3$ & $-19: 30: 38.9$ & 5.2 & 7 & $\mathrm{G} 41.13-0.32^{\dagger} *$ & $19: 07: 36.3$ & $+07: 08: 40.3$ & & \\
\hline G11.94-0.62 & 18:14:01.0 & $-18: 53: 25.0$ & 5.2 & 2 & $\mathrm{G} 41.52+0.04^{*}$ & 19:07:03.7 & $+07: 39: 04.1$ & & \\
\hline G12.43-0.05 & $18: 12: 54.6$ & $-18: 11: 08.4$ & 16.7 & 2 & $\mathrm{G} 41.71+0.11^{*}$ & 19:07:09.8 & $+07: 51: 36.1$ & & \\
\hline G12.90-0.25 & $18: 14: 37.3$ & $-17: 52: 02.5$ & 4.0 & 8 & G41.74+0.10* & 19:07:15.5 & $+07: 52: 43.9$ & & \\
\hline G13.19+0.04 & $18: 14: 05.6$ & $-17: 28: 38.7$ & $4.4 / 12.1$ & 9 & G42.42-0.27 & $19: 09: 49.5$ & $+08: 18: 43.8$ & 5.2 & 10 \\
\hline G13.87+0.28 & $18: 14: 35.6$ & $-16: 45: 37.5$ & 4.5 & 10 & G42.90+0.57 & $19: 07: 42.0$ & $+09: 07: 12.4$ & & \\
\hline G15.04-0.68 & $18: 20: 24.8$ & $-16: 11: 35.0$ & 2.1 & 2 & G43.18-0.52 & $19: 12: 08.7$ & $+08: 52: 08.8$ & 4.6 & 2 \\
\hline G18.15-0.28 & $18: 25: 02.3$ & $-13: 15: 50.8$ & 4.2 & 7 & G43.24-0.05 & $19: 10: 35.0$ & $+09: 08: 31.9$ & 11.7 & 18 \\
\hline G18.30-0.39 & $18: 25: 42.3$ & $-13: 10: 19.9$ & 2.9 & 7 & G43.26-0.18 & 19:11:05.9 & $+09: 05: 58.0$ & & \\
\hline G19.07-0.27 & $18: 26: 47.0$ & $-12: 26: 26.5$ & 5.4 & 2 & G43.80-0.13 & $19: 11: 53.3$ & $+09: 35: 46.3$ & 9.0 & 10 \\
\hline G19.49+0.14 & $18: 26: 03.0$ & $-11: 52: 34.4$ & $2.0 / 14.0$ & 9,11 & $\mathrm{G} 43.82+0.39^{\dagger}$ & $19: 10: 03.7$ & $+09: 51: 23.7$ & $\ldots$ & \\
\hline G19.61-0.23 & $18: 27: 38.1$ & $-11: 56: 39.5$ & 4.5 & 2 & $\mathrm{G} 44.26+0.10$ & $19: 11: 56.6$ & $+10: 07: 01.2$ & & \\
\hline G20.08-0.14 & $18: 28: 10.5$ & $-11: 28: 48.6$ & 12.3 & 5 & G45.07+ & $19: 13: 22.1$ & $+10: 50: 53.4$ & 6.0 & 4 \\
\hline G20.99+0.09 & 18:29:04.1 & $-10: 34: 16.2$ & $1.8 / 14.1$ & 12 & G45.12+0.13 & $19: 13: 27.9$ & $+10: 53: 36.7$ & 6.9 & 4 \\
\hline $\mathrm{G} 22.76-0.49^{\dagger}$ & $18: 34: 28.4$ & $-09: 16: 59.7$ & $4.7 / 10.9$ & 12 & $\mathrm{G} 45.20+0.74^{\dagger}$ & $19: 11: 25.0$ & $+11: 14: 37.4$ & & \\
\hline G23.46-0.20 & $18: 34: 44.9$ & $-08: 31: 07.4$ & 9.0 & 2 & G45.40-0.72 & $19: 17: 03.8$ & $+10: 44: 33.8$ & & \\
\hline $\mathrm{G} 23.71+0.17$ & $18: 33: 53.5$ & $-08: 07: 13.8$ & 8.9 & 2 & $\mathrm{G} 45.47+0.05^{*}$ & $19: 14: 25.8$ & $+11: 09: 25.9$ & 6.0 & 2 \\
\hline G23.87-0.12 & $18: 35: 13.4$ & $-08: 06: 52.4$ & $4.6 / 10.9$ & 12 & G69.54-0.98 & 20:10:09.1 & $+31: 31: 34.4$ & 3.0 & 16 \\
\hline G23.96+0.15 & $18: 34: 25.6$ & $-07: 54: 46.8$ & 6.0 & 2 & G70.29+1.60 & 20:01:45.6 & $+33: 32: 44.1$ & 8.3 & 17 \\
\hline $\mathrm{G} 24.39+0.07^{\dagger}$ & $18: 35: 30.6$ & $-07: 33: 44.2$ & & & G76.18+0.13 & $20: 23: 55.7$ & $+37: 38: 10.5$ & 4.4 & 7 \\
\hline $\mathrm{G} 24.47+0.49$ & $18: 34: 09.2$ & $-07: 18: 04.0$ & $5.7 / 9.8$ & 12 & G76.38-0.62 & $20: 27: 26.5$ & $+37: 22: 47.9$ & 1.0 & 7 \\
\hline G24.68-0.16 & 18:36:51.6 & $-07: 24: 52.3$ & $6.3 / 9.1$ & 9 & G77.97-0.01 & $20: 29: 36.4$ & $+39: 01: 17.5$ & 4.4 & 7 \\
\hline G25.38-0.18 & $18: 38: 14.3$ & $-06: 47: 53.3$ & 3.7 & 13 & G78.44+2.66 & $20: 19: 39.3$ & $+40: 56: 30.4$ & 3.3 & 7 \\
\hline G25.65+1.05 & $18: 34: 19.8$ & $-05: 59: 44.2$ & 3.0 & 14 & G79.30+0.28 & $20: 32: 29.3$ & $+40: 16: 05.5$ & 1.0 & 7 \\
\hline $\mathrm{G} 25.72+0.05$ & 18:38:02.8 & $-06: 23: 47.3$ & 14.0 & 2 & G79.32+1.31 & $20: 28: 12.4$ & $+40: 52: 27.7$ & 1.5 & 7 \\
\hline G26.10-0.07 & $18: 39: 11.0$ & $-06: 06: 22.2$ & & & G80.87+0.42 & $20: 36: 52.6$ & $+41: 36: 32.6$ & 2.1 & 7 \\
\hline G26.54+0.42 & $18: 38: 15.6$ & $-05: 29: 37.2$ & $5.2 / 10.0$ & 12 & G106.80+5.31 & $22: 19: 18.2$ & $+63: 18: 46.3$ & 0.9 & 7 \\
\hline $\mathrm{G} 27.28+0.15$ & $18: 40: 35.2$ & $-04: 57: 44.0$ & 15.2 & 2 & G109.87+2.11 & $22: 56: 19.1$ & $+62: 01: 57.4$ & 0.7 & 7 \\
\hline $\mathrm{G} 27.49+0.19$ & $18: 40: 49.0$ & $-04: 45: 15.1$ & $2.5 / 12.6$ & 12 & G110.21+2.63 & $22: 57: 05.2$ & $+62: 37: 44.3$ & 0.7 & 7 \\
\hline G28.20-0.05 & $18: 42: 58.2$ & $-04: 14: 59.8$ & 9.1 & 7 & G111.28-0.66 & $23: 15: 31.5$ & $+61: 07: 09.3$ & 2.5 & 7 \\
\hline G28.29-0.36 & $18: 44: 18.6$ & $-04: 17: 53.1$ & 3.3 & 7 & G111.61+0.37 & $23: 16: 04.8$ & $+60: 02: 59.7$ & 5.2 & 7 \\
\hline G28.60-0.36 & $18: 44: 49.0$ & $-04: 01: 24.9$ & $5.2 / 9.7$ & 12 & G133.95+1.06 & 02:27:01.1 & $+61: 52: 13.7$ & 3.0 & 7 \\
\hline G28.80+0.17* & $18: 43: 16.7$ & $-03: 35: 45.5$ & $6.4 / 8.5$ & 12 & G138.30+1.56 & 03:01:32.3 & $+60: 29: 11.8$ & 3.8 & 7 \\
\hline G28.83-0.23 & $18: 44: 45.4$ & $-03: 45: 18.1$ & $5.1 / 9.8$ & 9 & $\mathrm{G} 188.77+1.07^{*}$ & 06:09:13.8 & $+21: 53: 12.7$ & $\cdots$ & \\
\hline G29.96-0.02 & 18:46:04.1 & $-02: 39: 21.5$ & 7.4 & 5 & $\mathrm{G} 188.79+1.03^{*}$ & 06:09:07.8 & $+21: 50: 38.7$ & 4.1 & 11 \\
\hline G30.54+0.02 & $18: 46: 59.3$ & $-02: 07: 24.5$ & 13.8 & 2 & G188.95+0.92* & 06:08:53.9 & $+21: 38: 36.7$ & 2.2 & 7 \\
\hline G30.78-0.02 & $18: 47: 35.6$ & $-01: 55: 25.9$ & 5.5 & 15 & G213.88-11.84* & $06: 10: 51.0$ & $-06: 11: 54.1$ & 1.0 & 7 \\
\hline $\mathrm{G} 31.28+0.06$ & $18: 48: 12.3$ & $-01: 25: 48.3$ & 7.2 & 7 & & & & & \\
\hline
\end{tabular}

Distance references are: (1) Mehringer et al. (1998); (2) Wood \& Churchwell (1989a); (3) Acord et al. (1998); (4) Churchwell et al. (1990); (5) Fish et al. (2003); (6) Hofner et al. (1994); (7) Kurtz et al. (1994); (8) van der Tak et al. (2000); (9) kinematic distance evaluated from $\mathrm{CH}_{3} \mathrm{OH}_{\text {maser }}$ velocity given by Szymczak et al. (2000); (10) Harju et al. (1998); (11) kinematic distance evaluated from $\mathrm{CH}_{3} \mathrm{OH}$ maser velocity given by Caswell et al. (1995); (12) kinematic distance evaluated from Hydrogen radio recombination line velocity given by Kuchar \& Clarke (1997); (13) Blum et al. (2000); (14) Zavagno et al. (2002); (15) Motte et al. (2003); (16) Palagi et al. (1993); (17) Araya et al. (2002); (18) Watson et al. (2003); (19) Codella et al. (1994). 
etc.) we are confident that there is little selection bias within our final list of UC HII regions.

\subsection{Observational procedure}

Our observations were carried out using the SCUBA (Holland et al. 1999) common-user bolometer array in operation at the James Clerk Maxwell Telescope $\left(\mathrm{JCMT}^{1}\right)$. The observations were performed in a flexibly-scheduled mode whereby observations are not scheduled over a pre-defined period but are carried out over a 6-month observing semester according to the appropriate weather (atmospheric opacity) band, the visibility of the sources from the telescope and the scientific priority of the observations. The survey data were thus obtained by visiting observers over several separate periods across the 98B (Aug.-Jan.) and 99A (Feb.-July) observing semesters at the JCMT.

SCUBA is comprised of two bolometer arrays, a short-wave array of 91 pixels optimised for operation at $450 \mu \mathrm{m}$ and a longwave array of 37 pixels optimised for operation at $850 \mu \mathrm{m}$. Both arrays simultaneously sample a similar field of view (approx $2^{\prime}$ square), although the spacing between individual bolometers on the array means that not all of the field of view is sampled instantaneously. To fill in the gaps in spatial coverage the telescope secondary mirror is moved in a 64-point pattern ("jiggling"), whilst also chopping at a frequency of $1 \mathrm{~Hz}$ to remove the sky emission. This procedure is commonly known as a "jiggle-map" and provides maps with full spatial sampling at both wavelengths.

As the SCUBA FOV is approximately the same size as the VLA primary beam at $2 \mathrm{~cm}$ and the IRAS $100 \mu \mathrm{m}$ beam area we obtained a single jiggle-map centred at the coordinates of either the UC HII region radio emission or the position of the IRAS source (where no radio information was available). For the UC HII regions contained in the KCW94 survey we mistakenly used the IRAS positions rather than the radio positions, which resulted in the array being slightly offset from the main sub-mm emission. However, the SCUBA FOV was found to be larger than the positional offsets introduced and thus there is little effect upon the data in question. Additional maps with positional offsets were obtained for a number of sources where the mapped emission was affected by noisy bolometers or by being located at the edge of the FOV.

Each source was integrated on for three of the 64-point SCUBA jiggle cycles (also known as SCUBA "integrations") for a total on-source integration time of $192 \mathrm{~s}$. This typically resulted in 1- $\sigma$ rms noises of $\sim 1.5$ Jy per $8^{\prime \prime} F W H M$ beam at $450 \mu \mathrm{m}$ and $\sim 0.1$ Jy per $14^{\prime \prime} F W H M$ beam at $850 \mu \mathrm{m}$. All sources were observed with a chop throw of $120^{\prime \prime}$ in azimuth. As SCUBA is located at the Nasmyth focus of the JCMT and does not possess a beam rotator, this means that the chop positions are also a function of elevation.

Regular pointing checks were made and the average pointing offset was found to be $\leq 5^{\prime \prime}$. Hourly skydips were carried out at approximately the same azimuth as the observations to estimate the atmospheric zenith optical depth at at 450 and $850 \mu \mathrm{m}$. Values derived from the skydips were compared to and found consistent with those extrapolated from the fixed-azimuth measurements at $225 \mathrm{GHz}$ made every $10 \mathrm{~min}$ by the CSO tipping radiometer. Absolute flux calibration and beam maps of the

1 The JCMT is operated by the Joint Astronomy Centre on behalf of PPARC for the UK, The Netherlands Organisation of Scientific Research, and the National Research Council of Canada. primary flux calibrator Uranus were also obtained at least once per observing session.

\subsection{Data reduction}

The data were reduced using a combination of the automated SCUBA reduction pipeline ORACDR (Economou et al. 2002), the SCUBA reduction package SURF (Jenness \& Lightfoot 2000) and the Starlink image analysis package KAPPA (Currie $\&$ Bell 2002). The reduction procedure for 450 and $850 \mu \mathrm{m}$ data was essentially the same and proceeded along the following outline. Initially the chopping and nodding positions were subtracted from the on-source data to form a time-ordered series of sky-subtracted bolometer measurements. Given that the UC HIIs are likely to be embedded in larger giant molecular cloud complexes, it is likely that extended emission from these regions is present in the chopping positions and is subtracted from the onsource sub-mm fluxes by this procedure. The fluxes quoted in this paper should thus be strictly regarded as lower limits to the true sub-mm fluxes.

The time-ordered bolometer data were then corrected for atmospheric extinction using an optical depth value interpolated from skydips carried out before and after the jiggle-map. At this stage bolometers with a mean noise in excess of $100 \mathrm{nV}$ were blanked and transient bolometer noise spikes were removed by applying a $5 \sigma$ clip to the data. As several of the sources were found to be strongly centrally-peaked, care was taken to avoid the removal of the central pixel by this despiking procedure. Residual sky variations between individual bolometers were removed by specifying emission-free bolometers and using the SURF task remsky. The time-ordered data were then regridded to J2000 sky coordinates with the SURF task rebin.

Absolute flux calibration was carried out using the calibration maps of Uranus. Predicted fluxes for Uranus were estimated using the values given by the Starlink package FLUXES (Privett et al. 1998) and on the JCMT calibrator webpage respectively. Flux correction factors (FCFs) for each wavelength were then determined by dividing the predicted flux by the measured peak value of the calibrator. By monitoring the variation in FCF over each observing period it was found that the absolute flux calibration was accurate to $30 \%$ at $450 \mu \mathrm{m}$ and $10 \%$ at $850 \mu \mathrm{m}$. These errors in calibration are predominantly due to variations of the line-of-sight optical depth on timescales smaller than can be accounted for by skydips. Each jiggle-map was calibrated in units of Jy/beam by multiplying by the appropriate FCF. The FWHMs and peak values of the telescope main and error beams were determined by fitting two Gaussians to azimuthal averages of the maps of the primary calibrator (Uranus).

A number of images, mainly taken on a single night, were found to be essentially uncalibratable at $450 \mu \mathrm{m}$ due to either large values of or rapid variations in the $450 \mu \mathrm{m}$ atmospheric optical depth. Although there is a close linear relation between the 450 and $850 \mu \mathrm{m}$ zenith optical depth (Archibald et al. 2002), the atmosphere is typically more unstable and much more optically thick (by a factor of 4 or more) at $450 \mu \mathrm{m}$. The result of this is that whilst the $450 \mu \mathrm{m}$ data was unusable for the affected periods, the $850 \mu \mathrm{m}$ data was relatively unaffected. We do not present the affected $450 \mu \mathrm{m}$ data from these periods, which is a total of 18 images.

The calibrated images were then converted into FITS format and deconvolved to remove the contribution from the error beam. The deconvolution was performed using the clean task in MIRIAD (Sault et al. 1995) with a circularly symmetric twocomponent Gaussian beam derived from azimuthal averages of 
the primary calibrator maps in a manner similar to that described in Hogerheijde \& Sandell (2000). Each image was cleaned down to a cutoff level of twice the $1 \sigma$ rms noise and then restored back to a resolution appropriate for the wavelength ( 8 " for $450 \mu \mathrm{m}$ and $14^{\prime \prime}$ for $\left.850 \mu \mathrm{m}\right)$. The advantage of this technique is that the different error beam contributions from each wavelength are removed, facilitating comparison of $450 \mu \mathrm{m}$ and $850 \mu \mathrm{m}$ maps and allowing the integrated fluxes to be determined more accurately.

After deconvolution to remove the error beam the $450 \mu \mathrm{m}$ images were inspected and those displaying insufficient signalto-noise to permit accurate source identification were smoothed from a native resolution of $8^{\prime \prime}$ to the same resolution as that in the $850 \mu \mathrm{m}$ images $\left(14^{\prime \prime}\right)$. Sacrificing the angular resolution of the $450 \mu \mathrm{m}$ images results in an improvement of the signal-tonoise ratio for extended emission by approximately a factor of 3 , which considerably aided in the identification of faint $450 \mu \mathrm{m}$ sources.

\section{Results}

\subsection{Deconvolved images}

We present the final deconvolved SCUBA images in Figs. 1 and 2, marked with the positions of the UC HII regions in each image. Figure 1 contains images of sources that were detected at 450 and $850 \mu \mathrm{m}$, whereas Fig. 2 contains images of those sources with data only at $850 \mu \mathrm{m}$, either because the $450 \mu \mathrm{m}$ emission was undetected or due to the $450 \mu \mathrm{m}$ calibration problems discussed in the previous section. Only those SCUBA fields with detected sub-mm sources are included in the figures. Submm emission was detected toward $\sim 90 \%$ of the sample (92 out of 105 fields). Here we outline a few attributes of the SCUBA images in order to aid in their interpretation.

"Holes" in the $850 \mu \mathrm{m}$ images result from the blanking of noisy bolometers in the data reduction process. Typically between 1 and 3 bolometers were blanked from each $850 \mu \mathrm{m}$ image and any flux from sources found near the blanked image regions may be underestimated. Individual sources whose fluxes may be affected are indicated in Table 2 . Noisy bolometers were also blanked from the $450 \mu \mathrm{m}$ data, but as the short wavelength array in SCUBA has a higher spatial sampling, the blanking of bolometers from the short-wavelength array does not cause gaps in the spatial coverage of the array. Negative "bowls" of emission are also present in some images (e.g. G13.19+0.04 in panel $73 \mathrm{~L}^{2}$, and $\mathrm{G} 25.38-0.18$ in panel $75 \mathrm{~L}$ ) and these bowls are caused by the presence of sub-mm emission in the chopping positions (i.e. approximately $120^{\prime \prime}$ in azimuth from the field centre). The morphology of a number of these bowls indicates that the emission is compact and resembles that of the positive emission features seen in the sub-mm image. These compact negative bowls may indicate the presence of other sub-mm cores located near the chopping positions, possibly similar to those associated with the UC HII regions in the main image.

The flux contour levels on each image were determined by a dynamic range power-law fitting scheme in order to emphasise both the low-level and bright emission. Logarithmic contour levels were fitted to the dynamic range of the image $D$ (defined as the image peak divided by the $1 \sigma \mathrm{rms}$ noise) following the relation $D=3 \times N^{i}+3$, where $N$ is the number of contours (in this case 9) and $i$ is the contour power-law index. The minimum

2 Note that the panel numbers refer to individual images in Figs. 1 and $2 . \mathrm{L}$ and $\mathrm{S}$ refer to the long $(850 \mu \mathrm{m})$ and short $(450 \mu \mathrm{m})$ wavelength channels of SCUBA. power-law index used was one, which results in linear contours starting at a level of $3 \sigma$ and spaced by $3 \sigma$. This dynamic powerlaw contouring scheme was found to give excellent results in both high and low dynamic range images, concentrating the contours around low surface brightness features in the images to adequately represent low-level structure in the images.

The SCUBA images show that the sub-mm emission associated with UC HII regions follows a range of morphologies: from single, bright, centrally peaked compact cores (e.g. G20.08-0.14 in panel $22 \mathrm{~L}, \mathrm{G} 35.02+0.35$ in panel $46 \mathrm{~L}$ ); to more complex multiply-peaked regions with peaks both associated and unassociated with known UC HII regions (e.g. G28.29-0.36 in panel 36L); and ridge-shaped structures with multiple condensations strung along the ridge (e.g. G27.28+0.15 in panel 33L, $\mathrm{G} 79.30+0.28$ in panel 59L). Often the sub-mm cores that are not associated with the UC HII regions in an image are of comparable brightness or sometimes even brighter than the neighbouring cores that are (e.g. G8.14+0.23 in panel 4L, G25.72+0.05 in panel $31 \mathrm{~L})$. The SCUBA images of the non-UC HII associated IRAS point sources (those indicated by $\mathrm{a}^{\dagger}$ in Table 1 ) also reveal the presence of bright radio-quiet sub-mm cores similar in characteristics and appearance to the cores that are known to be associated with UC HII regions (e.g. G13.19+0.04 in panel 73L). If these radio-quiet sub-mm cores are forming massive stars in a similar manner to their radio-loud UC HII-associated neighbours, it is possible that the radio-quiet cores represent an earlier evolutionary phase of star formation prior to the development of an UC HII region as suggested by Garay et al. (2004). We will dwell further on this hypothesis in Sect. 4.

\subsection{Source identification}

Individual sources within the 450 and $850 \mu \mathrm{m}$ images were identified using the source extraction algorithm SEXTRACTOR (Bertin \& Arnouts 1996). Whilst SEXTRACTOR was initially developed as a source extraction routine for visible and infraredwavelength images, it nevertheless provides the means of separating the often closely blended sub-mm cores in the SCUBA images and measuring their individual fluxes in a consistent fashion. As SEXTRACTOR treats each pixel of the target image in a statistically independent way, whereas each of our sub$\mathrm{mm}$ images is gridded in pixels smaller than the beam FWHM (and are thus not statistically independent), it was necessary to account for the beam area in the final measured fluxes and their uncertainties. We ran SEXTRACTOR on each of our images, setting the analysis threshold to be three times the rms noise in each image. The rms noise in each image was determined by hand from the standard deviation of source-free pixels, as it was found that SEXTRACTOR could not make an accurate automatic determination of this quantity in the relatively small and crowded SCUBA images (as compared to typical visiblewavelength data).

A combined source catalogue was compiled from the individual SEXTRACTOR runs on each SCUBA image. Beamcorrected 450 and $850 \mu \mathrm{m}$ fluxes were determined for each source in the catalogue (using the FLUX_AUTO keyword) and were checked against a random sample of fluxes determined by manual aperture photometry. In all cases the fluxes determined by SEXTRACTOR were consistent with those measured by manual aperture photometry. We also compared the measured fluxes of those sources from our catalogue that have been published in the literature. We share 11 fields with the methanol maser survey of Walsh et al. (2003) and in all but two cases the integrated and peak fluxes are consistent to within the 

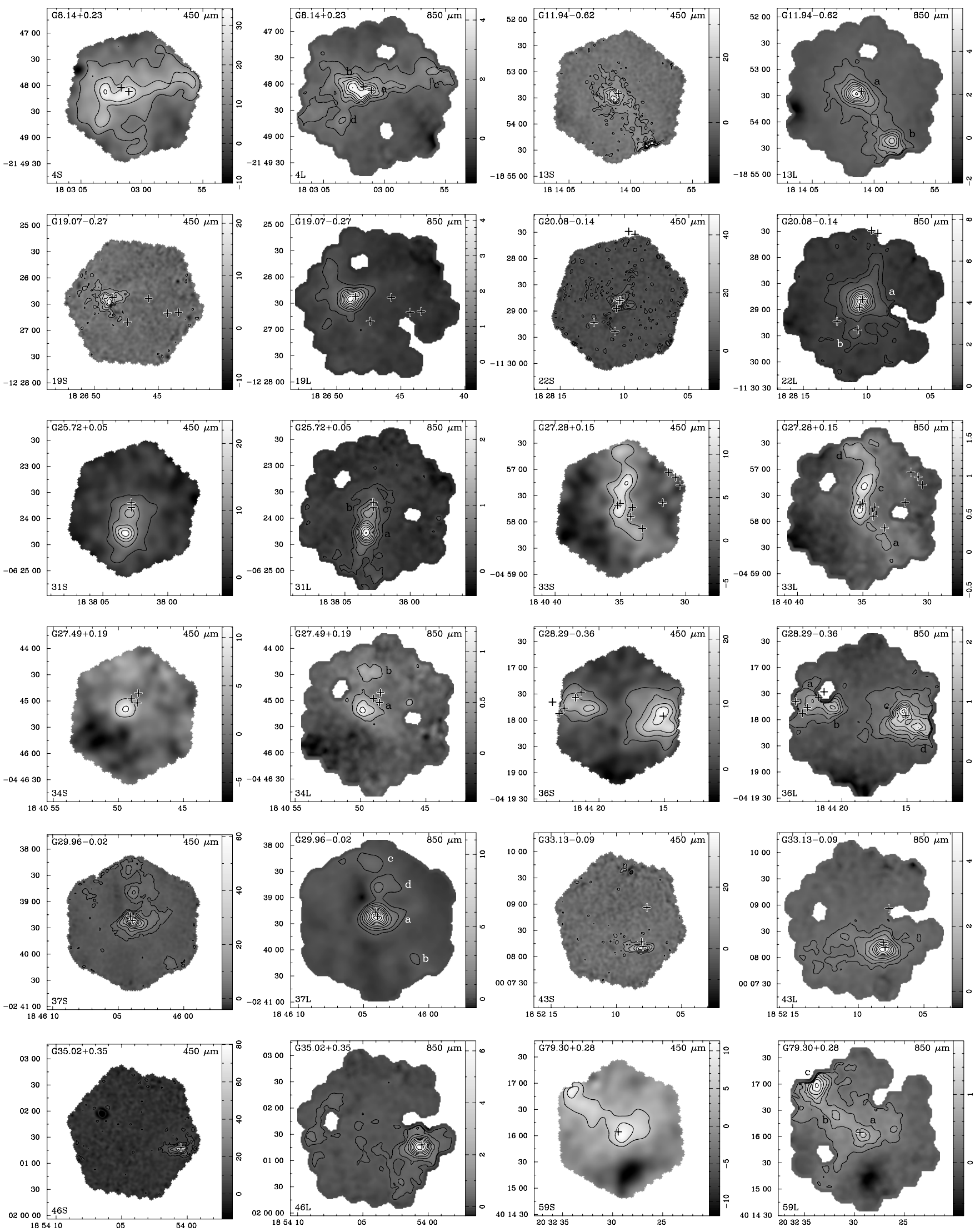

Fig. 1. SCUBA images from the survey with detections at both 450 and $850 \mu \mathrm{m}$. The images displayed here are those discussed further in the text of the paper. Each UC HII region is represented by a pair of images at $450 \mu \mathrm{m}$ (left image) and $850 \mu \mathrm{m}$ (right image). Coordinates are Right Ascension and Declination in the J2000 system. Crosses indicate the positions of ultracompact HII regions from Wood \& Churchwell (1989a), Kurtz et al. (1994), Becker et al. (1994) or Giveon et al. (2005). All images have been deconvolved with a model of the JCMT beam to remove the contribution from the error lobe and $450 \mu \mathrm{m}$ images with limited signal-to-noise have been smoothed to the same resolution as the $850 \mu \mathrm{m}$ images to improve the source detections. The full version of this figure is available in electronic form. 
Table 3. SCUBA fields from the survey with no detected sub-mm emission (to a level of $3 \sigma$ ). Fields indicated with a dagger $\left(^{\dagger}\right)$ show no evidence for UC HII regions in the WC89a and KCW94 surveys, or in the literature search described in Sect. 2.1.

\begin{tabular}{llc}
\hline \hline $\begin{array}{l}\text { UC HII } \\
\text { field name }\end{array}$ & $\begin{array}{l}\text { IRAS PSC } \\
\text { identifier }\end{array}$ & $\begin{array}{c}850 \mu \mathrm{m} \text { flux upper limit } \\
(\mathrm{Jy} / \text { beam })\end{array}$ \\
\hline G24.39+0.07 & $18328-0735$ & 0.3 \\
G26.10-0.07 & $18365-0609$ & 0.4 \\
G32.96+0.28 & $18481-0014$ & 0.2 \\
G37.36-0.23 & $18578+0345$ & 0.4 \\
G41.13-0.32 & $19051+0704$ & 0.2 \\
G41.52+0.04 & $19046+0734$ & 0.2 \\
G41.71+0.11 & $19048+0748$ & 0.2 \\
G42.90+0.57 & $19054+0901$ & 0.1 \\
G43.26-0.18 & $19087+0900$ & 0.1 \\
G43.82+0.39 & $19077+0946$ & 0.2 \\
G44.26+0.10 & $19096+1001$ & 0.1 \\
G45.20+0.74 & $19090+1109$ & 0.2 \\
G45.40-0.72 & $19147+1040$ & 0.3 \\
G188.77+1.07 & $06061+2151$ & 0.2 \\
\hline
\end{tabular}

uncertainties. An inspection of the two images (G15.04-0.68 and G28.29-0.36) where the flux measurements are inconsistent reveals that the likely cause is due to the confused nature of these regions and the different chop positions used by ourselves and Walsh et al. (2003). Our measured fluxes of the sub-mm cores associated with G76.38-0.62, G25.65+1.05 and G106.80+5.31 (also known as S 106, RAFGL 7009S and S 140 respectively) also agree very closely with the sub-mm measurements of Richer et al. (1993), McCutcheon et al. (1995) and Minchin et al. (1995), taking into account the slightly different filter bandwidths used in these observations.

The combined source catalogue with the centroid position of each sub-mm source, its peak flux and integrated flux at both 450 and $850 \mu \mathrm{m}$ are given in Table 2 . The positions quoted for each source are usually taken from the $850 \mu \mathrm{m}$ images (unless otherwise stated) as the the $850 \mu \mathrm{m}$ images have a considerably higher signal-to-noise ratio. Where it was possible to deblend sources at $450 \mu \mathrm{m}$ but not at $850 \mu \mathrm{m}$ images the centroid positions are those measured from the $450 \mu \mathrm{m}$ images. The errors quoted in Table 2 include the absolute calibration errors of $30 \%$ at $450 \mu \mathrm{m}$ and $10 \%$ at $850 \mu \mathrm{m}$ combined in quadrature with the intrinsic measurement error derived from the image rms noise.

Out of the total 105 SCUBA fields observed as part of the survey we have identified 155 sub-mm sources at either 450 or $850 \mu \mathrm{m}$. Approximately $10 \%$ of the fields observed (13 in total) did not display any sub-mm emission down to a level of $3 \sigma$. Approximately half of the non-detected SCUBA fields $(7 / 13)$ are associated with ultracompact centimetre-wave radio components. As these objects do not display significant sub-mm emission, which indicates a low column density of dust, it is unlikely that the centimetre-wave radio emission traces embedded UC HII regions unless the distance to these objects is large. We will discuss these objects in more detail in Sect. 4. The non-detected SCUBA fields and their upper $850 \mu \mathrm{m}$ flux limits are are indicated in Table 3.

\subsection{Notes on individual sources}

In this section we describe selected fields from the survey in further detail to aid in the interpretation of their sub-mm emission. We briefly describe their morphology in the sub-mm, their associated UC HII regions (or lack of them) and whether the fields are better known by another name than their galactic coordinates.

G1.13-0.11: panels $1 \mathrm{~S}$ and $1 \mathrm{~L}$ This source is also known as Sgr D (Mehringer et al. 1998) and is a cluster of compact HII regions mostly less than $10^{\prime \prime}$ in diameter. The cluster is surrounded by a low-intensity extended cm-wave continuum component and is dominated by a single compact source that is consistent with excitation by an O5.5 star (Mehringer et al. 1998). We identify one sub-mm clump associated with G1.13-0.11, which takes the form of an extended ridge running roughly NE-SW across the SCUBA FOV. This morphology is consistent with the $350 \mu \mathrm{m}$ map of this source obtained by Hunter et al. (2000). The central region of the ridge possesses two small peaks which may indicate the presence of more than one sub-mm clump, however we are unable to separate the emission into two discrete cores. The dominating compact HII region is located close to the central peak, suggesting that it is embedded within the dust ridge. The presence of $\mathrm{H}_{2} \mathrm{CO}$ absorption lines at the molecular cloud velocity towards this compact HII region strengthens this hypothesis (Mehringer et al. 1998). The large-scale radio and sub-mm morphology of this region suggest that the extended $\mathrm{cm}$-wave emission represents a blister HII region. Because G1.13-0.11 is located so near to the Galactic Centre on the sky it is difficult to estimate an accurate distance. Following Mehringer et al. (1998) we assume that the distance to G1.13-0.11 is the same as that to the Galactic Centre $(8.5 \mathrm{kpc})$, although we caution that the true distance is likely to be greater than this estimate (see Mehringer et al. 1998 for a more detailed argument).

G5.89-0.39: panels 2S and 2L We identify one sub-mm core in this region (G5.887-0.394SMM) which is also the wellknown outflow source OH 5.89-0.39 (Zijlstra et al. 1990) or W28 A2 (Harvey \& Forveille 1988). G5.89-0.39 is a wellknown luminous massive star-forming region, displaying compact cm-wave emission from an UC HII region (WC89a), $\mathrm{H}_{2} \mathrm{O}$, $\mathrm{OH}$ and $\mathrm{CH}_{3} \mathrm{OH}$ maser emission (Hofner \& Churchwell 1996; Palagi et al. 1993; Argon et al. 2000; Val'tts et al. 2000) and thermal emission from a variety of molecular tracers (e.g. Fontani et al. 2002; Thompson et al. 1999; Gomez et al. 1991). G5.89-0.39 has also been observed comprehensively in the infrared and millimetre-wave continuum (Feldt et al. 1999) and in $350 \mu \mathrm{m}$ continuum emission (Hunter et al. 2000; Mueller et al. (2002). The morphologies of the millimetre and $350 \mu \mathrm{m}$ maps agree well with our 450 and $850 \mu \mathrm{m}$ maps. The sub$\mathrm{mm}$ core appears to possess a clumpy substructure, with the UC HII region slightly offset from the peak of the sub-mm emission. This may indicate that the sub-mm core contains multiple sub-components, which is consistent with the scenario proposed by Feldt et al. (1999). For further discussion of the structure of the sub-mm clump associated with G5.89-0.39 the reader is referred to the models of Hatchell \& van der Tak (2003) and Feldt et al. (1999).

G5.97-1.17: panels 3S and 3L This object is also the wellknown Hourglass region of the Lagoon Nebula and is home to the $\mathrm{O} 7$ star Herschel 36. The Lagoon Nebula has been extensively investigated by Tothill et al. (2002) and the interested reader is referred to this paper and its companion (White et al. 1997) for an overview of the star formation within the Lagoon Nebula. We identify two sub-mm clumps in our SCUBA images: G5.972-1.159SMM (which corresponds to clump WC1 
in Tothill et al. 2002) and G5.974-1.175SMM (clump HG in Tothill et al. 2002). We note that the sub-mm fluxes measured in this paper are roughly a factor of 2 greater than those measured by Tothill et al. (2002). This is more than likely due to the different procedures used to measure the sub-mm fluxes: Tothill et al. use a Gaussian fitting procedure to extract the clumps from local background emission, whereas our aperture photometry is likely to include this extended component.

G6.55-0.10: panel 70L This object is also known as W28 A1 and exhibits maser emission from both $\mathrm{H}_{2} \mathrm{O}$ and $\mathrm{CH}_{3} \mathrm{OH}$ masers (Churchwell et al. 1990; Walsh et al. 1998), plus thermal $\mathrm{NH}_{3}$ emission (Churchwell et al. 1990). Kim \& Koo (2001) show that the WC89a UC HII region represents the peak of a compact HII region approximately $30^{\prime \prime}$ in diameter. The sub-mm images reveal a single diffuse core in the centre of the field, which is positionally associated with both the compact HII region and the $\mathrm{H}_{2} \mathrm{O}$ and $\mathrm{CH}_{3} \mathrm{OH}$ masers.

G8.14+0.23: panels 4S and 4L The sub-mm emission in this fields follows a ridge-like morphology, with four identified sub-mm clumps (G8.143+0.219SMM, G8.140+0.224SMM, G8.135+0.245SMM, G8.136+0.212SMM). This object has also been mapped by Walsh et al. (2003) and our 450 and $850 \mu \mathrm{m}$ images agree closely with those of Walsh et al. Kim \& Koo (2003) present wide-field maps of G8.14+0.23 in the ${ }^{13} \mathrm{CO} J=1-0$ line, which show a ridge of molecular emission in close correspondence with the ridge seen in our SCUBA images. The star-formation activity in this region appears restricted to the clump G8.140+0.224SMM, which is positionally associated with two UC HII regions (WC89a; Becker et al. 1994) and an $\mathrm{H}_{2} \mathrm{O}$ maser (Palagi et al. 1993). There is also a $\mathrm{CH}_{3} \mathrm{OH}$ maser detected toward this region (Walsh et al. 1998), which is located at the Western edge of clump G8.143+0.219SMM.

G8.67-0.36: panels 5S and 5L There are two sub-mm clumps identified in the SCUBA images (G8.683-0.368SMM and G8.671-0.357SMM). Both clumps are associated with $\mathrm{CH}_{3} \mathrm{OH}$ masers (Caswell et al. 1995), whereas only the brightest submm clump G8.671-0.357SMM is associated with an $\mathrm{H}_{2} \mathrm{O}$ maser (Palagi et al. 1993) and an UC HII region (WC89a). Mueller et al. (2002) detected only a single clump in their $350 \mu \mathrm{m}$ map of G8.67-0.36, as the edge of their mapped field truncates G8.683-0.368SMM.

G9.62+0.20: panels $6 \mathrm{~S}$ and $6 \mathrm{~L}$ This object is the well studied massive star-forming region $\mathrm{G} 9.62+0.20$, which is host to several UC HII regions, $\mathrm{CH}_{3} \mathrm{OH}$ and $\mathrm{H}_{2} \mathrm{O}$ masers and a hot molecular core (Persi et al. 2003; Testi et al. 2000; Hofner et al. 1996). We detect a single sub-mm clump in this field that appears to be elongated along the NW-SE axis, in agreement with the SCUBA map of Walsh et al. (2003) and the $350 \mu \mathrm{m}$ maps of Hunter et al. (2000) and Mueller et al. (2002). For a wider overview of the star-formation in this region the reader is directed to the excellent summaries by Hofner et al. (1996) and Persi et al. (2003).

G10.15-0.34/G10.30-0.15: panels 8S and 8L and 9S and 9L These two complexes of UC HII regions identified by WC89a lie in the W31 Giant Molecular Cloud. Kim \& Koo (2001) present wide-field $20 \mathrm{~cm}$ maps of both these regions which reveal that the WC89a UC HII regions are associated with extended radio emission (a shell morphology in the case of G10.15-0.34 and a bipolar HII region morphology for G10.30-0.15). The sub$\mathrm{mm}$ ridge observed in G10.30-0.15 aligns well with the centre of the bipolar HII region observed by Kim \& Koo (2001) and the elongated CS emssion mapped by Kim \& Koo (2002). This suggests that the stars exciting the HII region are still embedded in a dense flattened molecular clump seen edge-on, but their UV photons are escaping from the top and bottom of the clump to ionise the surrounding material (as in, for example, S 106; Richer et al. 1993). G10.30-0.15 is also associated with $\mathrm{CH}_{3} \mathrm{OH}$ maser emission (Walsh et al. 1998; Goedhart et al. 2000).

G10.84-2.59: panels 11S and 11L This source is also known as GGD 27, which comprises an infrared reflection nebula (Yamashita et al. 1987), a cluster of potentially massive YSOs (Aspin \& Geballe 1992; Bica et al. 2003), the HH 80-81 thermal radio jet (Marti et al. 1993) an $\mathrm{H}_{2} \mathrm{O}$ maser (Gómez et al. 1995) and a $\mathrm{CH}_{3} \mathrm{OH}$ maser (Val'tts et al. 2000). Gómez et al. (2003) present high resolution $\mathrm{NH}_{3}$ and $\mathrm{HCN}$ images of the GGD 27 region, showing that the peak of the molecular emission is closely associated with the $\mathrm{H}_{2} \mathrm{O}$ maser. Although at the limit of our SCUBA positional accuracy, we note that the sub-mm peak identified in this field (G10.842-2.594SMM) is displaced by $\sim 6^{\prime \prime}$ from that of the $\mathrm{H}_{2} \mathrm{O}$ maser. It is likely that the UC HII region identified by KCW94 is actually the central source of the $\mathrm{HH}$ 80-81 thermal jet. The luminosity of the UC HII region suggests that a B0-B1 star is responsible for its excitation (KCW94).

G11.94-0.62: panels $13 \mathrm{~S}$ and 13L Two sub-mm sources are identified in this field (G11.936-0.618SMM, G11.918$0.615 \mathrm{SMM})$. The southernmost source (G11.918-0.615SMM) is associated with an $\mathrm{H}_{2} \mathrm{O}$ maser (Hofner \& Churchwell 1996) whereas the brighter central source (G11.936-0.618SMM) is associated with an UC HII region (WC89a), $\mathrm{H}_{2} \mathrm{O}$ (Hofner \& Churchwell 1996) and $\mathrm{CH}_{3} \mathrm{OH}$ masers (Val'tts et al. 2000). No cm-wave radio emission is found to be associated with the southernmost sub-mm clump G11.918-0.615SMM. Given the presence of an $\mathrm{H}_{2} \mathrm{O}$ maser in this core the lack of cm-wave radio continuum emission suggests that G11.918-0.615SMM may be at an earlier phase of massive star formation than G11.936-0.618SMM. These two sub-mm sources were also observed by Walsh et al. (2003).

G12.90-0.25: panels 14S and 14L This source is the wellstudied star-forming region W33A (e.g. Jaffe et al. 1984). W33A is a deeply embedded luminous infrared star-forming region exhibiting several molecular absorption lines (Grim et al. 1991; Palumbo et al. 1995). We have identified two submm clumps within our SCUBA images of which the brightest (G12.908-0.261SMM) is associated with W33A. Numerous masers are associated with this sub-mm clump; OH (Argon et al. 2000), $\mathrm{H}_{2} \mathrm{O}$ (Braz \& Epchtein 1983) and $\mathrm{CH}_{3} \mathrm{OH}$ (Szymczak et al. 2000; Caswell et al. 1995). Van der Tak et al. (2000) have also mapped the W33A clump in millimetre-wave continuum at high angular resolution, which reveals two embedded compact millimetre cores. One of these cores (W33A MM 1) is associated with a weak centimetre continuum source that is consistent with an ionised stellar wind (Rengarajan \& Ho 1996). In contrast the remaining sub-mm clump in this field (G12.908-0.261SMM) displays no known cm-wave continuum or maser emission. This clump was also undetected by either Walsh et al. (2003) using 
SCUBA or at $350 \mu \mathrm{m}$ by van der Tak et al. (2000), as it lies outside both their mapped regions.

G13.19+0.04: panel 73L This field potentially contains three or more sub-mm components; a bright clump to the west $(\mathrm{G} 13.177+0.059 \mathrm{SMM})$, a fainter source to the east (G13.186+0.028SMM) and a bright "ridge" at the northern edge of the SCUBA FOV. This latter emission is not classified as a source in the catalogue due to its truncation by the edge of the SCUBA FOV, which means that the centroid and flux of this source cannot be constrained. Whilst the field contains two compact radio components from the Becker et al. (1994) $5 \mathrm{GHz}$ survey, neither are positionally associated with these sub-mm clumps. Braz \& Epchtein (1983) and Szymczak et al. (2000) report the detection of an $\mathrm{H}_{2} \mathrm{O}$ and $\mathrm{CH}_{3} \mathrm{OH}$ maser respectively toward this region, although the large positional accuracy in their single-dish observations means that the masers cannot be associated with individual sub-mm clumps.

G15.04-0.68: panels $16 \mathrm{~S}$ and $16 \mathrm{~L}$ This region is the well known nebula M17 (also known as the Omega Nebula), an HII region/molecular cloud complex containing a number of early O stars (Nielbock et al. 2001; Hanson et al. 1997). The strongly negative emission features seen in our SCUBA maps (and those of Walsh et al. 2003) imply that the chopping positions were contaminated by sub-mm emission and thus the fluxes measured in this SCUBA field are strictly lower limits to the true flux. The HII region in M17 appears to be viewed as an edge-on blister to the molecular cloud complex, which is in turn comprised of two molecular clouds A \& B (Wilson et al. 2003). The UC HII region identified by WC89a lies in the region M17SW found in cloud $\mathrm{B}$, which is also host to a number of $\mathrm{H}_{2} \mathrm{O}$ masers (Braz \& Epchtein 1983).

G19.61-0.23: panels 21S and 21L This field contains a single centrally peaked sub-mm core, which is known to harbour three embedded compact (3-8" FWHM) ammonia cores and a complex of UC HII regions (Garay et al. 1998).

G20.99+0.09: panels $23 S$ and $23 \mathrm{~L}$ We identify two sub-mm clumps in this field, G20.990+0.089SMM and G20.981+0.094SMM, which comprise a tadpole-like morphology at $850 \mu \mathrm{m}$ with a brighter head (G20.981+0.094SMM) and fainter tail. (G20.990+0.089SMM). The head of the tadpole is unfortunately affected by blanked bolometers and the flux of this component is thus a lower limit to the true flux. This field was a non-detection in the WC89a survey, but Becker et al. (1994) detected three ultracompact radio components at $5 \mathrm{GHz}$ which are found to be mainly associated with the fainter tail of the tadpole. The tail was not detected at $450 \mu \mathrm{m}$.

G23.46-0.20: panels 24S and 24L This field comprises a faint central sub-mm source, G23.454-0.203SMM, and also brighter emission to the SW that is truncated by the edge of the field. G23.454-0.203SMM is positionally coincident with the UC HII region identified by WC89a. The remaining bright source is not included in our catalogue, in view of the impossibility of determining a sensible centroid or flux of this source. Walsh et al. (2003) observed the 450 and $850 \mu \mathrm{m}$ emission from this source (identified in their study as G23.44-0.18), as it is known to contain two $\mathrm{CH}_{3} \mathrm{OH}$ masers (Walsh et al. 1998).
Wider-field ${ }^{13} \mathrm{CO}$ maps of this region reveal the presence of a further molecular clump to the south of G23.44-0.18 (Kim \& Koo 2003).

G23.87-0.12: panels 26S and 26L Four sub-mm clumps are identified in this region, of which only one (G23.861-0.125SMM) is associated with an UC HII region. A further ultracompact $5 \mathrm{GHz}$ radio source was detected in this field by Becker et al. (1994), which lies mid-way between the two sources G23.872-0.122SMM and G23.866-0.114SMM and is positionally associated with neither.

G23.96+0.15: panels $27 \mathrm{~S}$ and 27L We have identified four sub-mm clumps in this field (G23.955+0.148SMM, G23.952+0.152SMM, G23.944+0.159SMM, G23.949 + $0.163 \mathrm{SMM})$. The first two of these clumps form a bright peanutshaped complex in the centre of the image, whilst the latter two are truncated by two blanked bolometers in the final image and may in fact be a single object. This field has also been mapped at $350 \mu \mathrm{m}$ by Hunter et al. (2000), who found a very similar morphology. The two bright clumps in the peanut-shaped complex are designated IRAS 18917-0757 SMM1 and SMM2 by Hunter et al., which corresponds to our SCUBA sources G23.955+0.148SMM and G23.952+0.152SMM respectively. G23.955+0.148SMM is known to be associated with an UC HII region from the WC89a catalogue. Palagi et al. (1993) report the presence of an $\mathrm{H}_{2} \mathrm{O}$ maser associated with this region, but without confirmation at higher angular resolution it is not possible to associate this single-dish maser detection to an individual sub-mm clump.

G25.38-0.18: panel 75L We identify one sub-mm clump in this field, although the two negative "bowls" seen in the image suggest the presence of other nearby clumps in the chopping positions. G25.38-0.18 is also known as the W42 giant HII region and has recently been found to harbour a massive stellar cluster containing a number of YSOs and at least one O5.5 star (Blum et al. 2000). The position of this O5.5 star (W42 1) is close to the peak of the sub-mm clump G25.382-0.184SMM identified in our $850 \mu \mathrm{m}$ image. As the $K$-band spectra of W42 1 (Blum et al. 2000) show that this star has largely cleared away its birth cocoon, this suggests that W42 1 lies in the foreground of G25.382-0.184SMM.

G25.65+1.05: panels $30 \mathrm{~S}$ and 30L This object is also known as RAFGL 7009S and is a well-known embedded massive YSO associated with an UC HII region and displaying strong midinfrared ice absorption features and a powerful molecular outflow (Zavagno et al. 2002; Dartois et al. 2000; Shepherd \& Churchwell 1996; McCutcheon et al. 1995). We identify a single strongly peaked sub-mm clump in the SCUBA 450 and $850 \mu \mathrm{m}$ images, positionally coincident with the UC HII region of KCW94 and with flux levels consistent with those quoted in McCutcheon et al. (1995), allowing for the different filter bandwidths used.

G27.28+0.15: panels $33 S$ and 33L The sub-mm emission from this region takes the form of an elongated filament with 4 associated sub-mm clumps (G27.267+0.146SMM, G27.280+0.144SMM, G27.296+0.151SMM and G27.285+ 0.149 SMM). Only one clump, G27.280+0.144SMM, is 
positionally associated with an UC HII region from the WC89a survey. This clump is also associated with a supernova remnant (SNR 027.3+00.1) that shows $\mathrm{OH}$ maser emission (Green et al. 1997). $\mathrm{CH}_{3} \mathrm{OH}$ maser emission is also detected toward this region by Szycmzak et al. 2000) but with the large positional uncertainties involved in the single-dish detection it is not possible to localise the maser emission within the field. Kim \& Koo $(2001,2003)$ present wider-field maps of this region at 21 $\mathrm{cm}$ and in the ${ }^{13} \mathrm{CO} J=1-0$ line respectively. The $21 \mathrm{~cm}$ radio continuum reveals an extended region of emission elongated to the NW, which in all likelihood represents the SNR. The ${ }^{13} \mathrm{CO}$ map shows an elongated filament of molecular emission extended north-south, consistent with our SCUBA images.

G29.96-0.02: panels 37S and 37L We detected four sub-mm clumps in the SCUBA images of this object, found approximately along a north-south axis. The brightest of these clumps, G29.956-0.017SMM, is associated with the well-known UC HII region and massive star-forming complex G29.96-0.02. This UC HII region has been extensively studied and was the first ultracompact HII region to have its exciting star spectroscopically identified (Watson \& Hanson 1997). The UC HII region has a cometary morphology (WC89a), with a dense, hot molecular core located at the head of the "comet". This hot molecular core has large abundances of $\mathrm{NH}_{3}$ and other saturated molecules (Hatchell et al. 1998; Cesaroni et al. 1994), is positionally associated with $\mathrm{H}_{2} \mathrm{O}$ masers (Hofner \& Churchwell 1996), a compact mid-infrared source (De Buizer et al. 2002) and a possible outflow (Pratap et al. 1999). There is thus strong evidence that this sub-mm clump is an active massive star-forming cluster. There is, so far, no evidence that massive star formation tracers are associated with the other sub-mm clumps in the SCUBA image.

G30.78-0.02: panels 39S and 39L This region is also known as the HII region/molecular cloud complex W43, which has recently been extensively mapped by Motte et al. (2003) at $1.3 \mathrm{~mm}$ and $350 \mu \mathrm{m}$. The two sub-mm sources that we detect towards this region, G30.785-0.022SMM and G30.786-0.028SMM, correspond to the two cores W43 MM6 and W43 MM8 respectively. For a thorough overview of the star formation within W43 the interested reader is referred to Motte et al. (2003).

G33.92+0.11: panels 44S and 44L Although we have identified only a single sub-mm clump in this region (G33.914+0.109SMM), it is clear from the $450 \mu \mathrm{m}$ and $850 \mu \mathrm{m}$ images of this region that the clump has an extension to the west. Higher resolution $\mathrm{C}^{18} \mathrm{O}$ maps of $\mathrm{G} 33.92+0.11$ by Watt \& Mundy (1999) show that G33.914+0.109SMM is indeed comprised of two molecular cores, the fainter of which (Core B) is coincident with the extension seen in the 450 and $850 \mu \mathrm{m}$ images (Fig. 1). As it is not possible to separate the cores in our SCUBA images we include this object as a single sub-mm source in our catalogue.

G34.26+0.15: panels $45 \mathrm{~S}$ and $45 \mathrm{~L}$ This region is arguably one of the most well-studied massive star-forming regions in the Galaxy. The G34.26+0.15 region is a molecular cloud/HII region complex comprising a large blister HII region to the SE of a kidney-shaped molecular clump approximately $3.5 \times 4 \mathrm{pc}$ in size (Carral \& Welch 1992; Heaton et al. 1985). Embedded within this molecular clump are a hot molecular core that exhibits strong $\mathrm{H}_{2} \mathrm{O}$ maser emission (Hofner \& Churchwell 1996) and large abundances of saturated molecules (Macdonald et al. 1996), two unresolved UC HII regions and a spectacular cometary HII region with a clearly defined tail stretching for at least $20^{\prime \prime}$ outwards from the centre of the molecular clump to the west (Gaume et al. 1994). Surprisingly the sub-mm morphology follows the appeareance of the cometary HII region, although at a much larger scale. The sub-mm emission has a broad cometary morphology with little emission to the west of the main core, suggesting that the cometary HII region may be expanding into this low-density region. We identify three sub$\mathrm{mm}$ sources in the SCUBA images; the central clump detailed above (G34.257+0.152SMM), an elongated clump to the northwest, G34.257+0.164SMM, that is positionally coincident with an $\mathrm{H}_{2} \mathrm{O}$ maser (Fey et al. 1994) and a further clump to the southeast, G34.244+0.133SMM, previously discovered at $350 \mu \mathrm{m}$ by Hunter et al. (1998) and identified as a candidate embedded proto-B star.

G43.80-0.13: panels $50 \mathrm{~S}$ and $50 \mathrm{~L}$ This object is a wellknown $\mathrm{OH}$ maser source known as $\mathrm{OH} 43.8-0.1$ (Braz \& Epchtein 1983), which also harbours variable $\mathrm{H}_{2} \mathrm{O}$ maser spots (Lehkt 2000). The SCUBA images reveal that the sub-mm clump is elongated roughly north-south, which is not consistent with the recent CS $J=5-4$ map of Shirley et al. (2003), in which the CS clump has a moderate east-west extension.

G45.07+0.13/G45.12+0.13: panels 51S and 51L and 52S and $52 \mathrm{~L}$ The molecular environment of both these UC HII regions was the focus of a thorough study by Hunter et al. (1997), who found that both of these UC HII regions are embedded in molecular clumps massing several thousand $M_{\odot}$ and are associated with bipolar outflows. Our sub-mm images agree closely with those obtained by Hunter et al. (1997), including the east-west extension seen in the $450 \mu \mathrm{m}$ image of G45.07+0.13.

G45.47+0.05: panel 87L This field contains two UC HII regions, G45.47+0.05 and G45.45+0.06, which are both associated with compact $850 \mu \mathrm{m}$ emission (Fig. 2). This latter UC HII region is also associated with a cluster of massive YSOs (Feldt et al. 1998).

G69.54-0.98: panels 53S and 53L This region is also known as ON 1 (Winnberg 1970) or OH 69.5-1.0 and is associated with a well-known $\mathrm{OH}$ maser (Braz \& Epchtein 1983) and $\mathrm{H}_{2} \mathrm{O}$ maser emission (Palagi et al. 1993).

G70.29+1.60: panels 54S and 54L This region is also known as $\mathrm{K} 3-50 \mathrm{~A}$ and is a well-known UC HII region residing within the star-forming complex W58. The ionised gas comprising $\mathrm{K} 3-50 \mathrm{~A}$ displays a strong velocity gradient that is suggestive of a bipolar ionised flow (de Pree et al. 1994). This ionised flow is surrounded by a massive molecular torus $\sim 0.5 \mathrm{pc}$ in radius, in turn embedded within a larger lower-density molecular clump some $1.1 \mathrm{pc}$ in radius (Howard et al. 1997). High resolution mid-infrared spectroscopic imaging has recently revealed that the UC HII region is in fact ionised by multiple O stars (Okamoto et al. 2003). 
G76.38-0.62: panels 56S and 56L This object is the wellknown bipolar HII region S 106 (Sharpless 1959, an hourglass shaped HII region oriented north-south with a dark dust lane running from east to west across the centre of the HII region (e.g. Smith et al. 2001). The HII region is extended over roughly $3 \times 2^{\prime}$ and it is likely that the UC HII region identified toward this source by KCW94 represents stellar wind emission from the central ionising star. The SCUBA maps of G76.38-0.62 in Fig. 1 clearly show the presence of the dark dust lane across the centre of S 106 and closely resemble those of Richer et al. (1993), to which the reader is referred for a fuller discussion of the nature of S 106.

G77.97-0.01: panels 57S and 57L The sub-mm emission in this field takes the form of two diffuse clumps, of which the clump at the field centre (G77.962-0.008SMM) is associated with the KCW94 UC HII region G77.965-0.006 and an infrared stellar cluster (Bica et al. 2003). The compact VLA configuration observations of Kurtz et al. (1999) reveal that there is an extended diffuse component of 3.6 and $20 \mathrm{~cm}$ emission, peaking on both sub-mm clumps and with an extended more diffuse tail stretching to the east.

G106.80+5.31: panels $62 \mathrm{~S}$ and $62 \mathrm{~L}$ This region is the wellknown S 140 HII region/molecular cloud complex which is known to harbour at least three embedded infrared protostars, multiple outflows and a variable $\mathrm{H}_{2} \mathrm{O}$ maser (e.g. Bally et al. 2002; Minchin et al. 1995; Brand et al. 2003). G106.80+5.31 was mapped in the sub-mm by Minchin et al. (1995) and our SCUBA images agree closely with these maps, although we could not deblend the two sources S 140 SMM1 and SMM3. These sources are represented by our single sub$\mathrm{mm}$ clump G106.794+5.313SMM. The remaining sub-mm clump in the field, G106.800+5.314SMM corresponds to the source S 140 SMM2 from Minchin et al. (1995).

G109.87+2.11: panels 63S and 63L This region is also known as Cep A and is an extremely well-studied molecular cloud (Sargent 1977, 1979) exhibiting vigorous signs of star formation including embedded UC HII regions, YSOs, protostars, outflows and $\mathrm{H}_{2} \mathrm{O}$ masers (e.g. Richardson et al. 1987; Moriarty-Schieven et al. 1991; Torrelles et al. 1993; Rodriguez et al. 1994; Gómez et al. 1999; Hughes 2001). We detect a single sub-mm clump in this field with a bright central core associated with lower brightness extended emission along a NE-SW axis. The extended sub$\mathrm{mm}$ emission is elongated along almost the same axis as the string of UC HII regions observed toward Cep A (Hughes \& Wouterloot 1984). The fluxes measured from our SCUBA images are consistent with those measured by Moriarty-Schieven et al. (1991).

G110.21+2.63: panel 88L A single diffuse clump of sub-mm emission is detected in this field (G110.211+2.609SMM) at $850 \mu \mathrm{m}$ only. This region is also known as the Cep B molecular cloud, which is found along with Cep A in the Cep OB3 molecular cloud complex (Sargent 1977, 1979). Cep B is found to be associated with a cluster of $\mathrm{T}$ Tauri candidates (Moreno-Corral et al. 1993). Testi et al. (1995) identify three HII regions toward Cep B, a blister HII region some $45 \times 25^{\prime \prime}$ in extent ([TOF95] A) and two ultracompact HII regions ([TOF95] B and $\mathrm{C})$. Component $\mathrm{B}$ is coincident with the UC HII region
G110.209+2.630 identified by KCW94. All of these HII regions are displaced from the sub-mm peak of G110.211+2.609SMM.

G111.28-0.66: panels 64S and 64L This region is also known as the S 157 HII region (Sharpless 1959). The UC HII region identified by KCW 94 (G111.282-0.663) is associated with an extended HII region to the NE $\sim 1^{\prime}$ in radius and a further compact or ultracompact region to the SW (Kurtz et al. 1999). We identify two sub-mm clumps in our SCUBA image (G111.282-0.665SMM and G111.281-0.670SMM), of which the first is positionally associated with the UC HII region identified by KCW94 and the second is associated with the compact HII region identified by Kurtz et al. (1999).

G111.61+0.37: panels 65S and 65L This region is also known as the HII region/molecular cloud complex S 159. KCW94 identified a single UC HII region in this field, positionally coincident with the bright sub-mm peak seen in our SCUBA images. Kurtz et al. (1999) report the detection of extended $21 \mathrm{~cm}$ emission to the east of the UC HII region and a further compact component is reported to the west of the UC HII region by Lebrón et al. (2001). This latter component is positionally coincident with the NW spur of emission seen in the SCUBA $850 \mu$ m image.

G133.95+1.06: panels 66S and 66L This region is the wellknown star-forming region $\mathrm{W} 3(\mathrm{OH})$ found in the $\mathrm{W} 3$ starforming complex (Wynn-Williams et al. 1974). The UC HII region observed by KCW94 is found to split up into multiple sources at higher resolution (Baudry et al. 1988) and W3 (OH) is also known to harbour a compact cluster of infrared sources (Bica et al. 2003) and three compact cores, collectively known as $\mathrm{W} 3\left(\mathrm{H}_{2} \mathrm{O}\right)$, detected by their millimetre-wave continuum and $\mathrm{H}_{2} \mathrm{O}$ maser emission (Wyrowski et al. 1999, 1997). The sub-mm peak detected in our SCUBA map (G133.949+1.064SMM) is coincident with the position of $\mathrm{W} 3\left(\mathrm{H}_{2} \mathrm{O}\right)$. For a recent overview of star formation in the $\mathrm{W} 3$ region the interested reader is referred to Tieftrunk et al. (1998).

G188.79+1.03/G188.77+0.92: panel 89L Both of these UC HII regions lie within the S 257 HII region complex, which was recently imaged in the far-infrared by Ghosh et al. (2000). The far-infrared maps suggest that G188.79+1.03 may be extended in an east-west direction, which is consistent with the low-level $(3 \sigma)$ emission seen to the west of the main clump in our SCUBA $850 \mu \mathrm{m}$ image (see Fig. 2). G188.77+0.92 was not detected in the sub-mm during this survey and is not shown in Figs. 1 or 2. Both G188.79+1.03 and G188.77+0.92 are known to be associated with molecular outflows (Shepherd \& Churchwell 1996).

G213.88-11.84: panel 91L This region is also known as GGD 14 (Gómez et al. 2000) and, in addition to the cometary UC HII region detected by KCW94, hosts a cluster of ultracompact radio sources that are potentially low-mass pre-mainsequence stars (Gómez et al. 2002). G213.88-11.84 is associated with a molecular outflow (Little et al. 1990). We identify a single sub-mm clump in our SCUBA $850 \mu \mathrm{m}$ image, although the clump is extended toward the NE (in the direction of the radio source [GRG2002] VLA 7) which may indicate the presence of unresolved substructure in our image. 


\section{Analysis and discussion}

During the survey we identified three distinct types of object: ultracompact $\mathrm{cm}$-wave sources that are not associated with any sub-mm emission ("sub-mm quiet objects"), sub-mm clumps that are associated with ultracompact $\mathrm{cm}$-wave sources ("radioloud clumps"); and sub-mm clumps that are not associated with any known ultracompact cm-wave sources ("radio-quiet clumps"). In this section we investigate the likely nature of each kind of object. We also examine the broad properties of the sample as a whole, focusing upon their morphology and clustering properties. We postpone a detailed analysis of the physical properties of each sub-mm clump to a later paper in the series (Thompson et al. 2006), where we present an in-depth study of the temperature, mass and density of each sub-mm clump.

\section{1. "Sub-mm quiet" ultracompact radio sources: are they UC HII regions?}

From an inspection of Figs. 1 and 2, it is clear that the vast majority of ultracompact radio sources investigated in this study are positionally associated with bright sub-mm emission and are thus likely to be young UC HII regions embedded within molecular cloud clumps. Nevertheless we have identified a substantial population of ultracompact radio sources in the fields surveyed that are not associated with any detected sub-mm emission. For brevity we hence refer to these objects as "sub-mm quiet objects". What is the likely nature of these sub-mm quiet objects? The standard model of an UC HII region is a massive YSO embedded within a dusty molecular cocoon that is optically thick to the visible-UV radiation of the YSO. The compact cm-wave radiation results from free-free emission from the ionised gas of the UC HII region but the bulk of the radiation is emitted in the sub-mm, far- and mid-infrared regions through dust reprocessing of the shorter-wavelength radiation by the optically thick cocoon (e.g. Crowther \& Conti 2003; Churchwell 2002). Following this picture sub-mm quiet objects are unlikely to be associated with massive dust cocoons and thus may not be true UC HII regions.

We investigate this possibility by constraining the upper limit to the mass of any molecular clump associated with the sub-mm quiet objects. The VLA surveys possess sufficient sensitivity to detect UC HII regions excited by B0 stars out to a distance of $20 \mathrm{kpc}$ (Giveon et al. 2005) but the sensitivity of our SCUBA survey is such that we would not detect clumps of less than a few hundred solar masses at this distance. There is thus the potential that the sub-mm quiet objects may represent a population of distant UC HII regions embedded in relatively low mass clumps.

In the following discussion we refer only to the $850 \mu \mathrm{m}$ data, as the signal-to-noise ratio of these images is typically a factor or 10 higher that that at $450 \mu \mathrm{m}$. A total of 80 radio sources identified from the literature search described in Sect. 2.1 were found to be unassociated with $850 \mu \mathrm{m}$ emission to a level of $3 \sigma$, or a typical flux limit of $0.4-0.6 \mathrm{Jy} / \mathrm{beam}$. These sub-mm quiet objects are listed in Table 4 along with their corresponding SCUBA field, the $850 \mu \mathrm{m}$ flux upper limit, the distance to the radio component (if known) and an upper limit to the mass of any molecular clump that could be associated with the sub-mm quiet objects. Masses were calculated using the method outlined in Hildebrand (1983) assuming the upper flux limits and distances given in Table 4, a dust temperature of $30 \mathrm{~K}$, dust emissivity $\beta=2$ and a mass coefficient of $C_{850 \mu \mathrm{m}}=50 \mathrm{~g} \mathrm{~cm}^{-2}$ (from Kerton et al. 2001). For cases where a kinematic distance ambiguity exists we have calculated the mass upper limits at both the near and far distances. We have also assumed that each sub-mm quiet object in a single SCUBA field lies at the same distance.

It can be seen from Table 4 that the $850 \mu \mathrm{m}$ flux upper limits preclude high mass clumps toward all but the furthest sub-mm quiet objects. Objects closer than a distance of $10 \mathrm{kpc}$ cannot be embedded in clumps with masses in excess of a few tens of solar masses. The most extreme source in Table 4 is [KCW94] $110.209+2.630$ which at an assumed distance of $0.7 \mathrm{kpc}$ has an upper limit to the mass of an associated molecular clump of only $0.3 M_{\odot}$. Even at distances greater than $10 \mathrm{kpc}$ the masses of any associated molecular clumps are only a few hundred solar masses $\left(472 M_{\odot}\right.$ in the furthest object in the sample; [GBH05] 6.55647-0.09042). This mass limit is close to the corresponding median mass of the detected sub-mm clumps in the sample; the median flux and distance in our sample of detected sub-mm clumps is $7 \mathrm{Jy}$ and $5.2 \mathrm{kpc}$, corresponding to a median mass of $530 M_{\odot}$. It is possible that those sub-mm quiet objects with distances in excess of $10 \mathrm{kpc}$ may be part of a relatively low clump-mass population of embedded UC HII regions.

If the nearby sub-mm quiet objects with distances less than $10 \mathrm{kpc}$ are embedded within molecular cloud clumps then these clumps must possess masses of less than a few tens of solar masses. There is considerable uncertainty regarding the minimum mass cloud clump required to form a high mass star, some studies suggest star formation efficiencies of 5-10\% (Clark et al. 2005) or 10-30\% (Lada \& Lada 2003, and references therein). One thus might naively expect that a $10 M_{\odot}$ star should only form in a clump of at least 30-200 $M_{\odot}$. Observational values for the mass of high-mass star-forming clumps are at the higher end of the scale, ranging from $720 M_{\odot}$ (Mueller et al. 2002) to $10^{4} M_{\odot}$ (Hatchell et al. 2000). The masses of the sub-mm quiet objects are thus below the lower mass end of the observational scale and close to the minimum clump mass suggested by a simple consideration of star formation efficiency. We thus conclude that it is unlikely that sub-mm quiet objects nearer than $10 \mathrm{kpc}$ are true UC HII regions, unless they are embedded within extremely low-mass clumps.

It is possible that the sub-mm quiet objects represent planetary nebulae, which are known to emit $\mathrm{cm}$-wave radiation at the mJy level and possess relatively low-mass dust envelopes that could fall below our SCUBA detection limits. An alternate explanation is suggested by the fact that in a significant number of our SCUBA survey fields (10/32 fields) the sub$\mathrm{mm}$ quiet sources are strongly clustered, particularly objects from the Giveon et al. (2005) survey. Moreover, the majority of these "clusters" are often found at the peripheries of radioloud or radio-quiet sub-mm clumps, for example G19.07-0.27 in panel 19L, G27.49+0.19 in panel 34L and G28.60-0.36 in panel 76L of Fig. 1. The Giveon et al. survey, like all of the VLA surveys described in this paper, is a snapshot interferometer survey with limited $u v$ coverage that significantly limits the largest angular scale visible in the survey data. The bright components of "resolved-out" large angular scale structures often appear as clusters of apparent point sources and it is possible that the clustered Giveon et al. (2005) objects trace bright knots within extended structures rather than clusters of UC HII regions.

This hypothesis is bolstered by the fact that a number of the clustered Giveon et al. objects are correlated with extended MSX $8 \mu \mathrm{m}$ emission signifying PAH emission from the fringes of an extended HII region. As an example we present MSX $8 \mu \mathrm{m}$ and NRAO VLA Sky Survey (NVSS) $20 \mathrm{~cm}$ images of the SCUBA field G19.07-0.27 in Fig. 3. NVSS data were used by Kurtz et al. (1999) to identify extended continuum emission toward a small sample of UC HII regions, as the longer 
Table 4. Ultracompact radio components in the survey fields with undetected sub-mm emission to a level of $3 \sigma$. The radio sources are indicated by their SIMBAD identifier; [WC89] for the WC89a survey; [KCW94] for the KCW94 survey, [GPSR5] for the Becker et al. (1994) 5GHz Galactic Plane survey; [GPSR] for the Becker et al. (1990) and Zoonematkermani et al. (1990) $1.4 \mathrm{GHz}$ Galactic Plane survey and [GBH05] for the work of Giveon et al. (2005). Upper limits to the clump mass have been calculated from the $850 \mu \mathrm{m}$ flux upper limit using the assumptions outlined in Sect. 4.1. An ellipsis is given for those radio components of unknown distance while for objects with distance ambiguities both near and far distances and clump masses are given.

\begin{tabular}{|c|c|c|c|c|c|}
\hline UC HII field & \multicolumn{2}{|c|}{ Known ultracompact radio components } & $850 \mu \mathrm{m}$ flux limit & Distance & Clump mass limit \\
\hline G5.48-0.24 & [GBH05] 5.48464-0.23059 & & 0.4 & 14.3 & 219 \\
\hline G6.55-0.10 & [GBH05] 6.55647-0.09042 & & 0.6 & 16.7 & 472 \\
\hline G8.67-0.36 & $\begin{array}{l}\text { [GBH05] 8.66530-0.34198 } \\
\text { [GBH05] 8.66202-0.34038 }\end{array}$ & $\begin{array}{l}\text { [GBH05] 8.66324-0.34337 } \\
\text { [GBH05] 8.66730-0.34437 }\end{array}$ & 0.9 & 4.6 & 53 \\
\hline G10.30-0.15 & $\begin{array}{l}\text { [WC89] 010.30-0.15A } \\
\text { [GBH05] } 10.30485-0.14319 \\
\text { [GBH05] } 10.30605-0.14551\end{array}$ & $\begin{array}{l}\text { [GBH05] 10.30726-0.14585 } \\
\text { [GBH05] 10.32159-0.15542 } \\
\text { [GBH05] } 10.30485-0.14319\end{array}$ & 0.6 & 6.0 & 65 \\
\hline $\begin{array}{l}\text { G10.62-0.38 } \\
\text { G110.21+2.63 } \\
\text { G13.19+0.04 }\end{array}$ & $\begin{array}{l}{[\mathrm{GBH} 05] 10.59905-0.38336} \\
{[\mathrm{KCW} 94] 110.209+2.630} \\
{[\mathrm{GBH} 05] 13.19014+0.04077} \\
{[\mathrm{GBH} 05] 13.19014+0.04077}\end{array}$ & $\begin{array}{l}\text { [GBH05] 10.59905-0.38336 } \\
{[\mathrm{GBH} 05] 13.18643+0.04999}\end{array}$ & $\begin{array}{l}0.9 \\
0.2 \\
0.4\end{array}$ & $\begin{array}{c}4.8 \\
0.7 \\
4.4 / 12.1\end{array}$ & $\begin{array}{c}57 \\
0.3 \\
21 / 161\end{array}$ \\
\hline $\begin{array}{l}\text { G13.87+0.28 } \\
\text { G18.15-0.28 } \\
\text { G19.07-0.27 }\end{array}$ & $\begin{array}{l}\text { [GBH05] 13.87696+0.28238 } \\
\text { [GBH05] 18.15158-0.28016 } \\
\text { [WC89] 019.07-0.27 } \\
\text { [GBH05] 19.06256-0.27062 } \\
\text { [GBH05] 19.07084-0.27694 }\end{array}$ & $\begin{array}{l}{[\mathrm{GBH} 05] 13.87595+0.28479} \\
{[\mathrm{GBH} 05] 18.14744-0.29764} \\
{[\mathrm{GBH} 05] 19.06392-0.27384} \\
{[\mathrm{GBH} 05] 19.06732-0.28637}\end{array}$ & $\begin{array}{l}0.6 \\
0.7 \\
0.5\end{array}$ & $\begin{array}{l}4.5 \\
4.2 \\
5.4\end{array}$ & $\begin{array}{l}35 \\
34 \\
40\end{array}$ \\
\hline G19.49+0.14 & $\begin{array}{l}\text { [GBH05] } 19.48265+0.16098 \\
{[\mathrm{GBH} 05] 19.48283+0.15668} \\
{[\mathrm{GBH} 05] 19.48468+0.15613}\end{array}$ & $\begin{array}{l}\text { [GBH05] 19.48660+0.15574 } \\
\text { [GBH05] } 19.49724+0.13390\end{array}$ & 0.3 & $2.0 / 14.0$ & $3 / 145$ \\
\hline $\begin{array}{l}\text { G20.08-0.14 } \\
\text { G23.46-0.20 }\end{array}$ & $\begin{array}{l}\text { [GBH05] 20.07849-0.14609 } \\
{[\text { WC89] 023.46-0.20A }} \\
{[\text { GBH05] 23.43821-0.20869 }} \\
{[\text { GBH05] 23.43876-0.21157 }} \\
{[\text { GBH05] 23.44160-0.21151 }}\end{array}$ & $\begin{array}{l}\text { [GBH05] 23.43595-0.20366 } \\
{[\mathrm{GBH} 05] 23.43876-0.21157} \\
{[\mathrm{GBH} 05] 23.44160-0.21151}\end{array}$ & $\begin{array}{l}0.2 \\
0.4\end{array}$ & $\begin{array}{c}12.3 \\
9.0\end{array}$ & $\begin{array}{l}71 \\
82\end{array}$ \\
\hline $\begin{array}{l}\text { G24.68-0.16 } \\
\text { G26.10-0.07 } \\
\text { G25.72+0.05 } \\
\text { G27.28+0.15 }\end{array}$ & $\begin{array}{l}{[\mathrm{GBH} 05] 24.67878-0.15397} \\
{[\mathrm{GBH} 05] 26.09201-0.05675} \\
{[\mathrm{GBH} 05] 25.69526+0.03343} \\
{[\mathrm{GBH} 05] 27.28030+0.16537} \\
{[\mathrm{GBH} 05] 27.27070+0.14850} \\
{[\mathrm{GBH} 05] 27.28271+0.16406}\end{array}$ & $\begin{array}{l}\text { [GBH05] } 25.69562+0.03499 \\
{[\mathrm{GBH} 05] 27.27497+0.15800} \\
{[\mathrm{GBH} 05] 27.27809+0.14873} \\
{[\mathrm{GBH} 05] 27.27569+0.14696}\end{array}$ & $\begin{array}{l}0.5 \\
0.4 \\
0.2 \\
0.5\end{array}$ & $\begin{array}{l}6.3 / 9.1 \\
\ldots \\
14.0 \\
15.2\end{array}$ & $\begin{array}{c}56 / 117 \\
\ldots \\
120 \\
338\end{array}$ \\
\hline $\mathrm{G} 27.49+0.19$ & $\begin{array}{l}\text { [GBH05] 27.49356+0.19212 } \\
{[\text { GBH05] 27.49556+0.19105 }}\end{array}$ & [GBH05] 27.49617+0.19389 & 0.4 & $2.5 / 12.6$ & $7 / 168$ \\
\hline G28.60-0.36 & $\begin{array}{l}\text { [GBH05] 28.59890-0.36738 } \\
{[\mathrm{GBH} 05] 28.59173-0.36057} \\
{[\mathrm{GBH} 05] 28.59560-0.36862}\end{array}$ & $\begin{array}{l}\text { [GBH05] 28.60723-0.36382 } \\
\text { [GBH05] 28.60375-0.36693 }\end{array}$ & 0.3 & $5.2 / 9.7$ & $22 / 75$ \\
\hline $\begin{array}{l}\text { G28.80+0.17 } \\
\text { G28.83-0.23 }\end{array}$ & $\begin{array}{l}\text { [GBH05] 28.79740+0.17314 } \\
\text { [GBH05] 28.82159-0.22657 } \\
\text { [GBH05] 28.81948-0.22485 }\end{array}$ & $\begin{array}{l}\text { [GBH05] 28.80450+0.16777 } \\
\text { [GBH05] 28.81780-0.22737 }\end{array}$ & $\begin{array}{l}0.5 \\
0.4\end{array}$ & $\begin{array}{l}6.4 / 8.5 \\
5.1 / 9.8\end{array}$ & $\begin{array}{l}62 / 109 \\
30 / 111\end{array}$ \\
\hline $\begin{array}{l}\text { G330.78-0.02 } \\
\text { G33.13-0.09 }\end{array}$ & $\begin{array}{l}\text { [GBH05] 30.78458-0.01577 } \\
\text { [GBH05] 33.14311-0.08555 }\end{array}$ & & $\begin{array}{l}0.8 \\
0.2\end{array}$ & $\begin{array}{l}5.5 \\
7.1\end{array}$ & $\begin{array}{l}70 \\
34\end{array}$ \\
\hline G37.36-0.23 & [GPSR5] 37.356-0.232 & [GBH05]37.35747-0.23191 & 0.4 & & $\ldots$ \\
\hline G37.77-0.20 & [GBH05] 37.76595-0.19983 & [GBH05] 37.76913-0.20613 & 0.4 & 8.8 & 83 \\
\hline G39.25-0.06 & $\begin{array}{l}\text { [GBH05] 39.25972-0.05001 } \\
\text { [GBH05] 39.25972-0.05001 }\end{array}$ & [GBH05] 39.24685-0.06521 & 0.4 & 11.4 & 160 \\
\hline $\begin{array}{l}\text { G41.52-0.04 } \\
\text { G41171+0.11 }\end{array}$ & $\begin{array}{l}\text { [GPSR5] 41.525+0.039 } \\
\text { [WC89] } 04171+0.11\end{array}$ & GPSR5 41.507+0.030 & $\begin{array}{l}0.2 \\
0.2\end{array}$ & $\cdots$ & $\cdots$ \\
\hline $\begin{array}{l}\text { G41.71+0.11 } \\
\text { G42.42-0.27 }\end{array}$ & [WC89] 042.42-0.27B & & $\begin{array}{l}0.2 \\
0.3\end{array}$ & $\dddot{5.2}$ & $\dddot{21}$ \\
\hline G42.90+0.57 & [WC89] 042.90+0.57A & [WC89] 042.90+0.57B & 0.3 & 5.2 & 21 \\
\hline $\mathrm{G} 44.26+0.10$ & [GPSR] 44.261+0.104 & & $>0.1$ & $\ldots$ & $\ldots$ \\
\hline $\mathrm{G} 188.77+1.07$ & [KCW94] 188.770+01.074 & & 0.2 & $\ldots$ & $\ldots$ \\
\hline
\end{tabular}

wavelength and more compact VLA configuration of NVSS considerably improves its sensitivity to large-scale structure. Figure 3 reveals that the sub-mm clump G19.077-0.289SMM lies at the eastern edge of an extended region of $8 \mu \mathrm{m}$ and $20 \mathrm{~cm}$ emission, which probably marks the position of an extended HII region. The cluster of Giveon et al. (2005) objects lies within 

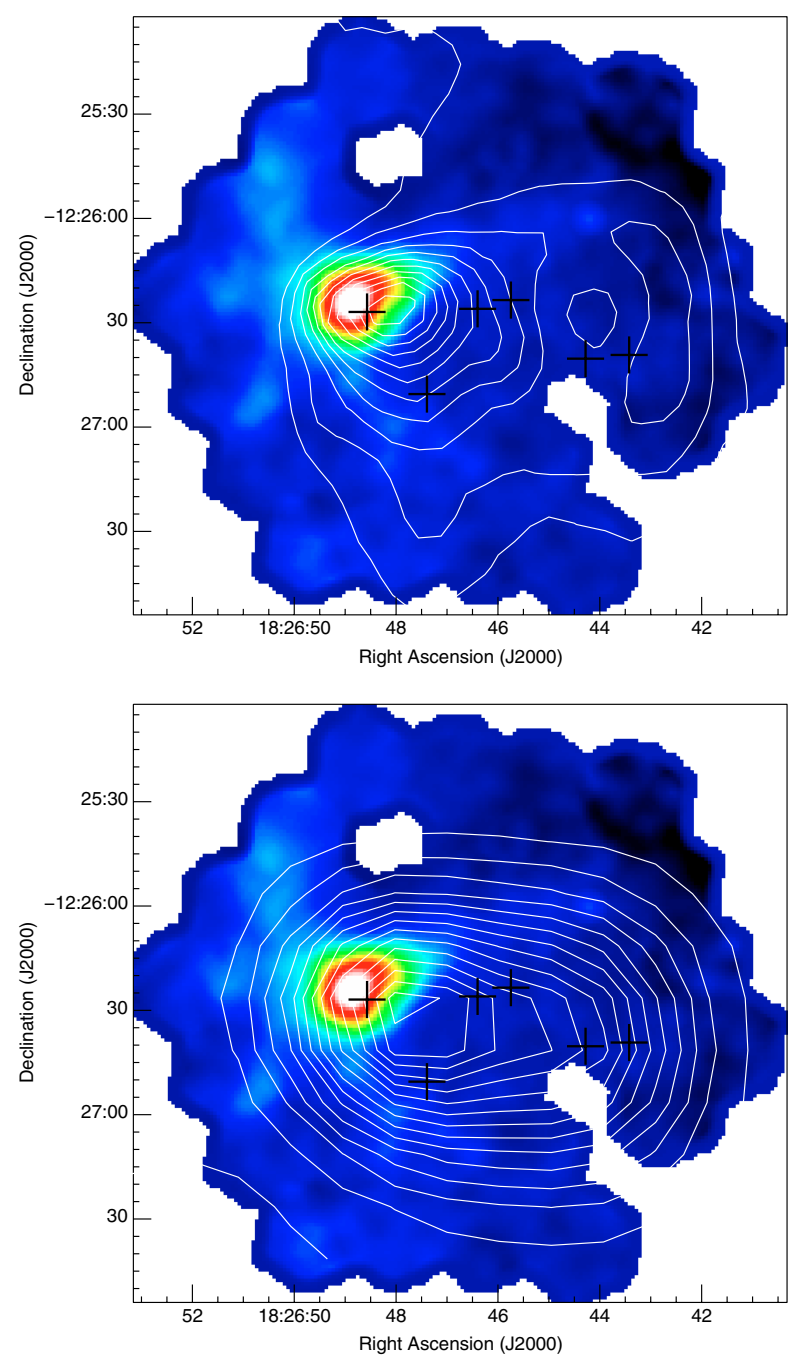

Fig. 3. $850 \mu \mathrm{m}$ SCUBA images of the UC HII field G19.07-0.27 overlaid with countours of MSX $8 \mu \mathrm{m}$ emission (top) and NVSS $20 \mathrm{~cm}$ emission (bottom). Crosses indicate the position of ultracompact radio components identified from the WC89a, Becker et al. (1994) and Giveon et al. (2005) surveys.

this extended HII region and are more than likely bright components of the extended free-free emission of the HII region rather than individual UC HII regions.

The overall appearance of the G19.07-0.27 region in the sub-mm, mid-IR and radio is consistent with the hierarchical structure hypothesis of Kim \& Koo $(2001,2003)$ for HII regions with both ultracompact and extended components. As seen in Fig. 3 the "sub-mm loud" ultracompact radio source associated with G19.077-0.289SMM lies to the south-west of the sub-mm peak, on the same face as the extended mid-IR and radio emission traced by MSX and NVSS. It is thus possible that the sub$\mathrm{mm}$ loud ultracompact component corresponds to the position of an embedded O-star(s) whose HII region is generally constrained by the high ambient clump density within the clump (and hence has an ultracompact component) but has broken free from the clump into the lower density region to the west to form a champagne flow HII region. It is unclear what fraction of the ultracompact cm-wave sources contained in the WC89a, KCW94 or Giveon et al. (2005) catalogues are extended objects similar to G19.07-0.27. Nevertheless the Kim \& Koo (2001) hierarchical structure hypothesis offers a plausible explanation for these clustered sub-mm quiet objects. Further investigation of the UC HII region catalogues is necessary to determine the fraction of extended interlopers in the present UC HII region catalogues and whether the extended HII regions are consistent with the Kim \& Koo model.

\subsection{Radio-loud and radio-quiet sub- $m m$ clumps}

The sub-mm clumps that were detected in the survey split into two types; those that are positionally associated with ultracompact radio sources and those that are not associated with any detected ultracompact radio sources. The former are referred to as "radio-loud" clumps and the latter as "radio-quiet" clumps. We define positionally associated as the position of the radio source lying within the lowest $850 \mu \mathrm{m}$ contour bounding the clump (the lowest contour corresponds to the $3 \sigma$ level in each image, see Sect. 3.1). It is important to note that all of our target fields have been observed with the VLA as part of the WC89a, KCW94, Becker et al. (1994) or Giveon et al. (2005) surveys and so the radio-quiet clumps are truly radio-quiet (to within the flux limits set by the VLA surveys) rather than simply radio-unobserved. We discuss the likely individual nature of the radio-loud and radio-quiet clumps in more detail in Sects. 4.3 and 4.5 but in this section we will briefly dwell upon the properties of the population of sub-mm clumps as a whole.

\subsubsection{Sub-mm colours and "luminosities"}

The sub-millimetre wavelength radiation detected from the clumps originates from thermal emission from dust grains contained within the molecular gas of the clumps and is an excellent optically thin tracer of the column density within the clumps (e.g. Hildebrand 1983). The wavelength dependence of the submm emission depends upon both the temperature of the dust and its emissivity coefficient $\beta$. The standard procedure in the determination of the column density (or mass) of a sub-mm clump, its temperature and grain emissivity is to fit a greybody model to the broad spectrum SED of the clump (e.g. Dent et al. 1998). Usually a combination of IRAS far-infrared and millimetre/submillimetre measurements provide the SED, but for many of the clumps detected in our survey (particularly those SCUBA fields with multiple sub-mm components) the IRAS data is hopelessly confused due to the large beam at $100 \mu \mathrm{m}\left(\sim 2^{\prime}\right.$ FWHM).

As part of Paper II we present greybody and radiative transfer analyses for those clumps that are not confused over the large scale of the IRAS beam, but here we present an investigation of the sub-mm properties of the wider sample as a whole. One of the standard techniques used to investigate the properties of large stellar samples is the colour-magnitude diagram. This approach is not a standard tool in the study of star-forming cores, but was recently adapted by Crowther \& Conti (2003) to investigate the mid-infrared properties of UC HII regions. We adapt their technique for the sub-millimetre by using the distance-scaled $850 \mu \mathrm{m}$ flux of the clumps as a surrogate for the clump luminosity and the $850 \mu \mathrm{m} / 450 \mu \mathrm{m}$ flux ratio for the sub-mm colour, renaming the approach as a colour-luminosity diagram to avoid confusion.

Assuming that clumps are optically thin at both 450 and $850 \mu \mathrm{m}$, their sub-mm colour should depend solely upon the source-averaged dust temperature and grain emissivity. Thus by plotting a sub-mm colour-luminosity diagram we are able to investigate different regions in the dust temperature/emissivity parameter space as a function of clump luminosity. The sub-mm colour-luminosity diagram of the sub-mm clumps detected in 


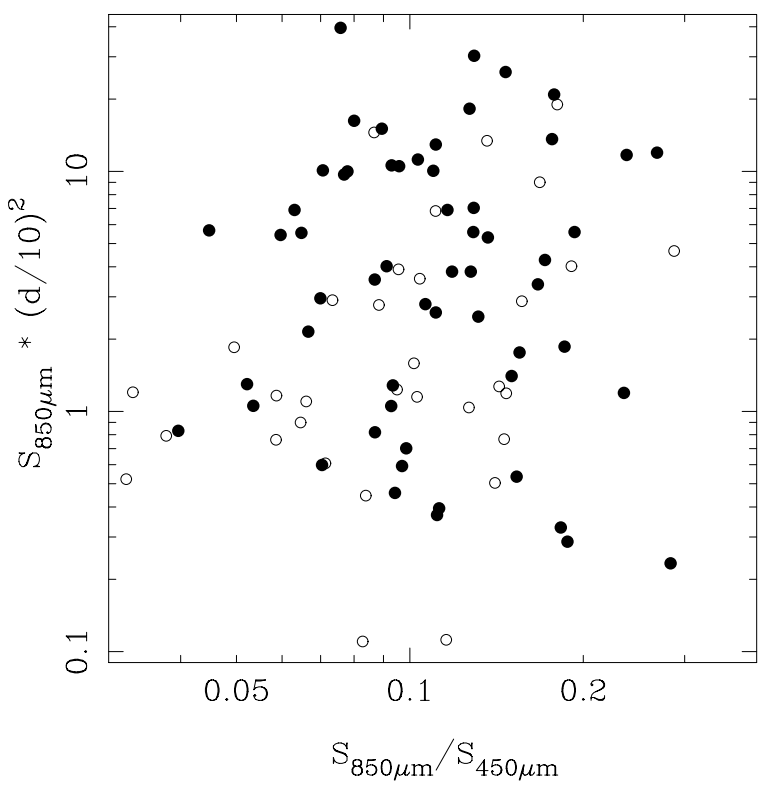

Fig. 4. Sub-mm "colour-luminosity" diagram. Radio-loud clumps are indicated by filled circles and radio-quiet clumps are shown by open circles.

the survey is shown in Fig. 4. Only those clumps with unambiguous distances are included in Fig. 4. As many of the radioquiet clumps lack known kinematic distances we assume that radio-quiet clumps located within the same SCUBA field as a radio-loud clump with a known distance lie at that known distance (i.e. the radio-loud and radio-quiet clumps are part of the same complex).

Two insights are immediately apparent from the colourluminosity diagram shown in Fig. 4. The first is that, in general, the radio-loud clumps tend to have a higher distance-scaled $850 \mu \mathrm{m}$ flux than the radio-quiet clumps. The median distancescaled $850 \mu \mathrm{m}$ flux for radio-loud clumps is $3.8 \mathrm{Jy}$, compared to $1.2 \mathrm{Jy}$ for the radio-quiet clumps. A Kolmogorov-Smirnov test also indicates that the two distance-scaled flux distributions are unlikely to be drawn from the same population, with a probability of only $2.5 \%$ for a single flux distribution hypothesis. This may imply that the radio-quiet clumps form a separate lower-luminosity population to the radio-loud UC HII regioncontaining clumps (note that the typical UC HII detection limit from WC89a is of a B0.5 star at $5 \mathrm{kpc}$ ). The radio-quietness of these clumps may perhaps thus be due to the fact that the clumps are forming lower-mass stars rather than high-mass protostars in a pre-UC HII region phase. It must however be cautioned that, as the radio-quiet sample is almost certainly comprised of a mixed population of massive star-forming clusters in a pre-UC HII phase and low- to intermediate-mass star-forming clusters, it is impossible to make conclusions about the nature of the entire sample of radio-quiet clumps from this data.

The second insight from the sub-mm colour-luminosity diagram is that both radio-quiet and radio-loud clumps possess a similar range of sub-mm colours. This implies that all the clumps in the diagram possess a similar range of temperatures and dust emissivities. The sub-mm properties of the radio-quiet clumps closely resemble those of the radio-loud clumps, suggesting that the clumps form a fairly homogenous population. It is intriguing to speculate that the main difference between the two is the presence or absence of an UC HII region as this naturally gives rise to the implication that the clumps are in different evolutionary states. However, the tendency for the radio-quiet clumps to

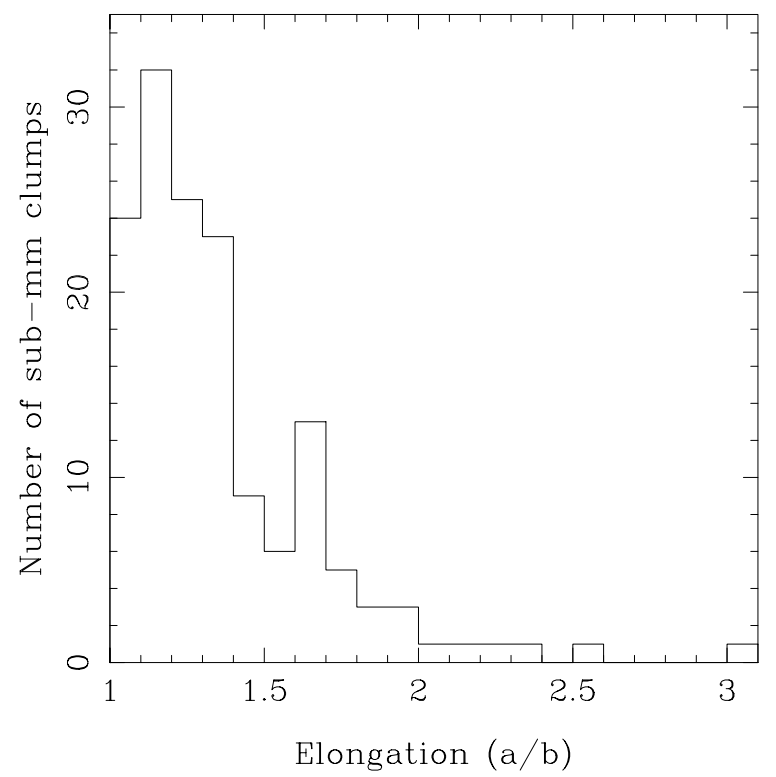

Fig. 5. Histogram of $850 \mu \mathrm{m}$ clump elongation.

exhibit generally lower distance-scaled $850 \mu \mathrm{m}$ fluxes implies that the radio-quiet clump population may also contain clumps that are forming lower-mass stars rather than high mass protostars in a pre-UC HII region phase. We will dwell further upon the nature of the radio-loud and radio-quiet clumps in Sects. 4.3 and 4.5 .

\subsubsection{Morphology}

We examined the morphology of the sub-mm clumps detected in the survey by measuring their half-power diameters along both major and minor axes. The major and minor axes of the clumps were initially estimated by Gaussian fits. It was found during the fitting procedure that a significant fraction of the clumps could not be accurately fitted by Gaussians and so for consistency all major and minor axes were directly estimated from the $50 \%$ contours of each clump. Due to the better signal-to-noise of the $850 \mu \mathrm{m}$ images we restrict the following discussion solely to the morphology of the clumps at this wavelength. The half-power diameters of the clumps are more sensitive to the distribution of the central regions of the clumps rather than the low-lying flux in their outer regions. To investigate the general extent of the sub-mm emission and compare this to the recent survey of candidate high-mass protostellar objects (HMPOs) of Williams et al. (2004) we also measured the $850 \mu \mathrm{m}$ flux $Y$-factor (the ratio of integrated to peak flux) for each clump in the survey.. We plot histograms of the clump elongation $(a / b)$, and $Y$-factor $\left(S_{\text {integrated }} / S_{\text {peak }}\right)$ in Figs. 5 and 6 respectively.

The majority of the sub-mm clumps display small elongations, with a median elongation of 1.4, suggesting a predominantly spherical or low axis-ratio population. This of course assumes that the clumps observed at $850 \mu \mathrm{m}$ represent single objects. This assumption seems to generally hold for the clumps in this survey: elongated clumps with high signal-to-noise $450 \mu \mathrm{m}$ data remain as elongated single structures in the higher resolution $450 \mu \mathrm{m}$ images (e.g. G33.13-0.09 in panel 43S) and there is no correlation of elongation with distance, as would be expected if the more distant clumps are unresolved linear chains or ridges.

We do not see any differences between the morphology of radio-loud or radio-quiet clumps, histograms of the elongation 


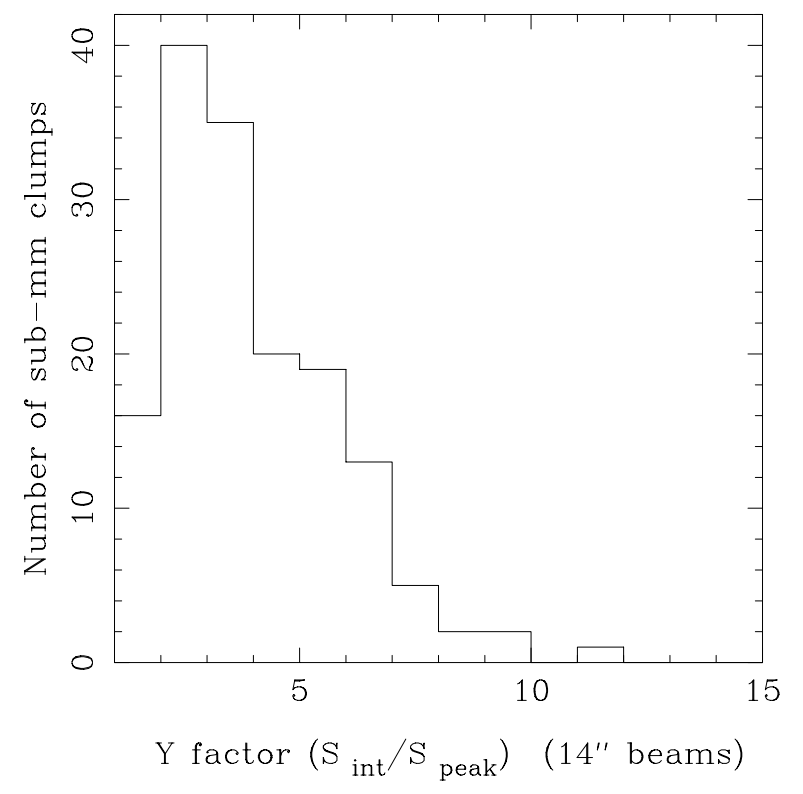

Fig. 6. Histogram of $850 \mu$ m clump $Y$ factor (integrated flux divided by peak flux). For an unresolved source the $Y$-factor is obviously equal to one.

of each population resemble each other closely and each sample possesses a similar median elongation (1.3 and 1.5 for radioloud and radio-quiet respectively). The radio-quiet clumps are marginally larger than the radio-loud clumps, with median major and minor axes of $30^{\prime \prime}$ and $19^{\prime \prime}$ for radio-quiet clumps as compared to $24^{\prime \prime}$ and $19^{\prime \prime}$ for the radio-loud clumps respectively.

The $Y$-factors of the sample as a whole (plotted in Fig. 6) follow a distribution that is peaked at a value of $\sim 3$. As in the histograms of elongation there is little difference between the histograms and median values of the $Y$-factor of radio-loud and radio-quiet clumps. The median value of the $Y$-factor for all sub-mm clumps detected in the survey is close to the value for candidate HMPOs observed by Williams et al. (2004). This may indicate that, as the clumps span a range in distance from $1-14 \mathrm{kpc}$, they could possess a scale-free envelope structure as suggested for candidate HMPOs by Williams et al. (2004). As mentioned previously we do not see any evidence that the objects in our study fragment at higher resolution, unlike the objects of Williams et al. (2004). However absence of evidence is not evidence of absence and caution must be applied to the hypothesis that the sub-mm clumps possess a scale-free envelope structure. Nevertheless we may at least draw the qualitative conclusion that, like the candidate HMPOs of Williams et al. (2004), a significant fraction of the mass of the clumps lies outside the central beam. If the radio-loud clumps in our survey represent embedded UC HII regions which are more evolved than the candidate HMPOs of Williams et al. (2004) then we may also conclude that the fraction of the mass in the outer region of the clump compared to that in the central beam does not evolve appreciably between the HMPO and UC HII stages.

\subsubsection{Clustering properties}

A substantial number of UC HII regions from the survey display emission from more than one sub-mm clump in the SCUBA field of view (for example G11.94-0.62 in panel 13L, G29.96-0.02 in panel 37L and G79.30+0.28 in panel 59L). Almost half of the SCUBA fields that were imaged during the survey $(44 / 105)$ are associated with two or more sub-mm clumps. Many of these "neighbouring" clumps have not been observed in suitable kinematic distance tracers and so it is not possible to determine whether these clumps are located in the same molecular cloud complex, or are a chance alignment of objects at different distances. Nevertheless the data that are so far available for individual cases (e.g. G34.26+0.15, Hunter et al. 1998; G10.30-0.15 and G29.96-0.02, Thompson et al. in preparation and G30.78-0.02, Motte et al. 2003) suggests that a number of the multiple clumps are clusters of objects found within the same molecular cloud complex. Several of the neighbouring sub-mm clumps are associated with star formation tracers: either UC HII regions (e.g. G28.29-0.36 in panel 36L) or $\mathrm{H}_{2} \mathrm{O}$ masers (e.g. G11.94-0.62 in panel 13L; Hofner \& Churchwell 1996), although a considerable number are radio-quiet. For an assumed distance of $5 \mathrm{kpc}$ each $2^{\prime}$ diameter SCUBA fields represents a $\sim 3$ pc diameter region, similar to the parsec-scale condensations within Giant Molecular Clouds (Blitz 1993). There is thus the important implication that these "neighbouring" submm clumps may represent other sites of star formation within the same molecular cloud complex containing the recently-formed YSO(s) exciting the UC HII region.

We quantify the likelihood of finding "neighbouring" clumps within a SCUBA field using the companion clump fraction (CCF), a statistic more often used to investigate the multiplicity of stars, but which has been used to investigate the multiplicity of candidate HMPOs (Williams et al. 2004). The $C C F$ gives a measure of the average number of companions per sub-mm clump and is given by the formula:

$C C F=\frac{B+2 T+3 Q}{S+B+T+Q}$,

where $S, B, T$ and $Q$ are the number of single, double (binary), triple and quadruple clumps in our survey, respectively. We find a $C C F$ for the sub-mm clumps detected in our survey of $0.90 \pm 0.07$, where the error was determined from simple Poisson counting statistics. This value is moderately higher than that reported by Williams et al. (2004) and, as the two surveys are at similar depths and of objects at similar distances, more than likely reflects the initial selection criteria of the sample upon which the Williams et al. study is based (Sridharan et al. 2002). The Sridharan et al. (2002) sample were selected to be isolated, in that the sources were non-detections in large-beam radio surveys and thus are not associated with UC HII regions nearer than a few arcminutes. Our UC HII region survey sample does not possess such selection criteria, which is reflected in the higher $C C F$ that we determine for the sub-mm clumps in our sample. As we did not select our sample on the grounds of isolation it can be argued that the higher $C C F$ is more representative of the multiplicity of sub-mm clumps in massive star-forming regions than the Williams et al. result. We have shown that it is highly likely for the sub-mm clumps in massive star-forming regions to possess at least one near neighbour (within a projected distance of a few pc). This suggests that massive YSOs or UC HII regions are ideal places to search for nearby, possibly less evolved companions and that the environments of UC HII regions are clustered active sites of massive star formation.

We also determined the surface density of the sub-mm clumps using the mean surface density of companions (MSDC) technique outlined in Williams et al. (2004), so that we could compare the surface density of clumps found toward more evolved UC HII regions to that measured for the relatively more isolated and potentially less evolved candidate HMPO clumps. 


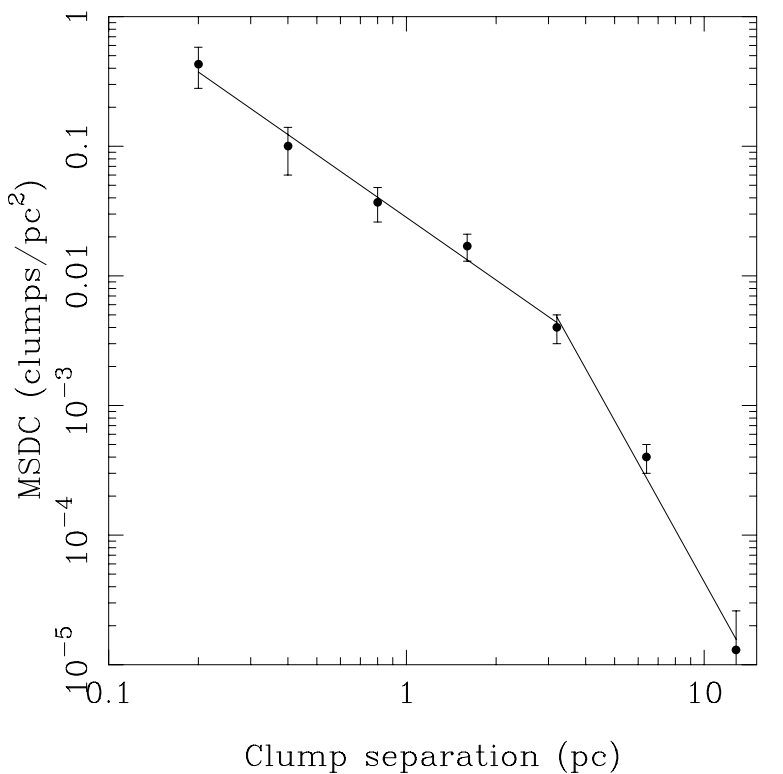

Fig. 7. The mean surface density of companions for all the $850 \mu \mathrm{m}$ clumps detected in the survey. Error bars are determined from simple Poisson counting statistics. Solid lines indicate linear least-squared fits to the first 5 and last 3 data points respectively.

We determined the MSDC by measuring the linear distance between pairs of clumps in the SCUBA images and scaling the number of linear distance pairs in annuli of fixed radius by the area of the distance annulus to obtain a value for the surface density. Clumps located in the same SCUBA field were assumed to lie at the same heliocentric distance. We present a plot of surface density versus linear separation in Fig. 7.

Figure 7 shows that the MSDC of the sub-mm clumps follows two power laws, with a break at $\sim 3 \mathrm{pc}$. At small linear separations the index of the power law is -1.6 , close to the value of -1.7 found for candidate HMPO clumps by Williams et al. (2004). But for linear separations over 3 pc the MSDC declines steeply with a power law index of -4.1 . The underlying cause behind this break is unclear; it is possibly due to incomplete sampling at large linear separations, but as the numbers of distance pairs in each distance bin are relatively constant apart from the very largest value we do not feel that this is a likely explananation. The decline in surface density over a radius of 3 pc may reflect a natural size scale for the extent of massive star-forming clusters in molecular clouds. If this is the case, one would also expect to see a similar effect in the candidate HMPO sub-mm clumps, particularly as the selection criteria of the HMPO sample select against radio-loud companions within several arcminutes (or several pc at the typical heliocentric distance of the HMPOs). This effect is not seen within the Williams et al. (2004) sample, whose surface density is consistent with a single unbroken power law of index -1.7 .

Our sample contains objects at a wide range of heliocentric distances (and thus a large range of linear distance pairs) and is limited at both small linear distances by the angular resolution of the JCMT and at large linear distances by the limited field of view of SCUBA. Our flux-limited, rather than mass-limited, sample may also cause a bias towards detecting bright, relatively nearby clumps at large linear separations or may underestimate the number of faint companion clumps. It is thus possible that the break in the power law is caused by a selection bias in our sample to particular ranges of linear distances. However, our sample of clumps observed towards UC HII regions is very similar to that observed by Williams et al. (2004) in terms of heliocentric distance and both surveys have a corresponding $850 \mu \mathrm{m}$ flux limit. We thus think it unlikely that the different MSDC distributions are caused by underlying selection biases in each sample. Further wide-field observations of a large sample of massive star-forming regions are required to more accurately the determine surface density distribution of sub-mm clumps and investigate whether the apparent steep decline in surface density at large separations is indicative of a natural size scale for regions of massive star formation.

\subsection{The likely nature of radio-loud sub-mm clumps}

Here we examine those sub-mm clumps associated with ultracompact radio sources (the radio-loud clumps) in order to determine whether their observed properties are consistent with massive clumps containing embedded UC HII regions. As we have already demonstrated, those ultracompact radio sources that are not associated with sub-mm emission (sub-mm quiet objects) are unlikely to be young embedded UC HII regions unless they lie at considerable heliocentric distance $(\geq 10 \mathrm{kpc})$. So, do the radioloud sub-mm clumps fit the standard picture of young UC HII regions that are still embedded within their natal molecular cloud cores?

The standard model of UC HII regions as discussed by e.g. Crowther \& Conti (2003) or Churchwell (2002) is of a small and young HII region photoionised by one or more high-mass stars and surrounded by a massive optically thick cocoon of dust and gas. Clearly the bright sub-mm clumps observed in our survey fit the picture of massive envelopes of dust and gas with sufficient dust to be optically thick to short-wavelength emission, but is the sub-mm emission consistent with being reprocessed radiation from the embedded star(s) responsible for exciting UC HII regions? If this were the case then a clear relation ought to exist between the sub-mm luminosity of the clumps (which arises from the reprocessed stellar luminosity) and the $\mathrm{cm}$-wave radio emission of the associated UC HII regions (which depends upon the UV luminosity of the stars). This relation has been shown to exist for a small number of UC HII regions from the WC89a and KCW94 catalogues by Crowther \& Conti (2003) using VLA cm-wave and IRAS $100 \mu$ m flux data. Crowther \& Conti's approach was unfortunately limited by confusion within the large $100 \mu \mathrm{m}$ beam of IRAS. Using our SCUBA images we can almost entirely remove these confusion issues and considerably enlarge the sample of UC HII regions over that of Crowther \& Conti (2003).

In Fig. 8 we present plots of the $850 \mu \mathrm{m} / \mathrm{cm}$-wave flux ratio against the number of Lyman continuum photons $\left(N_{\text {Lyc }}\right)$ for each radio-loud clump detected in our survey whose heliocentric distance is both known and unaffected by a near/far kinematic ambiguity. Lyman fluxes were calculated from the integrated radio fluxes of ultracompact radio sources associated with the sub-mm clumps as published in WC89a, KCW94, Becker et al. 1994 or Giveon et al. 2005 using the standard equation relating free-free continuum brightness to the Lyman continuum flux (e.g. Carpenter et al. 1990) and assuming that each radio source represents an UC HII region. Where multiple ultracompact radio sources were found to be associated with a single submm clump, the flux of each was summed to provide a total radio flux before evaluating the Lyman continuum flux. Diagrams are plotted for both 2 and $6 \mathrm{~cm}$ data, as many of the objects observed in the WC89a survey do not possess $6 \mathrm{~cm}$ data.

Caution must however be applied to the comparison of the derived Lyman flux from each VLA survey. The VLA surveys of 

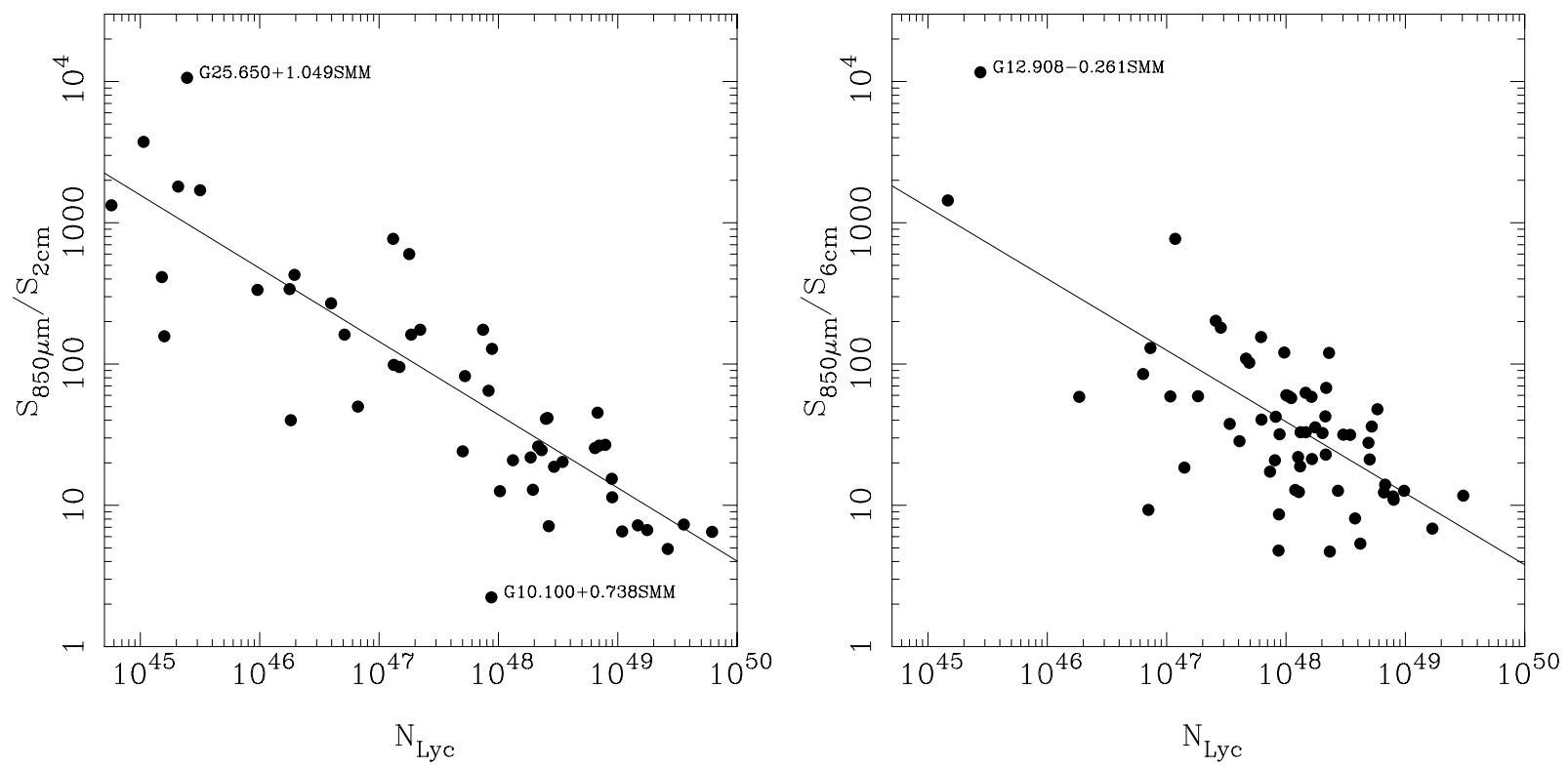

Fig. 8. Comparison between the radio to sub-mm ratio (left: $850 \mu \mathrm{m}$ vs. $2 \mathrm{~cm}$; right: $850 \mu \mathrm{m}$ vs. $6 \mathrm{~cm}$ ) and the Lyman photon flux inferred from the radio free-free emission of the UC HII regions. Solid lines represent linear least-squared fits to the data. Particular outliers to the fits are indicated and are discussed in the text.

WC89a, KCW94, Becker et al. (1994) and Giveon et al. (2005) were taken at different wavelengths and so all possess different selection effects, flux limits, synthesised beam sizes and sensitivity to extended structures. The $6 \mathrm{~cm}$ fluxes are mostly drawn from the sample of WC89a which was based on the single-dish point sources from Wink et al. (1982) with a $6 \mathrm{~cm}$ sensitivity of $\sim 0.5 \mathrm{Jy}$ and is therefore intrinsically biased against weak $6 \mathrm{~cm}$ emitters. The IRAS-selected sample of KCW94 which appears in the $2 \mathrm{~cm}$ plot (Fig. 8a) is however only limited by the sensitivity of their VLA observations. This explains the lack of low $N_{\text {Lyc }}$ sources at $6 \mathrm{~cm}$ in Fig. 8.

It is also possible that as the shorter wavelength data are less sensitive to extended structure (for the same $u v$ coverage and array configuration) the Lyman flux calculated from fluxes measured at this wavelength may be lower than that calculated from longer-wavelength data. We illustrate this effect in Fig. 9, where the $850 \mu \mathrm{m} / 2 \mathrm{~cm}$ flux ratio is plotted against distance. As can be seen in Fig. 9 there is a trend towards higher flux ratio values at smaller distances. Partly this is due to selection against faint radio sources at large distances, but this effect may also be caused by selection against nearby (i.e. within $\sim 2 \mathrm{kpc}$ ) UC HII regions with low flux ratios (and high $N_{\text {Lyc }}$ ) or that the $2 \mathrm{~cm}$ fluxes of nearby UC HII regions are underestimated due to the extended flux sensitivity issue described earlier.

We caution against overinterpretation of Fig. 8 but note that Fig. 9 reveals that the bias toward high values of $S_{850 \mu \mathrm{m}} / S_{2 \mathrm{~cm}}$ only becomes marked for UC HII regions with $S_{850 \mu \mathrm{m}} / S_{2 \mathrm{~cm}} \gtrsim$ 300 or $N_{\text {Lyc }} \lesssim 5 \times 10^{46} \mathrm{~s}^{-1}$. It can thus be seen that the correlation between the sub-mm/radio flux ratio seen in Fig. 8 holds for all except low values of $N_{\text {Lyc }}$ or high values of the sub-mm/cm-wave flux ratio.

Bearing in mind the caveats discussed in the previous paragraph, both plots in Fig. 8 show a clear linear relation between the $850 \mu \mathrm{m} /$ radio flux ratio and the Lyman continuum flux of the embedded UC HII regions. This linear relationship extends over more than four orders of magnitude in the number of Lyman photons $\left(N_{\text {Lyc }}\right)$ and almost three orders of magnitude in the $850 \mu \mathrm{m} /$ radio flux ratio. There are fewer objects with low Lyman

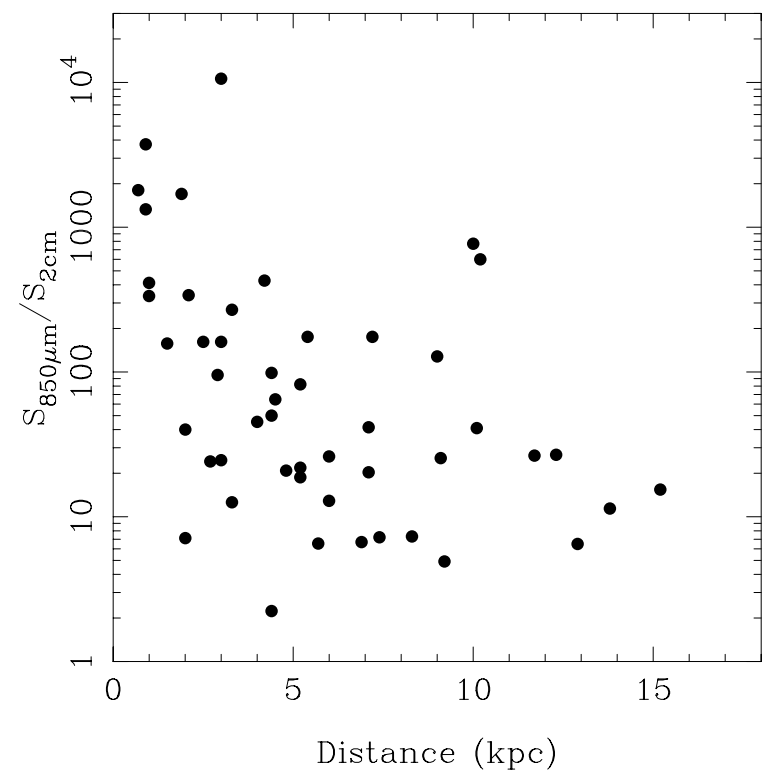

Fig. 9. The $2 \mathrm{~cm}$ to $850 \mu \mathrm{m}$ flux ratio of UC HII regions as a function of distance. Note the trend toward higher values of $S_{850 \mu \mathrm{m}} / S_{2 \mathrm{~cm}}$ at small distances which may indicate that the $2 \mathrm{~cm}$ fluxes of nearby UC HII regions are underestimated due to their over-resolution by the VLA.

flux in the $6 \mathrm{~cm}$ plot and the scatter is larger at $6 \mathrm{~cm}$ as compared to the $2 \mathrm{~cm}$ plot, but in general both plots agree closely. Figure 8 also shows that we do not see objects with either low $N_{\text {Lyc }}$ and low $850 \mu \mathrm{m}$ flux or high $N_{\text {Lyc }}$ and high $850 \mu \mathrm{m}$ flux. This is unlikely to be caused by any of the selection effects discussed earlier, as both the VLA surveys and our own SCUBA survey have sufficient sensitivity to detect these types of objects (if they exist) in these regions of the plot, either at low $N_{\text {Lyc }}$ or at low $850 \mu \mathrm{m}$ flux. A power-law fit to the data in both diagrams (following the form $y=A 10^{b x}$ ) yields values for $A$ of $10^{26.51 \pm 2.01}$ and $10^{25.88 \pm 3.32}$ at 2 and $6 \mathrm{~cm}$ respectively; and $b$ of $-0.52 \pm 0.04$ and $-0.51 \pm 0.07$, with straight-line fit coefficients of 0.9 and 0.7 for 2 and $6 \mathrm{~cm}$ respectively. 
Figure 8 clearly shows that the radio-loud sub-mm clumps in our survey are in excellent agreement with the standard model of embedded UC HII regions and that the clumps are predominantly heated by their embedded massive stars. Hereafter in this paper we will refer to these ultracompact radio components as UC HII regions. The scatter in both diagrams is likely to be caused by the selection effects mentioned earlier, the absorption of Lyman continuum photons within the UC HII regions by dust (which would move points leftwards and upwards) or by the presence of stars of lower mass that contribute to the total IR flux but do not contribute to the Lyman flux (which would move points vertically upwards). We indicate two specific clumps in particular whose outlying positions in Fig. 8 may be due to these effects (G25.650+1.049SMM and G12.908-0.261SMM). We also indicate the object G10.100+0.738SMM in Fig. 8, whose outlying position at the bottom of the diagram is unlikely to be caused by either selection effects, Lyman photon absorption or the presence of lower-mass stars. This object is almost certainly a misclassified UC HII region. Walsh et al. (2003) classify this object as a planetary nebula, noting that the position of the sub-mm clump coincides with the position of the well-known planetary nebula NGC 6537. The position of G10.100+0.738SMM in Fig. 8 confirms this hypothesis.

\subsection{Positional association of UC HII regions and methanol masers with sub-mm clumps}

The positional association of ultracompact radio components and sub-mm clumps has already been discussed in Sect. 4.2 where we identified both radio-loud and radio-quiet clumps; and in Sect. 4.3 where we showed that "sub-mm loud" radio components are strongly consistent with being UC HII regions. The angular resolution of our SCUBA images not only identifies whether the UC HII regions in our survey are associated with sub-mm clumps, but also serves to study the (projected) position of the $\mathrm{cm}$-wave radio emission with respect to the peak of their associated sub-mm clump. The location of UC HII regions within their embedding clumps potentially allows us to investigate the characteristics of the birthplaces of the massive OB stars that excite the UC HII regions. For example, are the massive stars born within dense central cores within the clumps or are their birthsites offset from the density peak of the clump as suggested by the hierarchical model of Kim \& Koo (2001)?

In Fig. 10 we present a histogram of the angular distance between UC HII regions and the peak position of the closest submm clump. For comparison we also plot a similar histogram of the angular distance between methanol masers and sub-mm clumps from Walsh et al. (2003), which is shown as a shaded histogram. As in previous sections we have restricted our analysis to the higher signal-to-noise $850 \mu \mathrm{m}$ data. As can be seen in Fig. 10 the two angular distance distributions are markedly different; methanol masers follow a very tightly peaked distribution whereas the UC HII regions show a dip at small distances with a much broader overall distribution.

The tight correlation of masers with sub-mm peaks is evidenced by Walsh et al's detection statistics; all but one of the 84 masers surveyed was associated with sub-mm emission and $83 \%$ were found within $5^{\prime \prime}$ of a sub-mm peak (Walsh et al. 2003). Figure 10 shows that the FWHM of the methanol maser angular distance distribution is $\sim 5^{\prime \prime}$. Methanol masers are thus predominantly found toward the peak positions of sub-mm clumps, which suggests that the masers trace deeply embedded star formation at or very near (at a median distance of $5 \mathrm{kpc}$, $5^{\prime \prime}$ corresponds to a projected linear distance of $0.1 \mathrm{pc}$ ) to the

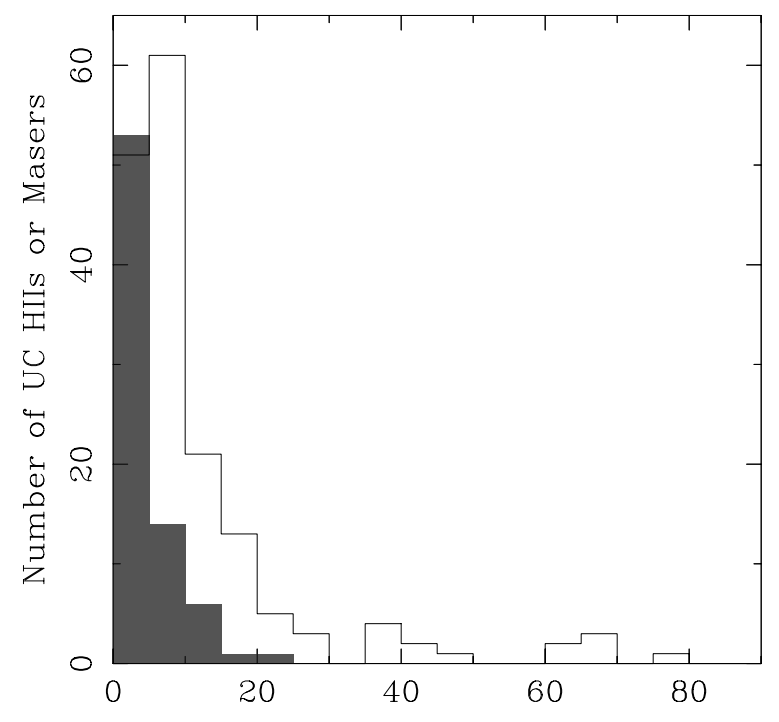

Angular distance to nearest sub-mm peak (arcsec)

Fig. 10. Histogram of the angular distance of the UC HII regions to the nearest sub-mm peak (unfilled histogram) plotted over a histogram of the angular distance of the methanol masers from Walsh et al. 2003 to their nearest sub-mm peak (shaded histogram). Note the difference in the two populations in terms of the central peak and the width of the distribution.

centre of the dense dusty clumps traced by the sub-mm emission. The dip in the ultracompact radio component distribution shows that the majority of UC HII regions are found approximately twice as far from the sub-mm peak positions as methanol masers, i.e. UC HII regions are more likely to be offset from the centre of their dense dusty embedding clumps. The width of the ultracompact radio distribution is also twice as large as that of the methanol maser distribution.

The observed dip in the UC HII region distribution is small compared to the FWHM of the JCMT beam, but is twice as large as the typical pointing accuracy that was achieved during the observations $\left(\leq 5^{\prime \prime}\right)$. We examined our data carefully to exclude systematic errors in the pointing by comparing histograms for sources that were obtained on different nights. These histograms all displayed a similar distribution, indicating that systematic pointing effects are not the cause of the observed dip. We also note that the Walsh et al. (2003) data do not display a similar distribution, even though they were obtained under similar conditions and with the same instrument. Finally, exactly the same distribution was observed from independent data: the angular distance between the UC HII positions determined by Walsh et al. (1998) as part of their ATCA maser survey and the sub-mm peaks observed by Walsh et al. (2003). We thus conclude that the difference between the methanol maser and ultracompact radio component angular distance distributions is likely to be a real effect. A similar tight correlation between the positions of methanol masers and their associated sub-mm clumps is reported by Beuther et al. (2002), who found an average linear separation of just $0.03 \mathrm{pc}$ between the masers and their associated millimetre continuum peaks

Why are methanol masers and ultracompact HII regions found at predominantly different distances from the peak of their associated sub-mm clumps? One clue may lie in the different evolutionary states that are traced by methanol masers 
and UC HII regions. Simple models of maser formation (Walsh et al. 1998; Codella \& Moscadelli 2000) suggest that methanol masers are initially formed in the dense molecular environments of luminous pre-ZAMS high-mass stars, radiatively pumped by the strong mid-IR emission from the deeply embedded stars, then are destroyed as an UC HII region forms and expands around the young high-mass star. If this is the case then methanol masers should trace younger objects than UC HII regions.

Discounting projection effects, which should be negligible in our large sample, we identify three possibilities to explain the relative absence of UC HII regions in the centres of sub-mm clumps:

1. UC HII regions in the densest regions of sub-mm clumps are quenched for some fraction $(\sim 1 / 2)$ of their lifetime, perhaps by accretion;

2. UC HII regions are preferentially formed away from the dense central regions of the sub-mm clumps;

3. UC HII regions or their direct precursors clear enough gas and dust to destroy the submm peaks in which they formed, shifting the observed SCUBA peak away from the UC HII region.

The former two hypotheses explain the absence of UC HII regions from the dense central cores of sub-mm clumps in terms of reducing the number of observable UC HII regions at high densities; either quenching shortens the lifetime of the UC HII phase, or UC HII regions are mostly formed some distance from the local density peak, as suggested by the Kim \& Koo (2001) hierarchical model. However, neither of these hypotheses can explain the difference in distribution between methanol masers and ultracompact radio components. If methanol masers are indeed the precursors to UC HII regions then they should share the same angular distance distributions. Reducing the number of observed UC HII regions at the sub-mm peak positions does not resolve the issue of why the overall angular distance distribution of UC HII regions is twice as broad as that of methanol masers, nor why methanol masers are tightly correlated with the sub-mm peak positions.

The clearing hypothesis may provide a better answer. As UC HII regions expand they begin to clear away their surrounding gas and dust, ultimately resulting in the eventual destruction of the molecular cloud in which the high mass star was born. As the UC HII expands in its early stages the first region to be cleared should be the dense dusty core within which the high mass star was born. These cores are typically $\leq 0.1 \mathrm{pc}$ in diameter (Kurtz et al. 2000) and would hence be unresolved by SCUBA. As this core is destroyed the peak position of the larger clump observed by SCUBA (which does not resolve the individual cores comprising the clump) would shift to the next most dense core (i.e. next highest column density) within the clump, effectively changing the UC HII/sub-mm peak distance. This effect may be complicated by the fact that the UC HII region will still be surrounded by a shell of warm dust, but as long as the column density of the next most dense core is sufficiently higher than that of the shell surrounding the UC HII, then the observed sub-mm peak position will be offset from that of the UC HII. Hence, the methanol maser phase of high-mass star formation is tightly correlated with the peak of the SCUBA submm clump, then the apparent peak of the clump shifts as the UC HII develops and destroys the dense core within which it is embedded. The width of the dip in the UC HII region distribution yields an estimate of the clearing radius, i.e. $5^{\prime \prime}$ or $0.1 \mathrm{pc}$ at $5 \mathrm{kpc}$.
The difficulty with this hypothesis rests upon whether the relatively small diameter UC HII regions can clear dust on these scales. The diameters of UC HII regions measured by WC89 and KCW94 are typically 2-4", but more recent observational results show that UC HII can in fact possess much larger ionised regions on arcminute scales (e.g. Kurtz et al. 1999; Kim \& Koo 2001; Ellingsen et al. 2005). The dust is more difficult to clear than gas, but the dust sublimation boundary of UC HII regions can be as much as $0.3-0.4$ times the ionised gas radius initially and throughout the expansion phase (Inoue 2002; Arthur et al. 2004). In addition, the analysis of Franco et al. (2000) suggests that in strongly-peaked density distributions $\left(\geq r^{-3 / 2}\right)$ the ionisation front of the UC HII region undergoes a runaway expansion that expands at $\sim 0.5 \mathrm{pc} / \mathrm{Myr}$ or more. From modelling of clumps associated with UC HII regions we know that their density distributions are steeper than $r^{-3 / 2}$ (Hatchell \& van der Tak 2003) and so it is likely that the UC HII regions in these steep density distributions rapidly destroy and clear their dense birthplaces, shifting the observed SCUBA sub$\mathrm{mm}$ peak to the next highest column density peak within the clump.

Corroborating observational evidence to this hypothesis may be found in high-resolution interferometric studies of UC HII regions and their molecular environment. The edges of UC HII regions are often truncated by the presence of nearby dense hot molecular cores, e.g. the well-known UC HII regions G29.96-0.02 and G9.62+0.19 (Cesaroni et al. 1994). Confirmation of the small effect uncovered in our SCUBA imaging using higher resolution millimetre or sub-millimetre interferometry is a priority to determine whether clearing by the UC HII regions is indeed responsible.

\subsection{Radio-quiet sub-mm clumps: are they precursors to UC $H$ II regions?}

We have already shown (in Sect. 4.3) that the radio-loud clumps are strongly consistent with the standard model of embedded UC HII regions, but what is the likely nature of the radio-quiet clumps? A cursory inspection of Figs. 1 and 2 and Table 2 does not reveal any immediate differences between the fluxes or morphology of radio-quiet and radio-loud clumps, with perhaps the only difference that the radio-quiet clumps possess on average lower peak and integrated sub-mm fluxes than the radio-louds. There are six main possibilities for the nature of the radio-quiet clumps:

i) the clumps contain embedded UC HII regions that are too compact and/or optically thick to have been detected in the VLA surveys;

ii) conversely, the radio-quiet clumps may contain diffuse HII regions that are extended on scales larger than that to which the VLA surveys were sensitive;

iii) the clumps are not gravitationally bound and will thus not form stars;

iv) the clumps are star-forming, but are forming low- or intermediate-mass stars that do not produce HII regions rather than $\mathrm{OB}$ stars;

v) the clumps are star-forming and have not yet formed massive OB stars;

vi) the clumps are star-forming and contain dusty UC HII regions in which absorption from the dust reduces the UV photon flux and hence the free-free emission below the detection levels of the VLA surveys. 
We briefly examine each of these possibilities in turn, to try to ascertain the likely nature of the radio-quiet clumps.

\section{i) Undetected ultracompact $\mathrm{H}$ II regions:}

With the current data it is not possible to completely rule out scenarios in which the radio-quiet clumps contain embedded UC HII regions that were not detected in the VLA surveys of WC89a and KCW94. From the radio flux upper limits of WC89a and KCW94 (typically $0.4-1 \mathrm{mJy} /$ beam at $2 \mathrm{~cm}$ ) we may evaluate a lower limit to the radius of a detectable optically thick UC HII region using Eqs. (4) and (5) from Molinari et al. (2000). Assuming an upper limit to the $2 \mathrm{~cm}$ flux of $1 \mathrm{mJy}$ and a synthesised beam $F W H M$ of 0 .'5, we derive a lower limit to the detectable radius of an UC HII of $r_{\mathrm{u}} \geq 6.7 \times 10^{-8} d \mathrm{pc}$, where $d$ is the distance to the UC HII region in pc.

Unfortunately, the distances to many of the radio-quiet clumps are highly uncertain, as there is often no kinematic tracer (radio-recombination line or maser) associated with the clump. If we assume that the radio-quiet clumps are at the same typical distance as those clumps containing UC HII regions then from Table 1, we expect the upper bound on the distance to be $\sim 14 \mathrm{kpc}$, at which distance the VLA surveys are only sensitive to UC HII regions $\geq 9 \times 10^{-4} \mathrm{pc}$. For a more fiducial distance of $5 \mathrm{kpc}$ this upper limit falls to $3 \times 10^{-4} \mathrm{pc}$. In comparison, the initial Strömgren radius for an O6 star surrounded by a pure hydrogen nebula of density $n=2 \times 10^{7} \mathrm{~cm}^{-3}$ is $\sim 10^{-3}$ pc (de Pree et al. 1995).

Assuming all the radio-quiet clumps are populated by UC HII regions at or above their dust-free Strömgen radius, the $2 \mathrm{~cm}$ upper limits rule out essentially all $\mathrm{O}$ stars and B0 stars in all but the furthest $20 \%$ of sources. Later-type B stars are not ruled out: B0.5 stars in all but the $20 \%$ closest sources, and latertype B stars at all survey distances, would fall below the $2 \mathrm{~cm}$ flux limit. Of course, the time to produce a Strömgren sphere is very short and UC HII regions subsequently expand, making them more observable, and although the expansion is inhibited over that expected by simple pressure arguments, WC89a see few sources at their initial Strömgren radii. On the other hand, both dust absorption and higher densities reduce the detectability by reducing the Strömgren radius. In the case of $90 \% \mathrm{UV}$ absorption by dust, we would also fail to detect 09.5 stars in the more distant half of the sample. The closer the radio-quiet clump, the higher the probability of containing no UC HII or a very young and therefore compact UC HII. We thus conclude that it is possible for the radio-quiet clumps to contain late $\mathrm{O}$ or early B-type stars. A wider investigation of the kinematic distances of the radio-quiet clumps is required to reveal their potential to host massive stars or undetected UC HII regions.

\section{ii) Extended $\mathrm{H}$ II regions:}

Due to the fact that interferometers such as the VLA screen out extended radio emission, the converse that some HII regions are too extended to be detected is also true. The largest angular scales that the WC89a and KCW94 surveys were sensitive to is $\sim 10^{\prime \prime}$ at $2 \mathrm{~cm}$. Because both of these surveys were carried out in snapshot mode, the sparse $u v$ coverage of the resulting data means that the largest angular scale should be considered to be an upper limit. HII regions larger than $10^{\prime \prime}$ with a smooth surface brightness distribution would not have been detected, although bright components of clumpy HII regions would possibly have been detected as a "cluster" of UC HII regions. This effect may be seen in some of the KCW94 UC HII regions, which merge to form a larger HII region in more compact VLA configuration images (Kurtz et al. 1999), or those clustered objects in the Giveon et al. (2005) catalogue which were discussed in more detail in Sect. 4.1. However, it is unlikely that all the radio-quiet clumps are associated with extended HII regions and in any case these objects inform us as to the prior massive star formation that has taken place in the region, not deeply embedded star-formation that may be currently taking place. These radio-quiet clumps fall into categories $v$ ) or $v i$ ) below.

\section{iii) Unbound clumps, and iv) Low mass star formation:}

The Jeans mass for a $50 \mathrm{~K}, n=10^{4} \mathrm{~cm}^{-3}$ molecular cloud is about $80 M_{\odot}$, which produces a $850 \mu \mathrm{m}$ flux of $\sim 1 \mathrm{Jy}$ at $5 \mathrm{kpc}$. On this basis, a few weak, nearby, radio-quiet clumps could be unbound (about $15 \%$ of the sample), but the remaining $85 \%$ fall above this limit.

It is more difficult to rule out the presence of low-mass star forming clusters in our sample, particularly for the less luminous objects. Two examples of low-mass star forming clusters are NGC 1333 and L1448, which were recently observed at $850 \mu \mathrm{m}$ as part of a survey of the Perseus molecular cloud (Hatchell et al. 2005). Both NGC 1333 and L1448 contain several hundred $M_{\odot}$ of dust and gas within a few $\mathrm{pc}^{2}$, and their resulting $850 \mu \mathrm{m}$ fluxes are of the order 200-300 Jy. This implies that clusters of this type could have been detected out to a distance of $\sim 5.5 \mathrm{kpc}$ in our survey (assuming a typical detection limit of $<1 \mathrm{Jy}$ at $850 \mu \mathrm{m}$ ). We thus conclude that the weaker radio-quiet clumps detected in our survey may be forming clusters of lowmass stars. It is difficult to point to specific examples as many of the radio-quiet clumps do not possess measured kinematic distances. Follow-up observations to determine the distance (and hence luminosity) of these objects are a priority.

iv) pre-UC H II massive protostars and v) young massive stars with dust-quenched UC H II:

Of all of these possibilities the last two are by far the most intriguing, as they imply that the radio-quiet clumps may contain a population of still-accreting massive stars with newly-formed UC HII, and the long sought-after massive protostellar precursors to UC HII regions.

With the current body of observational data it is not possible to completely determine the nature of the radio-quiet clumps. A full investigation of the SCUBA-detected clumps near UC HII, using molecular line observations to probe the temperatures, chemical composition, structure and dynamics of the cores, will be reported at a later date.

\section{Summary and conclusions}

We present the results of a sub-mm continuum imaging survey of UC HII regions, performed with the SCUBA bolometer array on the JCMT. A total of 105 IRAS sources from the UC HII region catalogues of Wood \& Churchwell (1989a) and Kurtz et al. (1994) were mapped at 450 and $850 \mu \mathrm{m}$ using SCUBA's jiggle-mapping mode. We detected 155 sub-mm clumps within the SCUBA images and identify three kinds of object within our survey: "sub-mm-quiet objects" which are ultracompact $\mathrm{cm}$-wave radio sources that are not associated with sub-mm 
emission; "radio-loud" sub-mm clumps which are associated with ultracompact cm-wave sources; and "radio-quiet" sub-mm clumps which are not associated with detectable cm-wave emission. A number of IRAS point sources (14 in total) were found to exhibit no emission at $850 \mu \mathrm{m}$ greater than $0.1-0.3 \mathrm{Jy} / \mathrm{beam}$. We draw the following conclusions from our survey:

a) The sub-mm-quiet ultracompact radio components from the WC89a, KCW94, Becker et al. (1990) and Giveon et al. (2005) surveys are unlikely to be true embedded UC HII regions unless they are located at distances greater than $10 \mathrm{kpc}$. We preclude embedding clump masses in excess of a few solar masses for objects at distance less than $10 \mathrm{kpc}$. We suggest that the sub-mm quiet objects are likely either planetary nebulae or bright knots embedded within larger HII regions.

b) The sub-mm clumps as a whole possess similar sub-mm colours and distance-scaled fluxes, which suggests that they are drawn from similar populations and have similar masses, luminosities and temperatures. There is a tendency for radio-quiet sub-mm clumps to exhibit lower distance-scaled fluxes which may indicate that these objects are, on average, of lower luminosity than radio-loud clumps.

c) The sub-mm clumps display small elongations with a median value of 1.4 , which suggests a predominantly spherical or low axis ratio population. The ratio of peak to source-integrated flux follows a similar distribution to the High-Mass Protostellar Object (HMPO) candidates observed by Williams et al. (2004), which may indicate that the fraction of the mass in the outer regions of the clumps does not evolve significantly between the HMPO and UC HII region phases. We also find that there is little difference between the morphology of radio-quiet and radio-loud clumps.

d) The clumps are moderately clustered. Out of $1052^{\prime}$ diameter images we detected 155 clumps. The Companion Clump Fraction $(\mathrm{CCF})$ of the clumps is $0.90 \pm 0.07$, which is higher than the value of 0.65 found for HMPOs by Williams et al. (2004). We suggest that the difference is real, and the selection bias against nearby UC HIIs in the Williams et al. study results in a lower clump companion fraction.

e) The mean surface density of clump companions (MSDC) follows a broken power-law distribution, with a break at a clump separation of $3 \mathrm{pc}$. This may reflect a natural size scale for the extent of massive star-forming clusters in molecular clouds, although our sample of objects at a wide range of heliocentric distances and flux-limited data could introduce selection biases against distant or faint companions. Further wide-field observations of a more uniform distance selected sample are required to address this issue more rigorously.

f) Radio-loud clumps are shown to be strongly consistent with the standard model of embedded UC HII regions (e.g. Crowther \& Conti 2003; Churchwell 2002). The Lyman continuum fluxes are in excellent agreement with the $850 \mu \mathrm{m}$ to $\mathrm{cm}$-wave flux ratio, showing that the YSOs exciting the UC HII regions are responsible for heating the sub-mm clumps. We also identify one object within the survey whose $850 \mu \mathrm{m}$ flux is inconsistent with the standard model (G10.100+0.738SMM) and suggest that this sub-mm emission from this object originates from the planetary nebula NGC 6537.

g) We show that UC HII regions are predominantly found at an angular distance of $\geq 10^{\prime \prime}$ from the sub-mm peak of their associated sub-mm clump. The distribution of these angular distances is clearly different to that of methanol masers and their associated sub-mm clumps derived from the data of Walsh et al. (2003). We suggest that the most likely hypothesis to explain the differences in the two distributions is that UC HII regions efficiently clear their immediate surroundings of dense material, shifting the sub-mm peak observed by SCUBA to the next highest column density peak within the clump. We estimate a value for the clearing radius of $\sim 0.1 \mathrm{pc}$, for a median distance in our sample of $5 \mathrm{kpc}$.

h) We speculate upon the nature of the radio-quiet clumps and conclude that they are unlikely to contain embedded $\mathrm{O}$-stars. We cannot rule out the presence of embedded B-stars, particularly in the furthest objects. Only the closest $15 \%$ of radio-quiet clumps possess sub-mm fluxes that imply they are either gravitationally unbound or likely to be forming only low mass stars, and hence we conclude that the majority of the sample are massive and luminous enough to form intermediate or high-mass stars. We do not have sufficient data to conclude that the radio-quiet clumps are in a pre-UC HII region phase, but we argue that their characteristics are suggestive of such a stage. We will present a full investigation of these clumps at a later date.

Acknowledgements. The authors would like to thank all of the anonymous visiting observers and JCMT support staff who undertook the flexibly scheduled observations. We would also like to thank the referee for the many useful suggestions and careful reading of the paper. This research made use of data products from the Midcourse Space Experiment obtained from the NASA/ IPAC Infrared Science Archive, which is operated by the Jet Propulsion Laboratory, California Institute of Technology, under contract with the National Aeronautics and Space Administration.. MSX data processing was funded by the Ballistic Missile Defense Organization with additional support from NASA Office of Space Science. This research would not have been possible without the SIMBAD astronomical database service operated at CCDS, Strasbourg, France and the NASA Astrophysics Data System Bibliographic Services. JH is supported at Exeter by a PPARC AF.

\section{References}

Acord, J. M., Churchwell, E., \& Wood, D. O. S. 1998, ApJ, 495, L107 Araya, E., Hofner, P., Churchwell, E., \& Kurtz, S. 2002, ApJS, 138, 63 Archibald, E. N., Jenness, T., Holland, W. S., et al. 2002, MNRAS, 336, 1 Argon, A. L., Reid, M. J., \& Menten, K. M. 2000, ApJS, 129, 159 Arthur, S. J., Kurtz, S. E., Franco, J., \& Albarrán, M. Y. 2004, ApJ, 608, 282

Aspin, C., \& Geballe, T. R. 1992, A\&A, 266, 219

Bally, J., Reipurth, B., Walawender, J., \& Armond, T. 2002, AJ, 124, 2152 Baudry, A., Diamond, P. J., Booth, R. S., Graham, D., \& Walmsley, C. M. 1988, A\&A, 201, 105

Becker, R. H., White, R. L., McLean, B. J., Helfand, D. J., \& Zoonematkermani, S. 1990, ApJ, 358, 485

Becker, R. H., White, R. L., Helfand, D. J., \& Zoonematkermani, S. 1994, ApJS, 91, 347

Bertin, E., \& Arnouts, S. 1996, A\&AS, 117, 393

Beuther, H., Walsh, A., Schilke, P., et al. 2002, A\&A, 390, 289

Bica, E., Dutra, C. M., \& Barbuy, B. 2003, A\&A, 397, 177

Blitz, L. 1993, Protostars and Planets III, 125 (Tucson: Arizona Press), ed. Levy \& Lunine 
Blum, R. D., Conti, P. S., \& Damineli, A. 2000, AJ, 119, 1860

Brand, J., Cesaroni, R., Comoretto, G., et al. 2003, A\&A, 407, 573

Braz, M. A., \& Epchtein, N. 1983, A\&AS, 54, 167

Carpenter, J. M., Snell, R. L., \& Schloerb, F. P. 1990, ApJ, 362, 147

Carral, P., \& Welch, W. J. 1992, ApJ, 385, 244

Caswell, J. L., Vaile, R. A., Ellingsen, S. P., Whiteoak, J. B., \& Norris, R. P. 1995, MNRAS, 272, 96

Cesaroni, R., Churchwell, E., Hofner, P., Walmsley, C. M., \& Kurtz, S. 1994, A\&A, 288, 903

Churchwell, E., Walmsley, C. M., \& Cesaroni, R. 1990, A\&AS, 83, 119

Churchwell, E. 2002, ARA\&A, 40, 27

Clark, P. C., Bonnell, I. A., Zinnecker, H., \& Bate, M. R. 2005, MNRAS, 359, 809

Codella, C., Felli, M., Natale, V., Palagi, F., \& Palla, F. 1994, A\&A, 291, 261

Codella, C., \& Moscadelli, L. 2000, A\&A, 362, 723

Crowther, P. A., \& Conti, P. S. 2003, MNRAS, 343, 143

Currie, M. J., \& Berry, D. S. 2002, Starlink User Note 95, Starlink Project, CCLRC

Dartois, E., Gerin, M., \& d'Hendecourt, L. 2000, A\&A, 361, 1095

De Buizer, J. M., Watson, A. M., Radomski, J. T., Piña, R. K., \& Telesco, C. M. 2002, ApJ, 564, L101

Dent, W. F. R., Matthews, H. E., \& Ward-Thompson, D. 1998, MNRAS, 301, 1049

Depree, C. G., Goss, W. M., Palmer, P., \& Rubin, R. H. 1994, ApJ, 428, 670

de Pree, C. G., Rodriguez, L. F., \& Goss, W. M. 1995, Rev. Mex. Astron. Astrofis. 31,39

Economou, F., Jenness, T., Currie, M., et al. 2002, Starlink User Note 230, Starlink Project, CCLRC

Ellingsen, S. P., Shabala, S. S., \& Kurtz, S. E. 2005, MNRAS, 357, 1003

Feldt, M., Stecklum, B., Henning, T., et al. 1998, A\&A, 339, 759

Feldt, M., Stecklum, B., Henning, T., Launhardt, R., \& Hayward, T. L. 1999, A\&A, 346, 243

Fey, A. L., Gaume, R. A., Nedoluha, G. E., \& Claussen, M. J. 1994, ApJ, 435, 738

Fish, V. L., Reid, M. J., Wilner, D. J., \& Churchwell, E. 2003, ApJ, 587, 701

Fontani, F., Cesaroni, R., Caselli, P., \& Olmi, L. 2002, A\&A, 389, 603

Franco, J., Kurtz, S. E., García-Segura, G., \& Hofner, P. 2000, Ap\&SS, 272, 169

Garay, G., \& Lizano, S. 1999, PASP, 111, 1049

Garay, G., Moran, J. M., Rodriguez, L. F., \& Reid, M. J. 1998, ApJ, 492, 635

Garay, G., Faúndez, S., Mardones, D., et al. 2004, ApJ, 610, 313

Gaume, R. A., Fey, A. L., \& Claussen, M. J. 1994, ApJ, 432, 648

Ghosh, S. K., Iyengar, K. V. K., Karnik, A. D., et al. 2000, Bulletin of the Astronomical Society of India, 28, 515

Giveon, U., Becker, R. H., Helfand, D. J., \& White, R. L. 2005, AJ, 129, 348

Goedhart, S., van der Walt, D. J., \& Schutte, A. J. 2000, MNRAS, 315, 316

Gómez, J. F., Sargent, A. I., Torrelles, J. M., et al. 1999, ApJ, 514, 287

Gomez, Y., Rodriguez, L. F., Garay, G., \& Moran, J. M. 1991, ApJ, 377, 519

Gomez, Y., Rodriguez, L. F., \& Marti, J. 1995, ApJ, 453, 268

Gómez, Y., Rodríguez, L. F., \& Garay, G. 2000, ApJ, 531, 861

Gómez, Y., Rodríguez, L. F., \& Garay, G. 2002, ApJ, 571, 901

Gómez, Y., Rodríguez, L. F., Girart, J. M., Garay, G., \& Martí, J. 2003, ApJ, 597, 414

Green, A. J., Frail, D. A., Goss, W. M., \& Otrupcek, R. 1997, AJ, 114, 2058

Grim, R. J. A., Baas, F., Greenberg, J. M., Geballe, T. R., \& Schutte, W. 1991, A\&A, 243, 473

Hanson, M. M., Howarth, I. D., \& Conti, P. S. 1997, ApJ, 489, 698

Harju, J., Lehtinen, K., Booth, R. S., \& Zinchenko, I. 1998, A\&AS, 132, 211

Harvey, P. M., \& Forveille, T. 1988, A\&A, 197, L19

Hatchell, J., Thompson, M. A., Millar, T. J., \& MacDonald, G. H. 1998, A\&AS 133, 29

Hatchell, J., Fuller, G. A., Millar, T. J., Thompson, M. A., \& Macdonald, G. H. 2000, A\&A, 357, 637

Hatchell, J., \& van der Tak, F. F. S. 2003, A\&A, 409, 589

Hatchell, J., Richer, J. S., Fuller, G. A., et al. 2005, A\&A, 440, 151

Heaton, B. D., Matthews, N., Little, L. T., \& Dent, W. R. F. 1985, MNRAS, 217, 485

Hildebrand, R. H. 1983, QJRAS, 24, 267

Hill, T., Burton, M. G., Minier, V., et al. 2005, MNRAS, in press [arXiv:astro-ph/0506402]

Hofner, P., Kurtz, S., Churchwell, E., Walmsley, C. M., \& Cesaroni, R. 1994, ApJ, 429, L85

Hofner, P., \& Churchwell, E. 1996, A\&AS, 120, 283

Hofner, P., Kurtz, S., Churchwell, E., Walmsley, C. M., \& Cesaroni, R. 1996, ApJ, 460, 359

Hogerheijde, M. R., \& Sandell, G. 2000, ApJ, 534, 880
Holland, W. S., et al. 1999, MNRAS, 303, 659

Howard, E. M., Koerner, D. W., \& Pipher, J. L. 1997, ApJ, 477, 738

Hughes, V. A., \& Wouterloot, J. G. A. 1984, ApJ, 276, 204

Hughes, V. A. 2001, ApJ, 563, 919

Hunter, T. R., Benford, D. J., \& Serabyn, E. 1996, PASP, 108, 1042

Hunter, T. R., Phillips, T. G., \& Menten, K. M. 1997, ApJ, 478, 283

Hunter, T. R., Neugebauer, G., Benford, D. J., et al. 1998, ApJ, 493, L97

Hunter, T. R., Churchwell, E., Watson, C., et al. 2000, AJ, 119, 2711

Inoue, A. K. 2002, ApJ, 570, 688

Jaffe, D. T., Hildebrand, R. H., Keene, J., et al. 1984, ApJ, 281, 225

Jenness, T., \& Lightfoot, J. F. 2000, Starlink User Note 216, Starlink Project, CCLRC

Kerton, C. R., Martin, P. G., Johnstone, D., \& Ballantyne, D. R. 2001, ApJ, 552, 601

Kim, K., \& Koo, B. 2001, ApJ, 549, 979

Kim, K., \& Koo, B. 2002, ApJ, 575, 327

Kim, K., \& Koo, B. 2003, ApJ, 596, 362

Kreysa, E., et al. 1998, Proc. SPIE, 3357, 319

Kuchar, T. A., \& Clark, F. O. 1997, ApJ, 488, 224

Kurtz, S., Churchwell, E., \& Wood, D. O. S. 1994, ApJS, 91, 659

Kurtz, S. E., Watson, A. M., Hofner, P., \& Otte, B. 1999, ApJ, 514, 232

Kurtz, S., Cesaroni, R., Churchwell, E., Hofner, P., \& Walmsley, C. M. 2000, Protostars and Planets IV, 299 (Tucson: Arizona Press) ed. Mannings, Boss, Russell

Lada, C. J., \& Lada, E. A. 2003, ARA\&A, 41, 57

Lebrón, M. E., Rodríguez, L. F., \& Lizano, S. 2001, ApJ, 560, 806

Lekht, E. E. 2000, A\&AS, 141, 185

Little, L. T., Heaton, B. D., \& Dent, W. R. F. 1990, A\&A, 232, 173

McCutcheon, W. H., Sato, T., Purton, C. R., Matthews, H. E., \& Dewdney, P. E. 1995, AJ, 110, 1762

Macdonald, G. H., Gibb, A. G., Habing, R. J., \& Millar, T. J. 1996, A\&AS, 119, 333

Marti, J., Rodriguez, L. F., \& Reipurth, B. 1993, ApJ, 416, 208

Mehringer, D. M., Goss, W. M., Lis, D. C., Palmer, P., \& Menten, K. M. 1998, ApJ, 493, 274

Molinari, S., Brand, J., Cesaroni, R., \& Palla, F. 2000, A\&A, 355, 617

Minchin, N. R., Ward-Thompson, D., \& White, G. J. 1995, A\&A, 298, 894

Moreno-Corral, M. A., Chavarria, K. C., de Lara, E., \& Wagner, S. 1993, A\&A, 273,619

Moriarty-Schieven, G. H., Snell, R. L., \& Hughes, V. A. 1991, ApJ, 374, 169

Motte, F., Schilke, P., \& Lis, D. C. 2003, ApJ, 582, 277

Mueller, K. E., Shirley, Y. L., Evans, N. J., \& Jacobson, H. R. 2002, ApJS, 143, 469

Nielbock, M., Chini, R., Jütte, M., \& Manthey, E. 2001, A\&A, 377, 273

Nyman, L.-Å., et al. 2001, The Messenger, 106, 40

Okamoto, Y. K., Kataza, H., Yamashita, T., et al. 2003, ApJ, 584, 368

Palagi, F., Cesaroni, R., Comoretto, G., Felli, M., \& Natale, V. 1993, A\&AS, 101,153

Palumbo, M. E., Tielens, A. G. G. M., \& Tokunaga, A. T. 1995, ApJ, 449, 674

Persi, P., Tapia, M., Roth, M., Marenzi, A. R., Testi, L., \& Vanzi, L. 2003, A\&A, 397, 227

Pratap, P., Megeath, S. T., \& Bergin, E. A. 1999, ApJ, 517, 799

Privett, G., Jenness, T., \& Matthews, H. 1998, Starlink User Note 213, Starlink Project, CCLRC

Rengarajan, T. N., \& Ho, P. T. P. 1996, ApJ, 465, 363

Richardson, K. J., White, G. J., Avery, L. W., \& Woodsworth, A. W. 1987, A\&A, 174, 197

Richer, J. S., Padman, R., Ward-Thompson, D., Hills, R. E., \& Harris, A. I. 1993 MNRAS, 262,839

Rodriguez, L. F., Garay, G., Curiel, S., et al. 1994, ApJ, 430, L65

Sandell, G., \& Knee, L. B. G. 2001, ApJ, 546, L49

Sargent, A. I. 1977, ApJ, 218, 736

Sargent, A. I. 1979, ApJ, 233, 163

Sault, R. J., Teuben, P. J., \& Wright, M. C. H. 1995, Astronomical Data Analysis Software \& Systems IV, ed. R. A. Shaw, H. E. Payne, \& J. J. E. Hayes, ASP Conf. Ser., 77, 433

Schutte, A. J., van der Walt, D. J., Gaylard, M. J., \& MacLeod, G. C. 1993, MNRAS, 261, 783

Sharpless, S. 1959, ApJS, 4, 257

Shepherd, D. S., \& Churchwell, E. 1996, ApJ, 457, 267

Shirley, Y. L., Evans, N. J., Young, K. E., Knez, C., \& Jaffe, D. T. 2003, ApJS, 149,375

Smith, N., Jones, T. J., Gehrz, R. D., Klebe, D., \& Creech-Eakman, M. J. 2001, AJ, 121, 984

Sridharan, T. K., Beuther, H., Schilke, P., Menten, K. M., \& Wyrowski, F. 2002, ApJ, 566, 931 
Szymczak, M., Hrynek, G., \& Kus, A. J. 2000, A\&AS, 143, 269

Testi, L., Olmi, L., Hunt, L., et al. 1995, A\&A, 303, 881

Testi, L., Hofner, P., Kurtz, S., \& Rupen, M. 2000, A\&A, 359, L5

Thompson, M. A., \& MacDonald, G. H. 1999, A\&AS, 135, 531

Thompson, M. A., Hatchell, J. H., Macdonald, G. H., \& Millar, T. J. 2004, in preparation

Tieftrunk, A. R., Megeath, S. T., Wilson, T. L., \& Rayner, J. T. 1998, A\&A, 336, 991

Torrelles, J. M., Verdes-Montenegro, L., Ho, P. T. P., Rodriguez, L. F., \& Canto, J. 1993, ApJ, 410, 202

Tothill, N. F. H., White, G. J., Matthews, H. E., et al. 2002, ApJ, 580, 285

Walsh, A. J., Burton, M. G., Hyland, A. R., \& Robinson, G. 1998, MNRAS, 301, 640

Walsh, A. J., Macdonald, G. H., Alvey, N. D. S., Burton, M. G., \& Lee, J.-K. 2003, A\&A, 410, 597

Watson, A. M., \& Hanson, M. M. 1997, ApJ, 490, L165

Watson, C., Araya, E., Sewilo, M., Churchwell, E., Hofner, P., \& Kurtz, S. 2003, ApJ, 587, 714

Watt, S., \& Mundy, L. G. 1999, ApJS, 125, 143

White, G. J., Tothill, N. F. H., Matthews, H. E., et al. 1997, A\&A, 323, 529

Williams, S. J., Fuller, G. A., \& Sridharan, T. K. 2004, A\&A, 417, 115
Wilson, T. L., Hanson, M. M., \& Muders, D. 2003, ApJ, 590, 895 Wink, J. E., Altenhoff, W. J., \& Mezger, P. G. 1982, A\&A, 108, 227 Winnberg, A. 1970, A\&A, 9, 259

Wood, D. O. S., Churchwell, E., \& Salter, C. J. 1988a, ApJ, 325, 694

Wood, D. O. S., Handa, T., Fukui, Y., Churchwell, E., Sofue, Y., \& Iwata, T. 1988b, ApJ, 326, 884

Wood, D. O. S., \& Churchwell, E. 1989a, ApJS, 69, 831 (WC89a)

Wood, D. O. S., \& Churchwell, E. 1989b, ApJ, 340, 265

Wynn-Williams, C. G., Werner, M. W., \& Wilson, W. J. 1974, ApJ, 187, 41

Wyrowski, F., Hofner, P., Schilke, P., et al. 1997, A\&A, 320, L17

Wyrowski, F., Schilke, P., Walmsley, C. M., \& Menten, K. M. 1999, ApJ, 514, L43

Val'tts, I. E., Ellingsen, S. P., Slysh, V. I., et al. 2000, MNRAS, 317, 315

van der Tak, F. F. S., van Dishoeck, E. F., Evans, N. J., \& Blake, G. A. 2000, ApJ, 537, 283

Yamashita, T., Sato, S., Nagata, T., et al. 1987, A\&A, 177, 258

Zavagno, A., Deharveng, L., Nadeau, D., \& Caplan, J. 2002, A\&A, 394, 225

Zijlstra, A. A., Pottasch, S. R., Engels, D., et al. 1990, MNRAS, 246, 217

Zoonematkermani, S., Helfand, D. J., Becker, R. H., White, R. L., \& Perley, R. A. 1990, ApJS, 74, 181 
M. A. Thompson et al.: A SCUBA imaging survey of UC HII regions, Online Material $p 1$

\section{Online Material}


M. A. Thompson et al.: A SCUBA imaging survey of UC HII regions, Online Material $p 2$

Table 2. Identifications, coordinates, peak and integrated fluxes of each submillimetre source detected in the survey. Multiple sources located in a single jiggle-map are indicated by labels following the UC HII region field name. These labels are the same as those used in Figs. 1 and $2.450 \mu \mathrm{m}$ peak flux measurements indicated by a dagger $\left(^{\dagger}\right)$ are measured from smoothed images and the flux density unit in this case is Janskys per 14" beam. The resolution of the unsmoothed images is $8^{\prime \prime}$ at $450 \mu \mathrm{m}$ and $14^{\prime \prime}$ at $850 \mu \mathrm{m}$. Upper limits for non-detections at $450 \mu \mathrm{m}$ are indicated. Where it was not possible to measure a $450 \mu \mathrm{m}$ flux, either due to the smaller FOV of the short wavelength array or calibration problems this is indicated by an ellipsis (...). Quoted errors include calibration uncertainties of $30 \%$ at $450 \mu \mathrm{m}$ and $10 \%$ at $850 \mu \mathrm{m}$. All source positions were measured from the $850 \mu \mathrm{m}$ images unless otherwise stated.

\begin{tabular}{|c|c|c|c|c|c|c|c|}
\hline \multirow[t]{2}{*}{$\overline{\text { UCHII field }}$} & \multirow[t]{2}{*}{ Source } & \multirow{2}{*}{$\begin{array}{l}\text { RA (J2000) } \\
(\mathrm{h} \text { min s) }\end{array}$} & \multirow{2}{*}{$\begin{array}{l}\text { Dec (J2000) } \\
\left({ }^{\circ}, \prime^{\prime \prime}\right)\end{array}$} & \multicolumn{2}{|c|}{$\overline{\text { Peak flux (Jy/beam) }}$} & \multicolumn{2}{|c|}{ Integrated flux density (Jy) } \\
\hline & & & & $450 \mu \mathrm{m}$ & $850 \mu \mathrm{m}$ & $450 \mu \mathrm{m}$ & $850 \mu \mathrm{m}$ \\
\hline G1.13-0.11 & G1.125-0.108SMM & $17: 48: 41.7$ & $-28: 01: 48$ & $26.3 \pm 9.0^{\dagger}$ & $2.8 \pm 0.4$ & $174.3 \pm 55.2$ & $13.4 \pm 2.8$ \\
\hline $\mathrm{G} 4.41+0.13$ & $\mathrm{G} 4.417+0.127 \mathrm{SMM}^{\mathrm{a}}$ & $17: 55: 18.0$ & $-25: 04: 41$ & $<8.1^{\dagger}$ & $1.2 \pm 0.1$ & $<8.1$ & $2.0 \pm 0.3$ \\
\hline G5.48-0.24 (a) & G5.477-0.246SMM & 17:59:03.5 & $-24: 20: 55$ & $<9.7$ & $0.7 \pm 0.1$ & $<9.7$ & $4.4 \pm 0.5$ \\
\hline G5.48-0.24 (b) & G5.479-0.254SMM & 17:59:05.5 & $-24: 21: 03$ & $<9.7$ & $0.6 \pm 0.1$ & $<9.7$ & $3.0 \pm 0.4$ \\
\hline G5.89-0.39 & G5.887-0.394SMM & $18: 00: 31.0$ & $-24: 04: 01$ & $55.7 \pm 17.1$ & $19.4 \pm 2.0$ & $250.6 \pm 80.2$ & $46.5 \pm 5.3$ \\
\hline G5.97-1.17 (a) & G5.972-1.159SMM & 18:03:37.1 & $-24: 22: 13$ & $\ldots$ & $1.3 \pm 0.2$ & & $4.9 \pm 0.8$ \\
\hline G5.97-1.17 (b) & G5.974-1.175SMM & 18:03:41.1 & $-24: 22: 37$ & $20.8 \pm 6.7^{\dagger}$ & $3.6 \pm 0.4$ & $69.7 \pm 21.7$ & $16.4 \pm 2.5$ \\
\hline G6.55-0.10 & G6.551-0.099SMM & 18:00:50.3 & $-23: 20: 36$ & $<7.8$ & $1.8 \pm 0.3$ & $<7.8$ & $11.1 \pm 2.1$ \\
\hline G8.14+0.23 (a) & G8.143+0.219SMM ${ }^{b}$ & 18:03:02.9 & $-21: 48: 08$ & $25.8 \pm 7.9^{\dagger}$ & $4.2 \pm 0.4$ & $91.8 \pm 27.8$ & $26.4 \pm 2.9$ \\
\hline $\mathrm{G} 8.14+0.23(\mathrm{~b})$ & G8.140+0.224SMM ${ }^{b}$ & 18:03:01.5 & $-21: 48: 10$ & $23.6 \pm 7.2^{\dagger}$ & & $152.5 \pm 46.0$ & \\
\hline $\mathrm{G} 8.14+0.23(\mathrm{c})$ & G8.135+0.245SMM & $18: 02: 56.0$ & $-21: 47: 47$ & $13.1 \pm 4.2^{\dagger}$ & $1.7 \pm 0.2$ & $73.7 \pm 22.2$ & $7.0 \pm 0.8$ \\
\hline $\mathrm{G} 8.14+0.23(\mathrm{~d})$ & G8.136+0.212SMM & $18: 03: 03.5$ & $-21: 48: 42$ & $\ldots$ & $1.1 \pm 0.1$ & $\ldots$ & $2.4 \pm 0.3$ \\
\hline G8.67-0.36 (a) & $\mathrm{G} 8.683-0.368 \mathrm{SMM}^{\mathrm{e}}$ & $18: 06: 23.4$ & $-21: 37: 09$ & $\ldots$ & $4.1 \pm 0.5$ & $\ldots$ & $11.9 \pm 2.3$ \\
\hline G8.67-0.36 (b) & G8.671- & 19.4 & $-21: 37: 27$ & $54.5 \pm 16.6$ & $12.2 \pm 1.3$ & $291.3 \pm 92.3$ & $32.3 \pm 5.0$ \\
\hline G9.62+0.20 & G9.621 & 15.1 & $-20: 31: 34$ & $38.4 \pm 11.8$ & $9.3 \pm 1.0$ & $168.1 \pm 53.3$ & $21.7 \pm 3.8$ \\
\hline G9.88-0.75 (a) & G9.880 & $0: 19.2$ & $-20: 45: 31$ & $14.4 \pm 4.7^{\dagger}$ & $1.9 \pm 0.2$ & $104.3 \pm 35.3$ & $13.8 \pm 1.9$ \\
\hline G9.88-0.75 (b) & G9.872 & 18.0 & $-20: 45: 57$ & $11.8 \pm 4.0^{\dagger}$ & $1.5 \pm 0.2$ & $91.2 \pm 28.7$ & $8.1 \pm 1.1$ \\
\hline G10.10+0.74 & 38SMM & 13.4 & $-19: 50: 34$ & $<5.1$ & $0.8 \pm 0.1$ & $<5.1$ & $1.0 \pm 0.1$ \\
\hline G10.15-0.34 & 5SMM & 21.8 & $-20: 19: 30$ & $17.0 \pm 6.1^{\dagger}$ & $2.2 \pm 0.4$ & $83.1 \pm 28.0$ & $10.6 \pm 2.4$ \\
\hline-0.15 (a) & MM & 6.2 & $-20: 06: 02$ & $28.5 \pm 8.7$ & $6.3 \pm 0.7$ & $254.0 \pm 78.3$ & \pm 3.5 \\
\hline$-0.15(b)$ & MM & 2.7 & $-20: 06: 09$ & $12.7 \pm 4.1$ & $1.9 \pm 0.3$ & $43.3 \pm 13.3$ & \pm 0.7 \\
\hline G10.62-0.38 & SMM & 29.0 & $-19: 55: 51$ & $120.3 \pm 36.2$ & $27.5 \pm 2.8$ & $730.5 \pm 256.9$ & $65.3 \pm 9.1$ \\
\hline G10.84-2.59 & G10.842-2.594SMM & 18:19:12.7 & $-20: 47: 31$ & $24.2 \pm 7.5$ & $5.6 \pm 0.6$ & $96.6 \pm 30.7$ & $14.8 \pm 3.3$ \\
\hline G11.11-0.34 (a) & G11.110-0.401SMM & $18: 11: 32.4$ & $-19: 30: 44$ & $16.2 \pm 5.1$ & $2.7 \pm 0.3$ & $92.1 \pm 28.2$ & $15.8 \pm 3.3$ \\
\hline G11.11-0.34 (b) & G11.117-0.415SMM & $: 11: 36.2$ & $-19: 30: 48$ & & $0.9 \pm 0.2$ & & $4.9 \pm 0.7$ \\
\hline G11.94-0.62 (a) & G11.936-0.618SMM & 18:14:01.4 & $-18: 53: 29$ & $29.9 \pm$ & $5.8 \pm 0.6$ & $337.0 \pm$ & $20.1 \pm 2.8$ \\
\hline G11.94-0.62 (b) & G11.918-0.615SMM & $18: 13: 58.7$ & $-18: 54: 23$ & & $3.9 \pm 0.4$ & & $10.9 \pm 1.6$ \\
\hline G12.43-0.05 (a) & G12.429-0.050SMM & $18: 12: 55.0$ & $-18: 11: 14$ & $<3.9^{\dagger}$ & $0.4 \pm 0.1$ & $<3.9$ & $2.4 \pm 0.3$ \\
\hline G12.43-0.05 (b) & G12.439-0.060SMM & $18: 12: 58.5$ & $-18: 10: 58$ & $<3.9^{\dagger}$ & $0.4 \pm 0.1$ & $<3.9$ & $2.5 \pm 0.3$ \\
\hline G12.43-0.05 (c) & G12.437-0.055SMM & $18: 12: 57.0$ & $-18: 10: 58$ & $<3.9^{\dagger}$ & $0.4 \pm 0.1$ & $<3.9$ & $3.9 \pm 0.4$ \\
\hline G12.90-0.25 (a) & G12.908-0.261SMM & $18: 14: 39.6$ & $-17: 52: 04$ & $53.5 \pm 16.1^{\dagger}$ & $7.0 \pm 0.8$ & $254.3 \pm 80.3$ & $22.1 \pm 3.5$ \\
\hline G12.90-0.25 (b) & G12.900-0.243SMM & $18: 14: 34.6$ & $-17: 51: 56$ & $13.3 \pm 4.3^{\dagger}$ & $1.5 \pm 0.3$ & $53.3 \pm 17.2$ & $3.8 \pm 0.7$ \\
\hline G13.19+0.04 (a) & G13.177+0.059SMM & 18:14:01.2 & $-17: 28: 43$ & & $3.7 \pm 0.5$ & & $11.0 \pm 2.3$ \\
\hline $\mathrm{G} 13.19+0.04(\mathrm{~b})$ & G13.186+0.028SMM & 18:14:09.2 & $-17: 29: 06$ & & $0.7 \pm 0.3$ & & $1.8 \pm 0.3$ \\
\hline $\mathrm{G} 13.87+0.28$ & G13.873+0.279SMM & $18: 14: 36.3$ & $-16: 45: 44$ & $16.3 \pm 5.2$ & $3.8 \pm 0.5$ & $100.1 \pm 33.5$ & $16.7 \pm 4.9$ \\
\hline G15.04-0.68 (a) & G15.026-0.674SMM ${ }^{\mathrm{b}}$ & $18: 20: 23.1$ & $-16: 11: 58$ & $33.1 \pm 10.4$ & $12.0 \pm 1.5$ & $416.9 \pm 127.0$ & $65.2 \pm 9.0$ \\
\hline G15.04-0.68 (b) & G15.031-0.676SMM ${ }^{\mathrm{b}}$ & $18: 20: 24.1$ & $-16: 11: 43$ & $33.9 \pm 10.6$ & $\ldots$ & $139.4 \pm 43.9$ & $\cdots$ \\
\hline G15.04-0.68 (c) & G15.031-0.669SMM ${ }^{\mathrm{b}}$ & $18: 20: 22.7$ & $-16: 11: 32$ & $45.6 \pm 14.0$ & & $343.5 \pm 105.3$ & \\
\hline G18.15-0.28 & G18.149-0.286SMM & $18: 25: 01.8$ & $-13: 15: 35$ & $17.3 \pm 5.4^{\dagger}$ & $2.0 \pm 0.3$ & $118.7 \pm 40.7$ & $4.7 \pm 1.1$ \\
\hline G18.30-0.39 (a) & G18.302-0.286SMM ${ }^{b}$ & $18: 25: 41.8$ & $-13: 10: 19$ & $17.3 \pm 5.4^{\dagger}$ & $2.4 \pm 0.4$ & $111.2 \pm 34.0$ & $16.7 \pm 0.3$ \\
\hline G18.30-0.39 (b) & G18.298-0.392SMM ${ }^{\mathrm{b}}$ & $18: 25: 42.2$ & $-13: 10: 37$ & $20.5 \pm 6.4^{\dagger}$ & & $143.9 \pm 1.1$ & \\
\hline G19.07-0.27 & G19.077-0.289SMM & $18: 26: 49.0$ & $-12: 26: 25$ & $21.7 \pm 6.6$ & $4.1 \pm 0.4$ & $110.6 \pm 35.9$ & $13.1 \pm 2.8$ \\
\hline $\mathrm{G} 19.49+0.14$ (a) & G19.487+0.136SMM & $18: 26: 03.7$ & $-11: 52: 45$ & $4.6 \pm 1.8^{\dagger}$ & $0.7 \pm 0.2$ & $13.3 \pm 5.0$ & $1.8 \pm 0.6$ \\
\hline $\mathrm{G} 19.49+0.14$ (b) & G19.474+0.158SMM & $18: 25: 57.6$ & $-11: 52: 49$ & $4.0 \pm 1.7^{\dagger}$ & $0.9 \pm 0.2$ & $7.6 \pm 2.4$ & $2.1 \pm 0.5$ \\
\hline $\mathrm{G} 19.49+0.14(\mathrm{c})$ & G19.486+0.148SMM & 18:26:01.1 & $-11: 52: 29$ & $9.4 \pm 3.1^{\dagger}$ & $1.0 \pm 0.2$ & $24.2 \pm 9.1$ & $2.8 \pm 0.7$ \\
\hline G19.61-0.23 & G19.609-0.235SMM & $18: 27: 38.2$ & $-11: 56: 38$ & $84.6 \pm 25.4$ & $19.8 \pm 2.0$ & $191.7 \pm 59.4$ & $26.2 \pm 3.0$ \\
\hline G20.08-0.14 (a) & G20.081-0.136SMM & $18: 28: 10.5$ & $-11: 28: 50$ & $46.3 \pm 13.9$ & $8.2 \pm 0.8$ & $77.5 \pm 23.9$ & $13.8 \pm 1.6$ \\
\hline
\end{tabular}


M. A. Thompson et al.: A SCUBA imaging survey of UC HII regions, Online Material p 3

Table 2. continued.

\begin{tabular}{|c|c|c|c|c|c|c|c|}
\hline \multirow[t]{2}{*}{ UCHII field } & \multirow[t]{2}{*}{ Source } & \multirow{2}{*}{$\begin{array}{l}\text { RA (J2000) } \\
(\mathrm{h} \text { min s) }\end{array}$} & \multirow{2}{*}{$\begin{array}{l}\text { Dec (J2000) } \\
\left({ }^{\circ}, \prime^{\prime \prime}\right)\end{array}$} & \multicolumn{2}{|c|}{$\overline{\text { Peak flux (Jy/beam) }}$} & \multicolumn{2}{|c|}{ Integrated flux density (Jy) } \\
\hline & & & & $450 \mu \mathrm{m}$ & $850 \mu \mathrm{m}$ & $450 \mu \mathrm{m}$ & $850 \mu \mathrm{m}$ \\
\hline G20.08-0.14 (b) & G20.073-0.143SMM & $18: 28: 11.0$ & $-11: 29: 26$ & $<3.0$ & $0.4 \pm 0.1$ & $<3.0$ & $0.8 \pm 0.1$ \\
\hline G20.99+0.09 (a) & G20.990+0.089SMM & $18: 29: 05.0$ & $-10: 34: 13$ & $<2.7^{\dagger}$ & $0.6 \pm 0.1$ & $<2.7$ & $3.7 \pm 0.4$ \\
\hline G20.99+0.09 (b) & G20.981+0.094SMM & $18: 29: 02.8$ & $-10: 34: 36$ & $7.3 \pm 2.4^{\dagger}$ & $1.2 \pm 0.1$ & $25.4 \pm 8.7$ & $7.2 \pm 1.0$ \\
\hline G22.76-0.49 & G22.756-0.485SMM & $18: 34: 28.0$ & $-09: 16: 12$ & $<2.4$ & $0.4 \pm 0.1$ & $<2.4$ & $1.6 \pm 0.3$ \\
\hline G23.46-0.20 & G23.454-0.203SMM & $18: 34: 45.1$ & $-08: 31: 15$ & $10.8 \pm 3.4^{\dagger}$ & $1.2 \pm 0.2$ & $14.0 \pm 4.3$ & $1.3 \pm 0.2$ \\
\hline $\mathrm{G} 23.71+0.17$ & G23.708+0.169SMM & $18: 33: 53.5$ & $-08: 07: 25$ & $14.7 \pm 4.5$ & $2.9 \pm 0.3$ & $56.2 \pm 17.3$ & $15.1 \pm 2.9$ \\
\hline G23.87-0.12 (a) & G23.866-0.114SMM & $18: 35: 12.1$ & $-08: 06: 49$ & $10.6 \pm 3.6^{\dagger}$ & $0.9 \pm 0.1$ & $42 \pm 12.7$ & $6.0 \pm 1.0$ \\
\hline G23.87-0.12 (b) & $\mathrm{G} 23.872-0.122 \mathrm{SMM}^{\mathrm{c}}$ & $18: 35: 14.6$ & $-08: 06: 43$ & $12.1 \pm 4.0^{\dagger}$ & $1.1 \pm 0.2$ & $78.7 \pm 23.9$ & $5.2 \pm 0.7$ \\
\hline G23.87-0.12 (c) & G23.866-0.124SMMc & $18: 35: 14.3$ & $-08: 07: 07$ & & $1.4 \pm 0.2$ & .. & $4.7 \pm 0.7$ \\
\hline G23.87-0.12 (d) & G23.861-0.125SMM & $18: 35: 14.0$ & $-08: 07: 24$ & $11.3 \pm 3.7^{\dagger}$ & $1.3 \pm 0.2$ & $28.9 \pm 9.2$ & $2.8 \pm 0.4$ \\
\hline G23.96+0.15 (a) & G23.944+0.159SMM & $18: 34: 21.9$ & $-07: 55: 08$ & $12.1 \pm 4.0^{\dagger}$ & $1.2 \pm 0.2$ & $38.7 \pm 11.7$ & $2.5 \pm 0.4$ \\
\hline G23.96+0.15 (b) & G23.955+0.148SMM & $18: 34: 25.5$ & $-07: 54: 52$ & $22.1 \pm 6.8^{\dagger}$ & $2.0 \pm 0.2$ & $52.5 \pm 16.2$ & $6.9 \pm 0.8$ \\
\hline G23.96+0.15 (c) & G23.952+0.152SMM & $18: 34: 24.3$ & $-07: 54: 55$ & $23.5 \pm 7.2^{\dagger}$ & $2.3 \pm 0.3$ & $87.1 \pm 26.5$ & $7.7 \pm 1.0$ \\
\hline G23.96+0.15 (d) & G23.949+0.163SMM ${ }^{\mathrm{a}}$ & $18: 34: 21.7$ & $-07: 54: 47$ & $11.3 \pm 3.7^{\dagger}$ & $1.2 \pm 0.2$ & $58.3 \pm 17.5$ & $2.2 \pm 0.3$ \\
\hline G24.47+0.49 (a) & $\mathrm{G} 24.469+0.495 \mathrm{SMM}^{\mathrm{a}}$ & $18: 34: 08.4$ & $-07: 17: 52$ & $14.9 \pm 4.7^{\dagger}$ & $1.2 \pm 0.2$ & $115.1 \pm 36.1$ & $5.7 \pm 0.7$ \\
\hline G24.47+0.49 (b) & G24.474+0.487SMM & $18: 34: 10.7$ & $-07: 17: 52$ & $14.5 \pm 4.6^{\dagger}$ & $1.7 \pm 0.2$ & $65.0 \pm 20.2$ & $12.4 \pm 1.6$ \\
\hline $\mathrm{G} 24.47+0.49(\mathrm{c})$ & 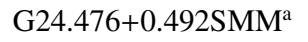 & 18:34:09.7 & $-07: 17: 37$ & $18.2 \pm 5.7^{\dagger}$ & $1.4 \pm 0.2$ & $112.0 \pm 36.2$ & $3.7 \pm 0.4$ \\
\hline G24.68-0.16 (a) & G24.674-0.152SMM & $18: 36: 50.3$ & $-07: 24: 50$ & $46.2 \pm 14.0^{\dagger}$ & $4.0 \pm 0.4$ & $275.8 \pm 89.6$ & $17.4 \pm 2.4$ \\
\hline G24.68-0.16 (b) & G24.668-0.161SMM & $18: 36: 51.6$ & $-07: 25: 24$ & $14.0 \pm 4.6^{\dagger}$ & $1.1 \pm 0.2$ & $86.5 \pm 27.7$ & $9.0 \pm 1.1$ \\
\hline G24.68-0.16 (c) & G24.683-0.150SMM & $18: 36: 50.9$ & $-07: 24: 18$ & $13.1 \pm 4.3^{\dagger}$ & $0.9 \pm 0.2$ & $20.4 \pm 6.3$ & $3.0 \pm 0.4$ \\
\hline G25.38-0.18 & G25.382-0.184SMM & $18: 38: 15.7$ & $-06: 47: 58$ & & $2.7 \pm 0.3$ & & $12.4 \pm 1.7$ \\
\hline $\mathrm{G} 25.65+1.05$ & G25.650+1.049SMM & $18: 34: 21.1$ & $-05: 59: 43$ & $45.8 \pm 13.8$ & $8.6 \pm 0.9$ & $258.8 \pm 84.5$ & $28.7 \pm 3.9$ \\
\hline G25.72+0.05 (a) & G25.710+0.043SMM & $18: 38: 03.4$ & $-06: 24: 16$ & $21.9 \pm 6.8^{\dagger}$ & $2.2 \pm 0.3$ & $85.4 \pm 29.0$ & $7.4 \pm 1.8$ \\
\hline G25.72+0.05 (b) & G25.714+0.046SMM & 18:38:03.2 & $-06: 23: 56$ & $12.7 \pm 4.1^{\dagger}$ & $1.0 \pm 0.2$ & $64.7 \pm 21.4$ & $2.9 \pm 0.4$ \\
\hline G26.54+0.42 (a) & G26.529+0.422SMM & $18: 38: 12.8$ & $-05: 30: 10$ & $<4.8^{\dagger}$ & $0.5 \pm 0.2$ & $<4.8$ & $1.6 \pm 0.2$ \\
\hline G26.54+0.42 (b) & 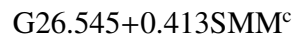 & $18: 38: 16.5$ & $-05: 29: 34$ & $8.5 \pm 3.0^{\dagger}$ & $1.0 \pm 0.2$ & $41.9 \pm 12.7$ & $6.5 \pm 1.5$ \\
\hline G26.54+0.42 (c) & 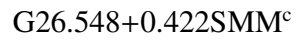 & $18: 38: 15.0$ & $-05: 29: 08$ & & $0.7 \pm 0.2$ & & $4.0 \pm 0.7$ \\
\hline G27.28+0.15 (a) & G27.267+0.146SMM & $18: 40: 33.4$ & $-04: 58: 22$ & $7.8 \pm 2.8^{\dagger}$ & $0.6 \pm 0.2$ & $42.6 \pm 13.4$ & $5.8 \pm 0.8$ \\
\hline $\mathrm{G} 27.28+0.15(\mathrm{~b})$ & G27.280+0.144SMM & $18: 40: 35.2$ & $-04: 57: 46$ & $13.1 \pm 4.2^{\dagger}$ & $1.7 \pm 0.2$ & $33.4 \pm 11.1$ & $5.9 \pm 0.8$ \\
\hline $\mathrm{G} 27.28+0.15$ (c) & G27.285+0.149SMM & $18: 40: 34.9$ & $-04: 57: 20$ & $13.8 \pm 4.4^{\dagger}$ & $1.7 \pm 0.2$ & $45.5 \pm 15.2$ & $8.2 \pm 1.2$ \\
\hline $\mathrm{G} 27.28+0.15$ (d) & G27.296+0.151SMM & $18: 40: 35.5$ & $-04: 56: 41$ & $8.2 \pm 2.9^{\dagger}$ & $1.0 \pm 0.2$ & $23.2 \pm 7.4$ & $3.9 \pm 0.7$ \\
\hline G27.49+0.19 (a) & G27.494+0.187SMM & $18: 40: 49.7$ & $-04: 45: 10$ & $11.4 \pm 3.8^{\dagger}$ & $1.2 \pm 0.2$ & $24.5 \pm 8.4$ & $4.6 \pm 1.1$ \\
\hline $\mathrm{G} 27.49+0.19(\mathrm{~b})$ & G27.504+0.193SMM & $18: 40: 49.4$ & $-04: 44: 28$ & $<3.6^{\dagger}$ & $0.6 \pm 0.2$ & $<3.6$ & $2.5 \pm 0.5$ \\
\hline G28.20-0.05 & G28.200-0.051SMM & $18: 42: 58.4$ & $-04: 14: 01$ & $61.6 \pm 18.5$ & $9.4 \pm 0.9$ & $244.9 \pm 77.8$ & $19.6 \pm 2.6$ \\
\hline G28.29-0.36 (a) & G28.305-0.388SMMa & $18: 44: 22.2$ & $-04: 17: 39$ & $13.6 \pm 4.3^{\dagger}$ & $1.1 \pm 0.1$ & $44.6 \pm 14.1$ & $4.2 \pm 0.6$ \\
\hline G28.29-0.36 (b) & G28.301-0.384SMM ${ }^{\mathrm{a}}$ & $18: 44: 20.7$ & $-04: 17: 44$ & $14.4 \pm 4.5^{\dagger}$ & $1.4 \pm 0.2$ & $48.9 \pm 15.9$ & $4.1 \pm 0.6$ \\
\hline G28.29-0.36 (c) & G28.288-0.365SMM ${ }^{\mathrm{c}}$ & $18: 44: 15.3$ & $-04: 17: 55$ & $19.5 \pm 6.0^{\dagger}$ & $2.2 \pm 0.2$ & $126.3 \pm 41.7$ & $11.8 \pm 1.7$ \\
\hline G28.29-0.36 (d) & G28.283-0.363SMM ${ }^{\mathrm{c}}$ & $18: 44: 14.3$ & $-04: 18: 07$ & & $1.8 \pm 0.2$ & & $5.4 \pm 0.7$ \\
\hline G28.60-0.36 (a) & G28.596-0.362SMM & $18: 44: 48.4$ & $-04: 01: 24$ & $<5.1$ & $0.6 \pm 0.1$ & $<5.1$ & $5.2 \pm 0.9$ \\
\hline G28.60-0.36 (b) & G28.603-0.374SMM ${ }^{\mathrm{a}}$ & $18: 44: 51.8$ & $-04: 01: 21$ & $<5.1$ & $0.7 \pm 0.1$ & $<5.1$ & $3.5 \pm 0.4$ \\
\hline G28.60-0.36 (c) & G28.602-0.380SMM & $18: 44: 53.0$ & $-04: 01: 36$ & $<5.1$ & $0.5 \pm 0.1$ & $<5.1$ & $2.2 \pm 0.3$ \\
\hline G28.80+0.17 (a) & G28.806+0.180SMM & $18: 43: 15.7$ & $-03: 35: 22$ & & $1.1 \pm 0.1$ & & $7.6 \pm 1.1$ \\
\hline G28.80+0.17 (b) & G28.812+0.168SMM & $18: 43: 18.8$ & $-03: 35: 19$ & & $1.6 \pm 0.2$ & & $6.3 \pm 0.8$ \\
\hline G28.83-0.23 & G28.834-0.209SMM & $18: 44: 42.0$ & $-03: 44: 30$ & $<5.1$ & $1.0 \pm 0.2$ & $<5.1$ & $2.0 \pm 0.5$ \\
\hline G29.96-0.02 (a) & G29.956-0.017SMM & $18: 46: 04.0$ & $-02: 39: 22$ & $60.0 \pm 18.0$ & $11.5 \pm 1.2$ & $200.4 \pm 62.1$ & $19.2 \pm 2.3$ \\
\hline G29.96-0.02 (b) & G29.938-0.012SMM & 18:46:01.0 & $-02: 40: 12$ & $3.6 \pm 1.3$ & $0.6 \pm 0.2$ & $9.6 \pm 3.1$ & $1.4 \pm 0.3$ \\
\hline G29.96-0.02 (c) & G29.972-0.011SMM & 18:46:04.4 & $-02: 38: 20$ & $4.8 \pm 1.6$ & $0.7 \pm 0.2$ & $15.0 \pm 4.9$ & $1.9 \pm 0.5$ \\
\hline
\end{tabular}


Table 2. continued.

\begin{tabular}{|c|c|c|c|c|c|c|c|}
\hline \multirow[t]{2}{*}{$\overline{\text { UCHII field }}$} & \multirow[t]{2}{*}{ Source } & \multirow{2}{*}{$\begin{array}{l}\text { RA (J2000) } \\
(\mathrm{h} \text { min s) }\end{array}$} & \multirow{2}{*}{$\begin{array}{l}\begin{array}{l}\text { Dec (J2000) } \\
\left({ }^{\circ}, \prime^{\prime \prime}\right)\end{array} \\
\end{array}$} & \multicolumn{2}{|c|}{ Peak flux (Jy/beam) } & \multicolumn{2}{|c|}{ Integrated flux density (Jy) } \\
\hline & & & & $450 \mu \mathrm{m}$ & $850 \mu \mathrm{m}$ & $450 \mu \mathrm{m}$ & $850 \mu \mathrm{m}$ \\
\hline G29.96-0.02(d) & G29.964-0.011SMM & $18: 46: 03.7$ & $-02: 38: 48$ & $8.3 \pm 2.6$ & $1.1 \pm 0.2$ & $20.4 \pm 7.0$ & $2.1 \pm 0.6$ \\
\hline $\mathrm{G} 30.54+0.02$ & G30.535+0.020SMM & $18: 46: 59.5$ & $-02: 07: 26$ & $18.9 \pm 5.7^{\dagger}$ & $2.0 \pm 0.3$ & $75.0 \pm 24.9$ & $5.3 \pm 1.2$ \\
\hline G30.78-0.02 (a) & G30.785-0.022SMM & $18: 47: 35.9$ & $-01: 55: 13$ & $31.5 \pm 9.9^{\dagger}$ & $2.9 \pm 0.4$ & $130.8 \pm 45.1$ & $9.6 \pm 1.7$ \\
\hline G30.78-0.02 (b) & G30.786-0.028SMM ${ }^{\mathrm{a}}$ & $18: 47: 37.4$ & $-01: 55: 22$ & $19.4 \pm 6.5^{\dagger}$ & $2.1 \pm 0.3$ & $106.6 \pm 35.7$ & $7.1 \pm 1.4$ \\
\hline $\mathrm{G} 31.28+0.06$ & G31.280+0.061SMM & $18: 48: 12.5$ & $-01: 26: 31$ & $53.9 \pm 16.4$ & $6.6 \pm 0.7$ & $224.5 \pm 73.1$ & $24.9 \pm 6.6$ \\
\hline G31.40-0.26 (a) & G31.396-0.258SMM & $18: 49: 33.2$ & $-01: 29: 05$ & $52.6 \pm 15.9$ & $6.5 \pm 0.7$ & $160.4 \pm 51.1$ & $10.4 \pm 1.4$ \\
\hline G31.40-0.26 (b) & G31.387-0.269SMM & $18: 49: 34.7$ & $-01: 29: 52$ & $<5.1$ & $0.7 \pm 0.1$ & $<5.1$ & $1.9 \pm 0.5$ \\
\hline $\mathrm{G} 32.80+0.19$ & G32.797+0.190SMM & $18: 50: 30.8$ & $+00: 02: 01$ & $77.2 \pm 23.3$ & $12.0 \pm 1.2$ & $314.0 \pm 99.2$ & $23.8 \pm 3.1$ \\
\hline G33.13-0.09 & G33.132-0.094SMM & 18:52:08.2 & $+00: 08: 09$ & $37.0 \pm 11.3$ & $4.8 \pm 0.5$ & $117.9 \pm 37.6$ & $13.7 \pm 2.1$ \\
\hline G33.92+0.11 & G33.914+0.109SMM & $18: 52: 50.4$ & $+00: 55: 27$ & $42.6 \pm 12.9$ & $6.1 \pm 0.6$ & $226.0 \pm 68.7$ & $21.0 \pm 2.5$ \\
\hline G34.26+0.15 (a) & G34.257+0.152SMM & $18: 53: 18.8$ & $+01: 14: 56$ & $226.9 \pm 68.1$ & $55.7 \pm 5.6$ & $1464.2 \pm 454.3$ & $189.4 \pm 19.1$ \\
\hline G34.26+0.15 (b) & G34.244+0.133SMM & $18: 53: 21.5$ & $+01: 13: 42$ & $\ldots$ & $2.7 \pm 0.3$ & $\ldots$ & $1.5 \pm 0.2$ \\
\hline G34.26+0.15 (c) & G34.257+0.164SMM & $18: 53: 16.2$ & $+01: 15: 14$ & $33.6 \pm 10.3$ & $7.4 \pm 0.7$ & $255.3 \pm 78.8$ & $24.4 \pm 2.5$ \\
\hline $\mathrm{G} 35.02+0.35$ & G35.024+0.349SMM & $18: 54: 00.6$ & $+02: 01: 15$ & $49.9 \pm 15.1$ & $6.3 \pm 0.6$ & $128.3 \pm 41.0$ & $10.0 \pm 1.2$ \\
\hline G35.05-0.52 (a) & G35.039-0.505SMM & $18: 57: 04.9$ & $+01: 38: 43$ & $\ldots$ & $0.5 \pm 0.1$ & $\cdots$ & $1.2 \pm 0.1$ \\
\hline G35.05-0.52 (b) & G35.053-0.518SMM & $18: 57: 09.3$ & $+01: 39: 04$ & $\ldots$ & $1.1 \pm 0.2$ & & $3.0 \pm 0.5$ \\
\hline G35.05-0.52 (c) & G35.049-0.513SMM & $18: 57: 07.6$ & $+01: 39: 00$ & $\ldots$ & $0.6 \pm 0.1$ & $\ldots$ & $2.8 \pm 0.4$ \\
\hline G35.57+0.07 & G35.576+0.067SMM & $18: 56: 01.4$ & $+02: 23: 00$ & $\ldots$ & $1.6 \pm 0.2$ & $\ldots$ & $10.2 \pm 1.5$ \\
\hline G35.58-0.03 & G35.578-0.031SMM & $18: 56: 22.7$ & $+02: 20: 26$ & $\ldots$ & $4.9 \pm 0.5$ & $\ldots$ & $9.9 \pm 1.6$ \\
\hline G37.55-0.11 & G37.545-0.113SMM & 19:00:16.3 & $+04: 03: 11$ & $\ldots$ & $2.2 \pm 0.3$ & 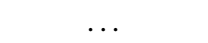 & $7.4 \pm 1.8$ \\
\hline G37.77-0.20 & G37.764-0.216SMM & 19:01:02.3 & $+04: 12: 03$ & $\ldots$ & $2.0 \pm 0.2$ & $\ldots$ & $13.7 \pm 1.8$ \\
\hline G37.87-0.40 & G37.873-0.400SMM & 19:01:53.7 & $+04: 12: 47$ & $\ldots$ & $4.7 \pm 0.5$ & $\ldots$ & $15.1 \pm 2.6$ \\
\hline G39.25-0.06 & G39.254-0.060SMM & 19:03:13.2 & $+05: 35: 47$ & $\ldots$ & $1.4 \pm 0.2$ & $\ldots$ & $6.1 \pm 0.9$ \\
\hline G41.74+0.10 & G41.743+0.097SMM & 19:07:15.7 & $+07: 52: 47$ & $\ldots$ & $0.9 \pm 0.2$ & $\ldots$ & $2.2 \pm 0.6$ \\
\hline G42.42-0.27 (a) & G42.421-0.261SMM & 19:09:48.6 & $+08: 19: 00$ & $6.2 \pm 2.4^{\dagger}$ & $0.6 \pm 0.1$ & $73.3 \pm 23.7$ & $4.3 \pm 1.3$ \\
\hline G42.42-0.27 (b) & G42.434-0.260SMM & $19: 09: 49.8$ & $+08: 19: 44$ & $19.6 \pm 6.1^{\dagger}$ & $1.8 \pm 0.2$ & $26.4 \pm 8.8$ & $2.6 \pm 0.5$ \\
\hline G43.18-0.52 (a) & G43.179-0.519SMM & $19: 12: 09.0$ & $+08: 52: 11$ & $22.3 \pm 6.9$ & $3.9 \pm 0.4$ & $124.1 \pm 38.9$ & $13.2 \pm 2.3$ \\
\hline G43.18-0.52 (b) & G43.158-0.518SMM & $19: 12: 06.4$ & $+08: 51: 05$ & $<5.1$ & $0.6 \pm 0.1$ & $<5.1$ & $1.2 \pm 0.2$ \\
\hline G43.24-0.05 & G43.237-0.047SMM & $19: 10: 33.9$ & $+09: 08: 21$ & $27.1 \pm 8.3$ & $4.7 \pm 0.5$ & $104.7 \pm 32.6$ & $13.3 \pm 1.7$ \\
\hline G43.80-0.13 & G43.795-0.127SMM & $19: 11: 53.9$ & $+09: 35: 50$ & $62.5 \pm 18.8$ & $7.1 \pm 0.7$ & $133.7 \pm 42.2$ & $13.8 \pm 1.7$ \\
\hline $\mathrm{G} 45.07+0.13$ & G45.071+0.131SMM & $19: 13: 22.3$ & $+10: 50: 51$ & $20.3 \pm 6.3$ & $6.7 \pm 0.7$ & $80.2 \pm 27.7$ & $15.5 \pm 1.8$ \\
\hline $\mathrm{G} 45.12+0.13$ & G45.122+0.131SMM & $19: 13: 28.1$ & $+10: 53: 34$ & $12.0 \pm 3.9^{\dagger}$ & $5.0 \pm 0.5$ & $103.5 \pm 37.3$ & $24.6 \pm 2.7$ \\
\hline G45.47+0.05 (a) & G45.467+0.045SMM & $19: 14: 25.8$ & $+11: 09: 28$ & $\ldots$ & $4.5 \pm 0.5$ & $\ldots$ & $16.0 \pm 1.8$ \\
\hline $\mathrm{G} 45.47+0.05(\mathrm{~b})$ & G45.455+0.060SMM & $19: 14: 21.3$ & $+11: 09: 15$ & & $2.8 \pm 0.3$ & & $8.9 \pm 1.1$ \\
\hline G69.54-0.98 & G69.541-0.975SMM & 20:10:09.1 & $+31: 31: 37$ & $62.8 \pm 18.9$ & $8.9 \pm 0.9$ & $470.2 \pm 150.2$ & $32.9 \pm 4.7$ \\
\hline G70.29+1.60 & G70.293+1.600SMM & $20: 01: 45.6$ & $+33: 32: 44$ & $53.6 \pm 16.1$ & $13.6 \pm 1.4$ & $256.4 \pm 79.3$ & $37.6 \pm 4.3$ \\
\hline $\mathrm{G} 76.18+0.13$ (a) & $\mathrm{G} 6.184+0.082 \mathrm{SMM}^{\mathrm{a}}$ & $20: 23: 58.3$ & $+37: 37: 29$ & $8.1 \pm 2.9^{\dagger}$ & $1.1 \pm 0.2$ & $83.9 \pm 30.7$ & $2.7 \pm 0.9$ \\
\hline $\mathrm{G} 76.18+0.13(\mathrm{~b})$ & $\mathrm{G} 6.188+0.097 \mathrm{SMM}^{\mathrm{c}}$ & $20: 23: 55.2$ & $+37: 38: 10$ & $11.8 \pm 3.8^{\dagger}$ & $1.1 \pm 0.2$ & $128.5 \pm 44.7$ & $6.7 \pm 1.9$ \\
\hline $\mathrm{G} 76.18+0.13(\mathrm{c})$ & $\mathrm{G} 6.192+0.090 \mathrm{SMM}^{\mathrm{a}}$ & $20: 23: 57.7$ & $+37: 38: 09$ & & $0.8 \pm 0.2$ & $\ldots$ & $2.7 \pm 0.6$ \\
\hline G76.38-0.62 (a) & G76.385-0.623SMM & $20: 27: 27.5$ & $+37: 22: 49$ & $24.5 \pm 7.6^{\dagger}$ & $3.4 \pm 0.4$ & $192.3 \pm 62.9$ & $17.0 \pm 1.9$ \\
\hline G76.38-0.62 (b) & G76.382-0.620SMM & $20: 27: 26.1$ & $+37: 22: 46$ & $26.8 \pm 8.3^{\dagger}$ & $3.6 \pm 0.4$ & $48.2 \pm 15.0$ & $6.1 \pm 0.7$ \\
\hline G76.38-0.62 (c) & G76.386-0.637SMM $^{\mathrm{d}}$ & $20: 27: 31.1$ & $+37: 22: 25$ & $12.4 \pm 4.2^{\dagger}$ & & $53.1 \pm 18.7$ & \\
\hline G77.97-0.01 (a) & G77.953+0.005SMM & $20: 29: 31.9$ & $+39: 01: 10$ & $8.8 \pm 3.0^{\dagger}$ & $0.8 \pm 0.1$ & $18.5 \pm 6.9$ & $2.6 \pm 0.5$ \\
\hline G77.97-0.01 (b) & G77.962-0.008SMM & $20: 29: 36.8$ & $+39: 01: 09$ & $5.5 \pm 2.2^{\dagger}$ & $0.7 \pm 0.1$ & $9.3 \pm 3.4$ & $1.7 \pm 0.4$ \\
\hline G78.44+2.66 (a) & G78.438+2.660SMM & $20: 19: 39.1$ & $+40: 56: 39$ & $25.2 \pm 7.7^{\dagger}$ & $1.7 \pm 0.2$ & $181.3 \pm 59.8$ & $9.7 \pm 1.2$ \\
\hline $\mathrm{G} 78.44+2.66(\mathrm{~b})$ & G78.446+2.659SMM & $20: 19: 40.7$ & $+40: 57: 02$ & $21.2 \pm 6.5^{\dagger}$ & $1.4 \pm 0.2$ & $119.5 \pm 37.1$ & $7.0 \pm 0.9$ \\
\hline G79.30+0.28 (a) & G79.296+0.281SMM & $20: 32: 29.2$ & $+40: 16: 02$ & $12.2 \pm 4.0^{\dagger}$ & $1.1 \pm 0.1$ & $89.3 \pm 31.0$ & $6.2 \pm 0.9$ \\
\hline $\mathrm{G} 79.30+0.28(\mathrm{~b})$ & $\mathrm{G} 9.316+0.279 \mathrm{SMM}^{\mathrm{e}}$ & $20: 32: 33.8$ & $+40: 16: 55$ & $10.7 \pm 3.5^{\dagger}$ & $1.5 \pm 0.2$ & $35.6 \pm 11.2$ & $3.4 \pm 0.5$ \\
\hline $\mathrm{G} 79.30+0.28(\mathrm{c})$ & G79.306+0.278SMM & $20: 32: 31.8$ & $+40: 16: 23$ & $7.9 \pm 2.8^{\dagger}$ & $0.7 \pm 0.1$ & $51.0 \pm 15.6$ & $3.8 \pm 0.5$ \\
\hline $\mathrm{G} 79.32+1.31$ (a) & G79.308+1.307SMM & $20: 28: 09.7$ & $+40: 52: 50$ & $16.6 \pm 5.2^{\dagger}$ & $1.4 \pm 0.2$ & $59.2 \pm 22.0$ & $4.9 \pm 0.8$ \\
\hline $\mathrm{G} 79.32+1.31(\mathrm{~b})$ & G79.319+1.312SMM & $20: 28: 10.3$ & $+40: 53: 32$ & & $0.8 \pm 0.1$ & & $1.1 \pm 0.2$ \\
\hline $\mathrm{G} 79.32+1.31(\mathrm{c})$ & G79.324+1.291SMM & $20: 28: 16.5$ & $+40: 53: 01$ & $7.4 \pm 2.7^{\dagger}$ & $0.7 \pm 0.1$ & $19.9 \pm 6.9$ & $2.3 \pm 0.3$ \\
\hline $\mathrm{G} 80.87+0.42$ & G80.865+0.419SMM & $20: 36: 52.5$ & $+41: 36: 22$ & $16.6 \pm 5.2^{\dagger}$ & $3.4 \pm 0.4$ & $193.2 \pm 63.5$ & $13.6 \pm 1.9$ \\
\hline G106.80+5.31 (a) & G106.794+5.313SMM & $22: 19: 16.7$ & $+63: 18: 42$ & $30.0 \pm 9.5$ & $6.8 \pm 0.7$ & $433.1 \pm 156.7$ & $48.7 \pm 7.7$ \\
\hline $\mathrm{G} 106.80+5.31(\mathrm{~b})$ & G106.800+5.314SMM & $22: 19: 18.9$ & $+63: 18: 59$ & $18.7 \pm 6.3$ & $4.7 \pm 0.6$ & $173.7 \pm 54.7$ & $9.3 \pm 1.1$ \\
\hline G109.87+2.11 & G109.871+2.113SMM & $22: 56: 18.3$ & $+62: 01: 44$ & $76.6 \pm 23.1$ & $14.5 \pm 1.5$ & $679.5 \pm 217.6$ & $75.7 \pm 9.9$ \\
\hline $\mathrm{G} 110.21+2.63$ & G110.211+2.609SMM & $22: 57: 07.3$ & $+62: 37: 22$ & $<5.4$ & $0.6 \pm 0.3$ & $<5.4$ & $7.1 \pm 2.5$ \\
\hline G111.28-0.66 (a) & $\mathrm{G}_{111.282-0.665 \mathrm{SMM}^{\mathrm{c}}}$ & $23: 16: 04.1$ & $+60: 01: 55$ & $22.0 \pm 6.7^{\dagger}$ & $2.4 \pm 0.3$ & $150.6 \pm 45.9$ & $13.1 \pm 2.2$ \\
\hline G111.28-0.66 (b) & G111.281-0.670SMM ${ }^{\mathrm{c}}$ & $23: 16: 04.4$ & $+60: 01: 38$ & & $1.5 \pm 0.2$ & & $5.8 \pm 0.6$ \\
\hline $\mathrm{G} 111.61+0.37$ & G111.612+0.374SMM & $23: 15: 31.3$ & $+61: 07: 12$ & $15.9 \pm 4.9$ & $2.9 \pm 0.3$ & $163.6 \pm 52.1$ & $14.9 \pm 3.2$ \\
\hline G133.95+1.06 & G133.949+1.064SMM & $02: 27: 04.7$ & $+61: 52: 23$ & $78.5 \pm 23.6$ & $20.4 \pm 2.1$ & $482.0 \pm 148.6$ & $62.1 \pm 8.7$ \\
\hline
\end{tabular}


M. A. Thompson et al.: A SCUBA imaging survey of UC HII regions, Online Material p 5

Table 2. continued.

\begin{tabular}{|c|c|c|c|c|c|c|c|}
\hline \multirow[t]{2}{*}{ UCHII field } & \multirow[t]{2}{*}{ Source } & \multirow{2}{*}{$\begin{array}{l}\text { RA (J2000) } \\
(\mathrm{h} \text { min s) }\end{array}$} & \multirow{2}{*}{$\begin{array}{l}\text { Dec (J2000) } \\
\left(0^{\circ}, \prime^{\prime \prime}\right)\end{array}$} & \multicolumn{2}{|c|}{ Peak flux (Jy/beam) } & \multicolumn{2}{|c|}{ Integrated flux density (Jy) } \\
\hline & & & & $450 \mu \mathrm{m}$ & $850 \mu \mathrm{m}$ & $450 \mu \mathrm{m}$ & $850 \mu \mathrm{m}$ \\
\hline G138.30+1.56 & G138.296+1.557SMM & $03: 01: 32.3$ & $+60: 29: 16$ & $35.2 \pm 10.7$ & $2.8 \pm 0.4$ & $183.1 \pm 57.0^{\dagger}$ & $19.6 \pm 6.9$ \\
\hline G188.79+1.03 & G188.795+1.031SMM & 06:09:07.2 & $+21: 50: 38$ & ․ & $2.1 \pm 0.4$ & . & $8.4 \pm 3.7$ \\
\hline G188.95+0.92 & G188.950+0.884SMM & 06:08:53.5 & $+21: 38: 13$ & $\ldots$ & $5.7 \pm 0.6$ & $\ldots$ & $24.4 \pm 6.8$ \\
\hline G213.88-11.84 & G213.880-11.836SMM & $06: 10: 50.8$ & $-06: 11: 48$ & $\ldots$ & $6.0 \pm 0.6$ & $\ldots$ & $31.8 \pm 3.9$ \\
\hline
\end{tabular}

(a) A noisy bolometer clipped from the final rebinned image is located close to this source and thus the measured flux is a lower limit to the true flux.

(b) These sources are separable in the unsmoothed $450 \mu \mathrm{m}$ image but blended in the $850 \mu \mathrm{m}$ image. The quoted $850 \mu \mathrm{m}$ flux is that of the blended single source. The coordinates quoted are measured from the $450 \mu \mathrm{m}$ image.

(c) These sources are separable in the $850 \mu \mathrm{m}$ images but blended into one source in the smoothed $450 \mu \mathrm{m}$ images. The quoted $450 \mu \mathrm{m}$ flux is the flux of the blended single source.

(d) A noisy bolometer clipped from the final rebinned $850 \mu \mathrm{m}$ image lies over this source and it was not possible to masure any sensible peak or integrated $850 \mu \mathrm{m}$ flux.

(e) This sources lies on the edge of the field of view and thus one or more of the quoted fluxes is a lower limit to the true flux. 
M. A. Thompson et al.: A SCUBA imaging survey of UC HII regions, Online Material p 6
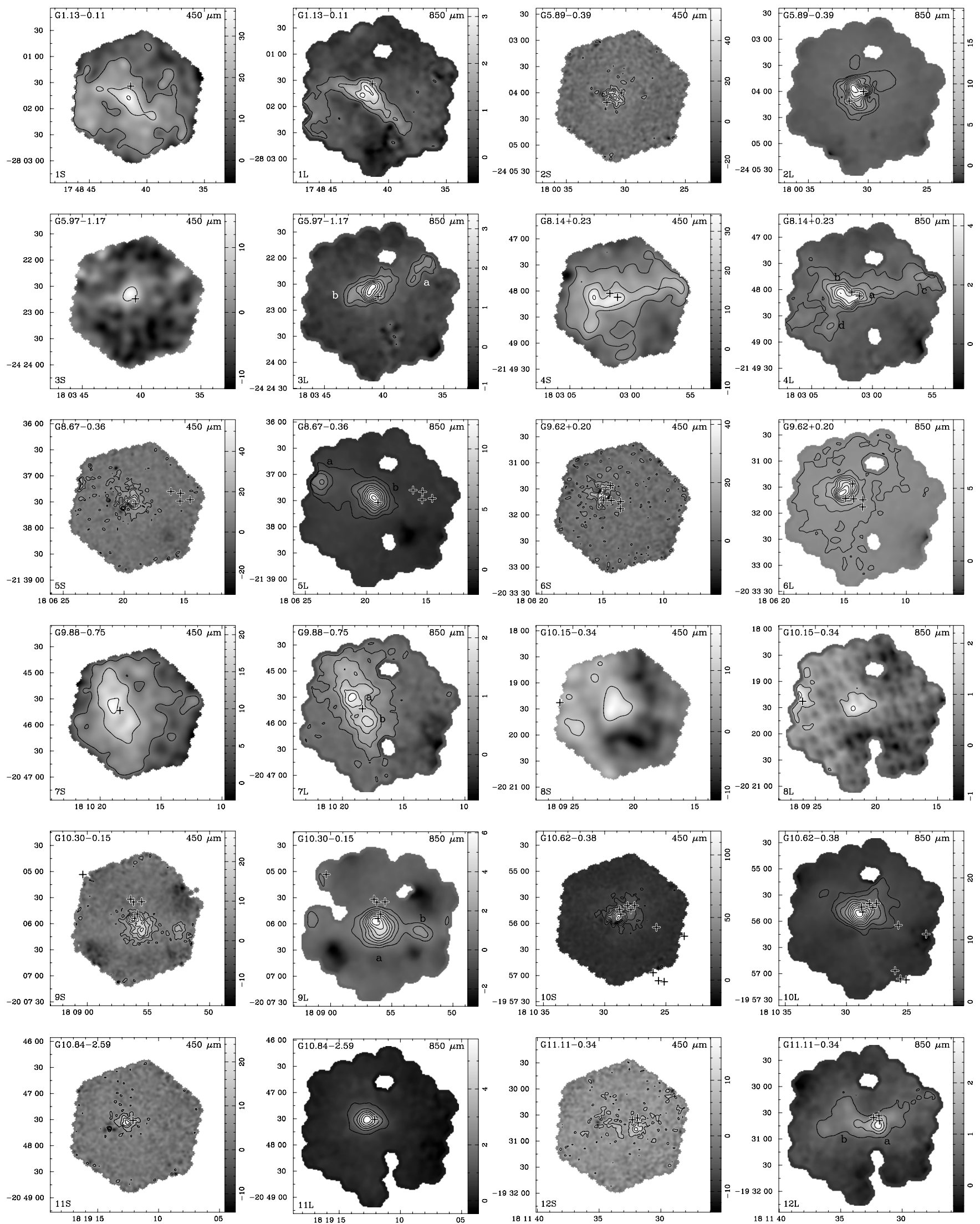

Fig. 1. SCUBA images from the survey with detections at both 450 and $850 \mu \mathrm{m}$. Each UC HII region is represented by a pair of images at $450 \mu \mathrm{m}$ (left image) and $850 \mu \mathrm{m}$ (right image). Coordinates are Right Ascension and Declination in the J2000 system. Crosses indicate the positions of ultracompact HII regions from Wood \& Churchwell (1989a), Kurtz et al. (1994), Becker et al. (1994) or Giveon et al. (2005). All images have been deconvolved with a model of the JCMT beam to remove the contribution from the error lobe and $450 \mu$ m images with limited signal-to-noise have been smoothed to the same resolution as the $850 \mu$ m images to improve the source detections. 
M. A. Thompson et al.: A SCUBA imaging survey of UC HII regions, Online Material $p 7$
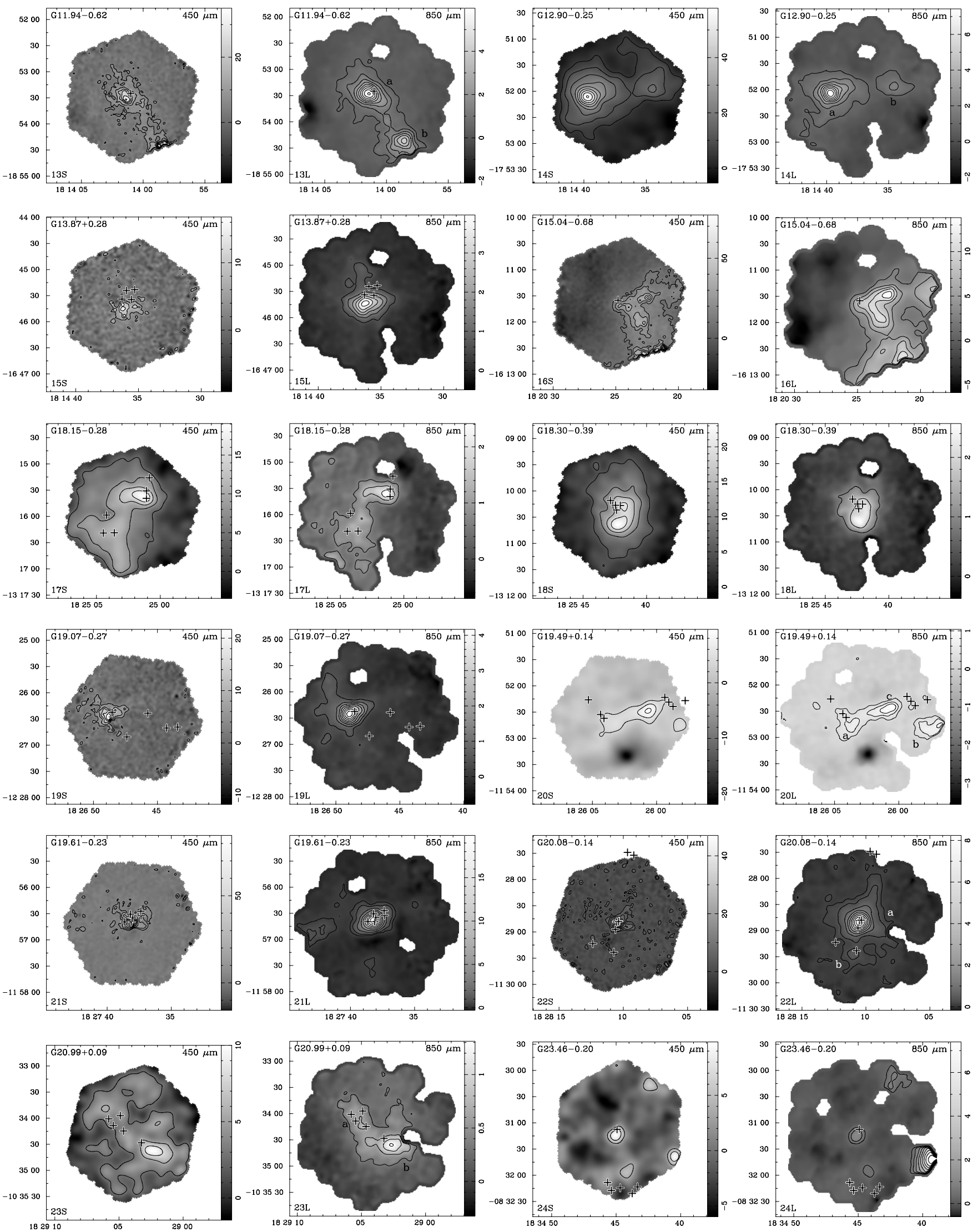

Fig. 1. continued. 
M. A. Thompson et al.: A SCUBA imaging survey of UC HII regions, Online Material $p 8$
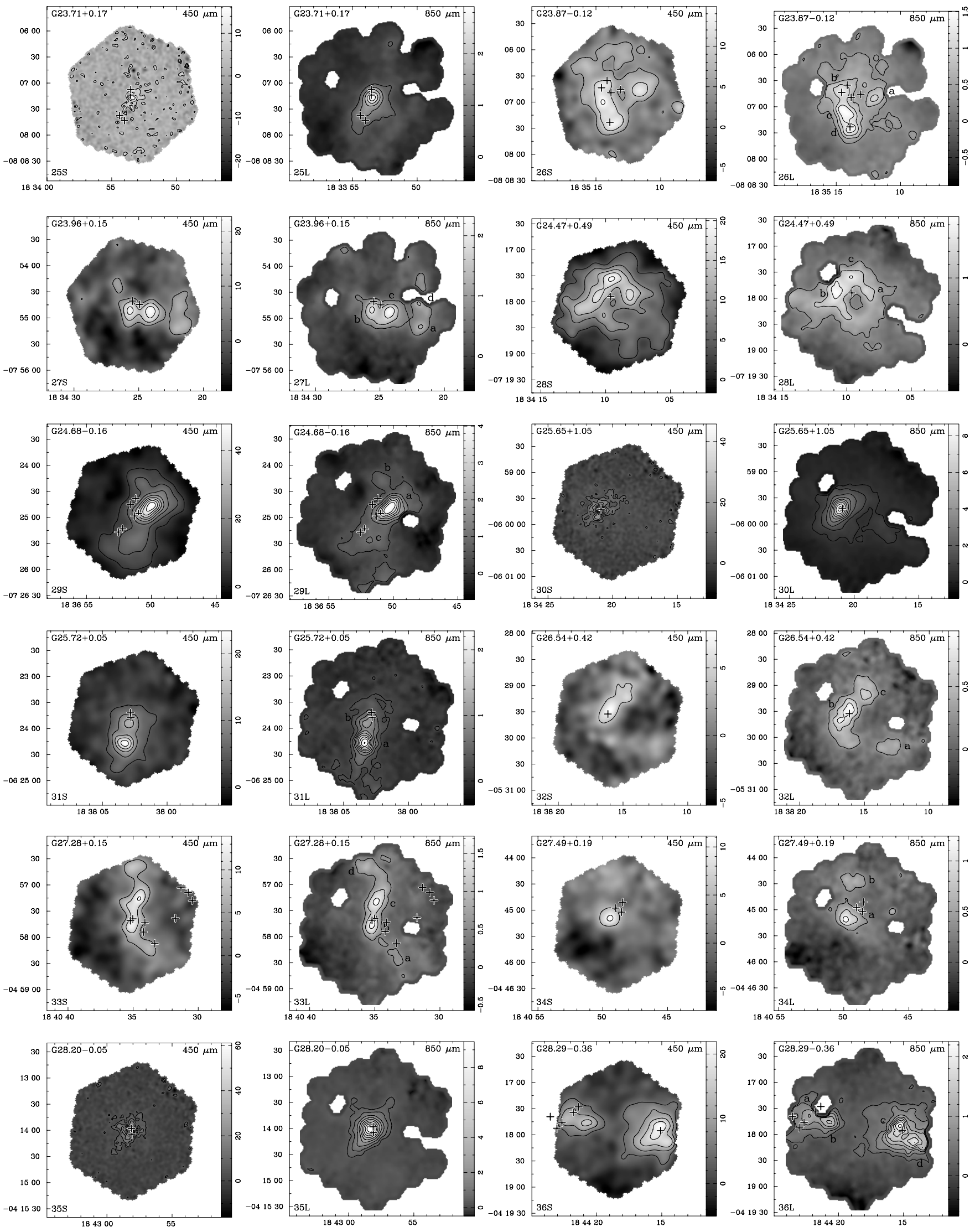

Fig. 1. continued. 
M. A. Thompson et al.: A SCUBA imaging survey of UC HII regions, Online Material $p 9$
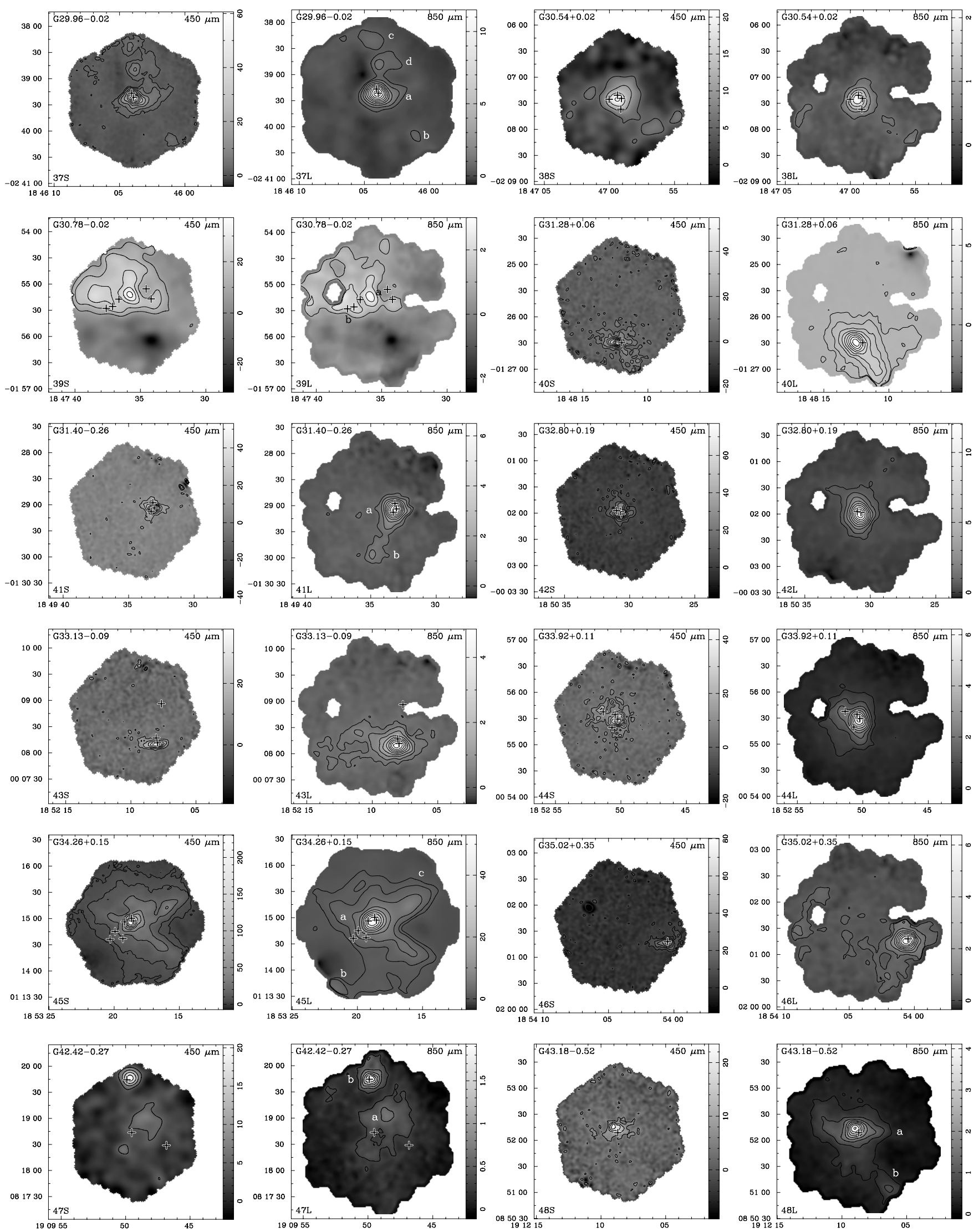

Fig. 1. continued. 
M. A. Thompson et al.: A SCUBA imaging survey of UC HII regions, Online Material $p 10$
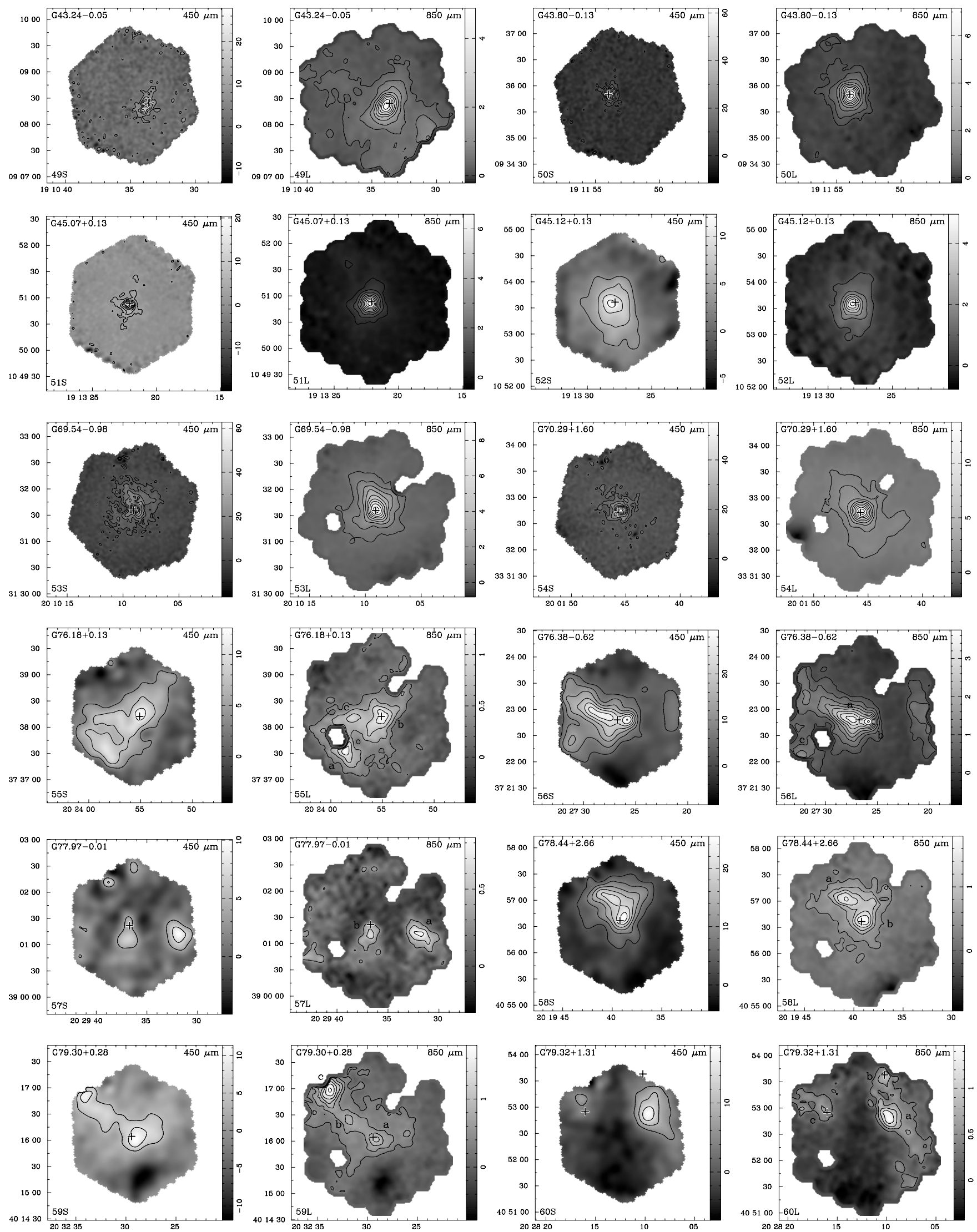

Fig. 1. continued. 
M. A. Thompson et al.: A SCUBA imaging survey of UC HII regions, Online Material $p 11$
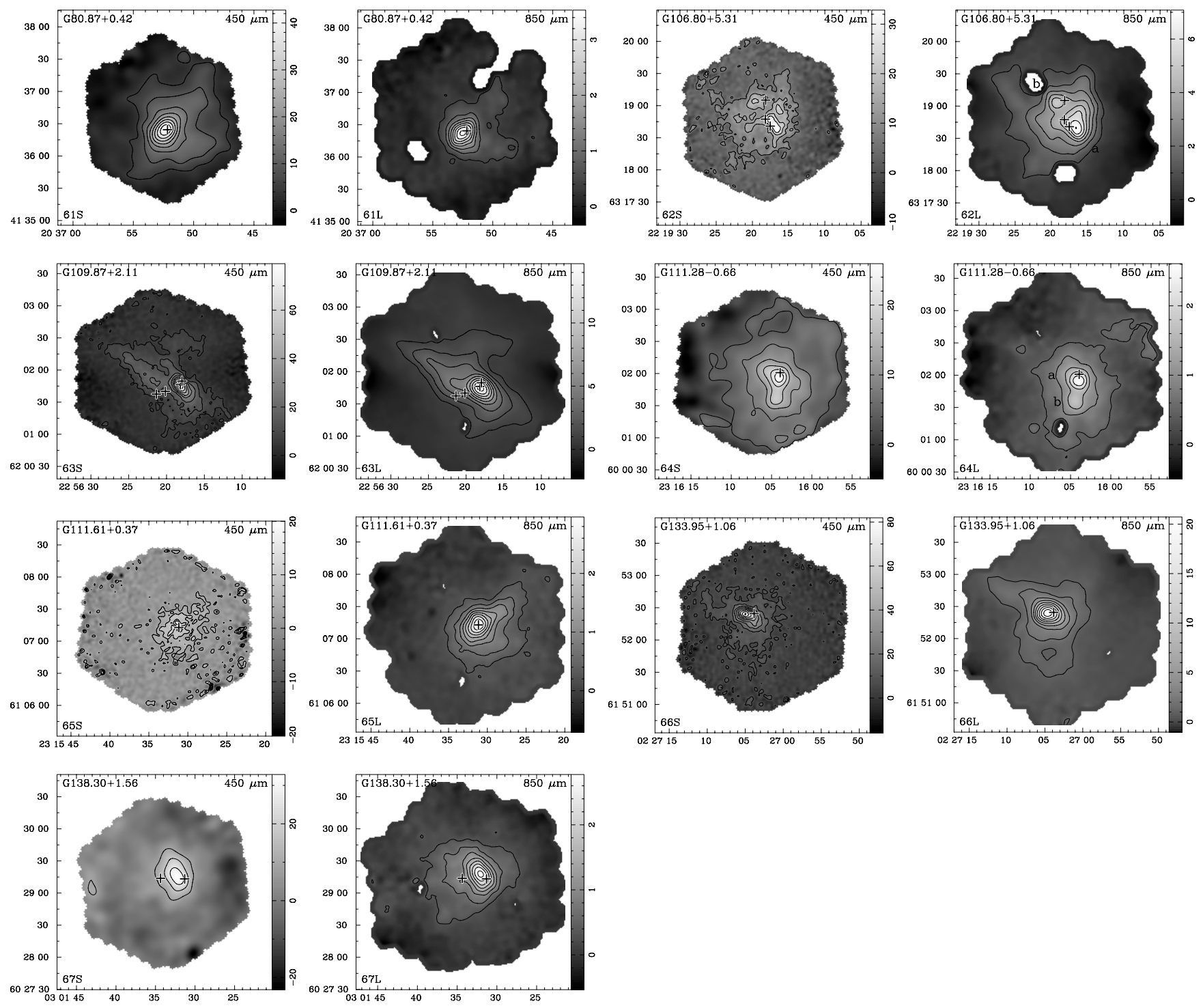

Fig. 1. continued. 
M. A. Thompson et al.: A SCUBA imaging survey of UC HII regions, Online Material $p 12$
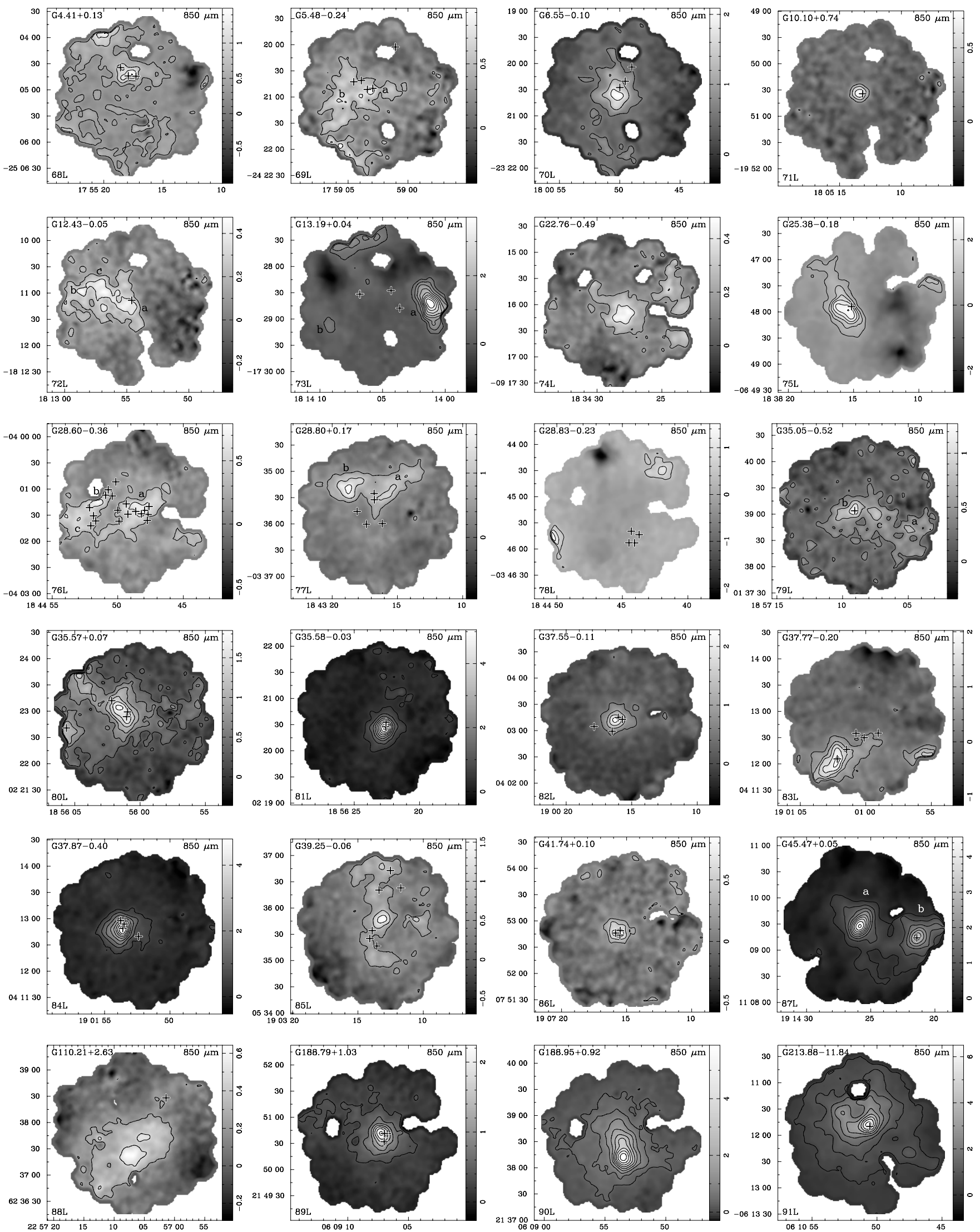

Fig. 2. SCUBA images of the ultracompact HII regions in the survey with detections at $850 \mu$ m only. Each UC HII region is represented by a pair of images at $450 \mu \mathrm{m}$ (left image) and $850 \mu \mathrm{m}$ (right image). Coordinates are Right Ascension and Declination in the J2000 system. Crosses indicate the positions of ultracompact HII regions from Wood \& Churchwell (1989a), Kurtz et al. (1994), Becker et al. (1994) or Giveon et al. (2005). All images have been deconvolved with a model of the JCMT beam to remove the contribution from the error lobe. 